\title{
Geology and geochemistry of the Baijiantan-Baikouquan ophiolitic mélanges: implications for geological evolution of west Junggar, Xinjiang, NW China
}

\author{
YONGFENG ZHU†, BO CHEN \& TIAN QIU
}

The Key Laboratory of Orogenic Belts and Crustal Evolution, Ministry of Education, Beijing China; School of Earth and Space Science, Peking University, Beijing 100871, China

(Received 22 August 2013; accepted 12 March 2014; first published online 23 April 2014)

\begin{abstract}
We report two newly identified Ordovician ophiolite belts in west Junggar, NW China: Tajin-Tarbahatai-Kujibai-Honguleleng (TTKH) and Tangbale-Baijiantan-Baikouquan (TBB) ophiolitic belts. These two ophiolitic belts provide constraints for the Palaeozoic reconstruction of Central Asia and the geological evolution of this region. The TTKH and TBB ophiolitic belts are dismembered parts of different ophiolitic belts which represent relics of Ordovician oceanic floor; they subducted to the north under the Chingiz-Tarbahatai arc and to the south under the Junggar plate, respectively. The Baijiantan-Baikouquan ophiolite mélanges comprise the major part of the TBB. Flat rare Earth element (REE) patterns with positive Eu anomalies and insignificant depletion of high-field-strength elements (HFSE) relative to melts of primitive mantle suggest a mid-ocean-ridge basalt (MORB) origin for the metagabbro. Lherzolite samples define a Sm-Nd isotopic isochron with age of $474 \mathrm{Ma}$ and $\varepsilon_{\mathrm{Nd}(t)}$ of +8 .9. Lherzolite samples with positive $\varepsilon_{\mathrm{Nd}(t)}$ values of +8.8 to +9.1 and initial ${ }^{87} \mathrm{Sr} /{ }^{86} \mathrm{Sr}$ ratios of 0.7037-0.7040 are rather homogeneous in $\mathrm{Sr}-\mathrm{Nd}$ isotopic composition, whereas metagabbro samples show wider $\mathrm{Sr}-\mathrm{Nd}$ isotopic compositional ranges with $\varepsilon_{\mathrm{Nd}(t)}$ of +5.9 to +11.0 . The $\mathrm{Sm}-\mathrm{Nd}$ isotopic isochron age (c. $380 \mathrm{Ma}$ ) for garnet amphibolite samples, consistent with a zircon $\mathrm{U}-\mathrm{Pb}$ age (c. $385 \mathrm{Ma}$ ) for metagabbro, represents a magmatic event prior to subduction. Thermodynamic calculations for garnet amphibolite yield a clockwise pressure-temperature path with peak metamorphic condition of $c$. $15 \mathrm{kbar}$ and $520-560^{\circ} \mathrm{C}$ at $342 \mathrm{Ma}$, indicating a subduction-channel setting. The $\mathrm{Rb}-\mathrm{Sr}$ isochron ages (335 Ma, $333 \mathrm{Ma}$ ) for metagabbro represent a metamorphic event during exhumation.
\end{abstract}

Keywords: ophiolitic mélange, metagabbro, garnet amphibolite, west Junggar.

\section{Introduction}

The core part of the Central Asian Orogenic Belt (CAOB, also called the Altaid Orogenic Belt) is a complex collage of island arcs, continental blocks and fragments of oceanic crust that amalgamated during Palaeozoic-Mesozoic time (Fig. 1). Several mutually contradictory plate tectonic interpretations have arisen since the 1990s (e.g. Coleman, 1989; Heinhorst et al. 2000; Zhang et al. 2007; Windley, Alexeiev \& Xiao, 2007; Xiao, Han \& Yuan, 2008; Zhu et al. 2009; Han et al. 2011; Wilhem, Windley \& Stamp, 2012). Zonenshain, Kuzmin \& Natapov (1990) suggested that the ophiolites of Central Asia represent the subducted crust of the Palaeo-Asian Ocean that separated east Europe, Siberia and Gondwana during the end of Neoproterozoic time. This interpretation was based on the assumption that most ophiolites were formed during late Precambrian-early Cambrian time and are therefore older than the spatially associated magmatic arcs. However, early Palaeozoic conodonts occur in most ophiolites of Kazakhstan, indicating a synchronicity of magmatic arcs and ophiolites and

$\dagger$ Author for correspondence: yfzhu@pku.edu.cn suggesting that the CAOB hosts relics of the former oceanic back-arc rather than truly oceanic basins (Yakubchuk, 2004). Sengor, Natal'in \& Burtman (1993) suggested that Precambrian crustal blocks and earlymiddle Palaeozoic turbiditic units could originally constitute the basement and accretionary wedges of only two magmatic arcs (Kipchak and Tuva-Mongol). This model implies that a subduction zone existed along the southern margin of the Siberian craton throughout $\mathrm{Pa}$ laeozoic time, producing a vast complex of arc and subduction-accretion material including scraped-off ophiolitic fragments at the front of seawards-migrating magmatic fronts. This would indicate a steady-state subduction-accretion over a prolonged period of time. In contrast, many other researchers (Zhang \& Huang, 1992; Mossakovsky et al. 1994; He et al. 2001; Khain, Bibikova \& Salnikova, 2003; Xu, He \& Li, 2006; Zhang et al. 2011; Yang et al. 2013; Zhu et al. 2013b) have identified distinct ophiolite belts in the core part of the $\mathrm{CAOB}$, interpreted as discrete suture zones between tectonic blocks. These authors are in favour of a model of punctuated accretion by collision and closure of multiple ocean basins now marked by ophiolitic sutures. 


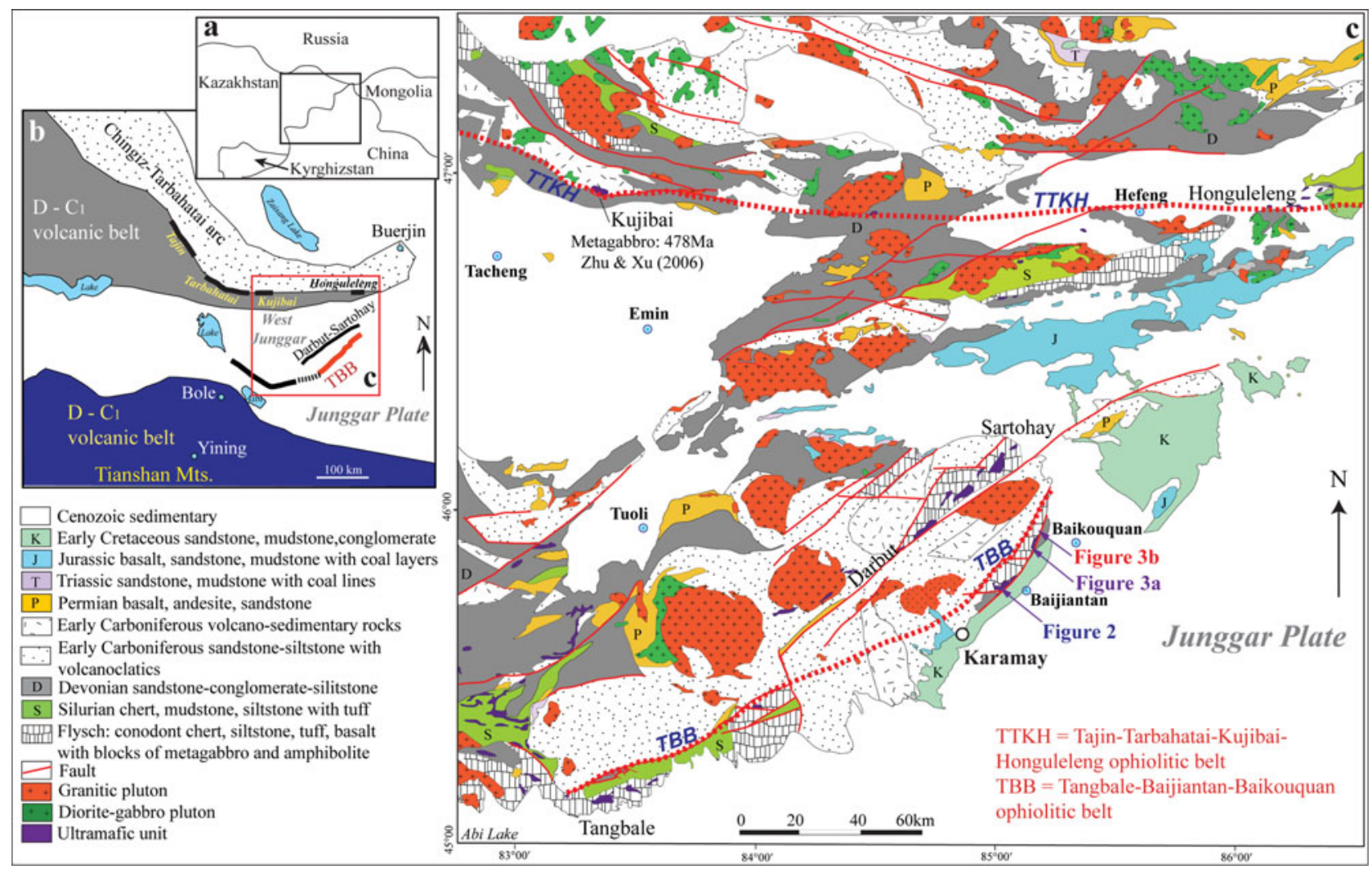

Figure 1. (a) Map showing the location of the studied area; (b) major tectonic units in the central part of CAOB; and (c) geological map of west Junggar (modified from Zhu et al. 2013b).

These controversial interpretations have encouraged the study of ophiolitic suites in the core part of the $\mathrm{CAOB}$ in order to provide new constraints on this issue. As a major component of the core part of the CAOB, west Junggar, located south of the Tajin-TarbahataiKujibai-Honguleleng ophiolitic belt (TTKH; Fig. 1b), is considered to be a Palaeozoic orogenic belt resulting from the convergence of the Siberian and KazakhstanJunggar plates (Kwon, Tilton \& Coleman, 1989; Zhu et al. 2013b). Several ophiolite mélanges occur in this region (Fig. 1c): the Tangbale ophiolite mélange to the SW; the Darbut-Sartohay ophiolite mélanges in the $\mathrm{NE}$; and the newly discovered Baijiantan-Baikouquan ophiolitic mélanges in the east. Blueschist in the Tangbale ophiolitic mélange was dated at $458-470 \mathrm{Ma}$ $\left({ }^{40} \mathrm{Ar} r{ }^{39} \mathrm{Ar}\right.$ on sodium amphibole; Zhang, 1997). The Darbut-Sartohay ophiolite belt was dated as being of Early Devonian age, based on radiolarian fossils in chert (Feng, 1986). Zircons separated from metagabbro in the Darbut-Sartohay ophiolite mélange were dated at $391 \mathrm{Ma}$ (Gu et al. 2009) and $426 \mathrm{Ma}$ (Chen \& Zhu, 2011).

This paper focuses on the petrology and geochemistry of the Baijiantan-Baikouquan ophiolite mélanges. We examine comprehensive geological, petrographic and geochemical datasets, discuss pressuretemperature $(P-T)$ conditions for metamorphic evolution of the ophiolitic mélanges based on thermodynamically calculated $P-T$ pseudosections for garnet amphibolite and interpret the lithological characteristics of west Junggar in a subduction- accretion scheme.

\section{Geology}

The original structures of ophiolitic mélanges in west Junggar have been deformed due to various late Palaeozoic geological events. Nevertheless, detailed field observations can be used to reconstruct the stratigraphic sections of most ophiolitic mélanges; we describe two distinct ophiolitic sequences. The Ordovician Tangbale ophiolitic mélange has a curved shape (Fig. 1c). The ophiolitic units are generally in contact with metamorphosed immature terrigenous sedimentary rocks of Silurian age along faults; these are locally uncomformably covered by early Silurian terrigenous detrital sedimentary rocks. Stratigraphic sections of ophiolites are reconstructed from base to top as follows: (1) ultramafic units consisting mainly of serpentinite with blocks of harzburgite, dunite and lherzolite, the main matrix of the ophiolitic mélange; (2) gabbro locally cross-cut by mafic dykes; (3) volcanic rocks consisting of altered basaltic lavas, pillow basalt, volcanic agglomerate and tuff; and (4) chert containing Middle Ordovician radiolaria grades upwards into turbidites at the top.

The Baijiantan-Baikouquan ophiolitic mélanges (Fig. 1c) consist of abyssal radiolarian chert interlayered with tuff, metabasaltic pillow lava with 


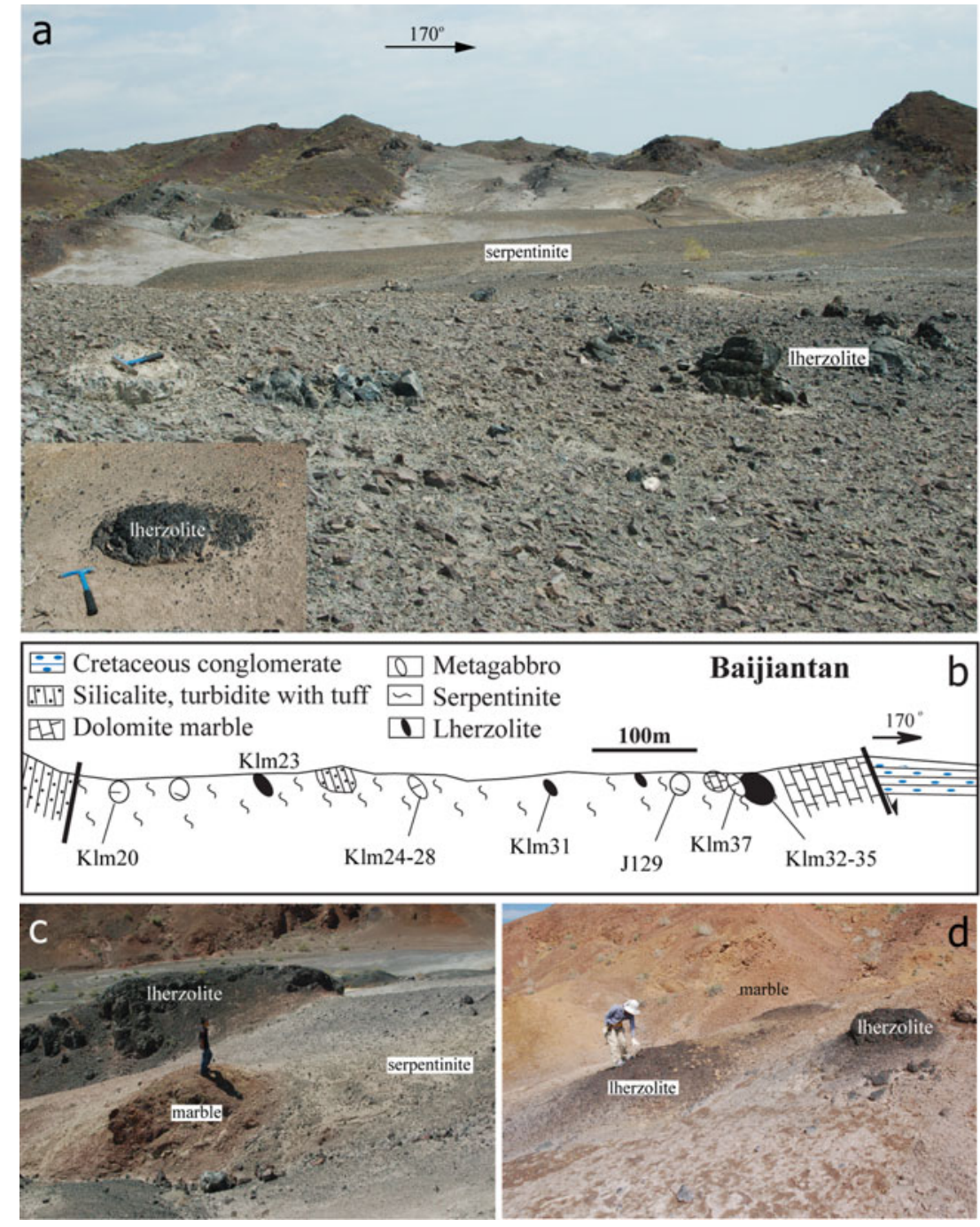

Figure 2. (a) Field view of the Baijiantan ophiolitic mélange showing lherzolite blocks; (b) geological section with sample locations of Baijiantan ophiolitic mélange; and (c, d) photographs showing lherzolite and marble blocks in serpentinite.

vesicular structure, metagabbro, spinel-bearing dolomite marble and serpentinized lherzolite and/or spinelbearing serpentinite. The ophiolite units have been strongly deformed and weathered (Fig. 2a). A geological section shows the relationship among different rock units across this ophiolitic mélange (Fig. 2b). Dolomite marble, present as bodies from a few decimetres to hundreds of metres in size, are intermingled with ultramafic units (Fig. 2c, d); ultramafic blocks occur in dolomite marble as relics (Fig. 2d) and one large dolomite marble lens marks the south boundary of this ophiolitic mélange. The abyssal radiolarian chert and tuffs are strongly deformed, with microfossils in the Baijiantan region indicating that the palaeo-ocean closed during the Late Ordovician period (He et al. 2007). The ophiolitic mélanges were covered by Devonian-lower Carboniferous volcanic-sedimentary rocks consisting mainly of sandstone, tuffaceous sandstone, siltstone, basalt, tuff and volcanic breccia (Zhu et al. 2011). The northern part of this ophiolitic mélange is covered by chert, siltstone and turbidite with tuff; metagabbro, marble and lherzolite lenses are randomly present in the serpentinite matrix.

The ophiolitic mélange in the Baikouquan region is restricted to a narrow zone bounded by faults. Strongly deformed chert and siltstone are present along the south boundary whereas the north boundary is a contact zone (Fig. 3a) between foliated basaltic rocks and early Carboniferous volcanic breccia, the latter unaffected by metamorphism or deformation. The central part of this ophiolitic mélange consists of serpentinite matrix with metagabbro and amphibolite lenses (Fig. 3b); most metagabbro lenses are located along the boundary between foliated basalt and serpentinite. Deformed basalt and chert cover the serpentinite.

Pre-Devonian pillow basalts found in the western mountains of Karamay city adjacent to the Baijiantan ophiolitic mélange preserve typical ocean-islandbasalt- (OIB-) like geochemical signatures (Zhu, Xu \& Wei, 2007); they are unlikely to be related to the ophiolites. These volcanic rocks differ from the Carboniferous rocks covering ophiolitic mélange in western 


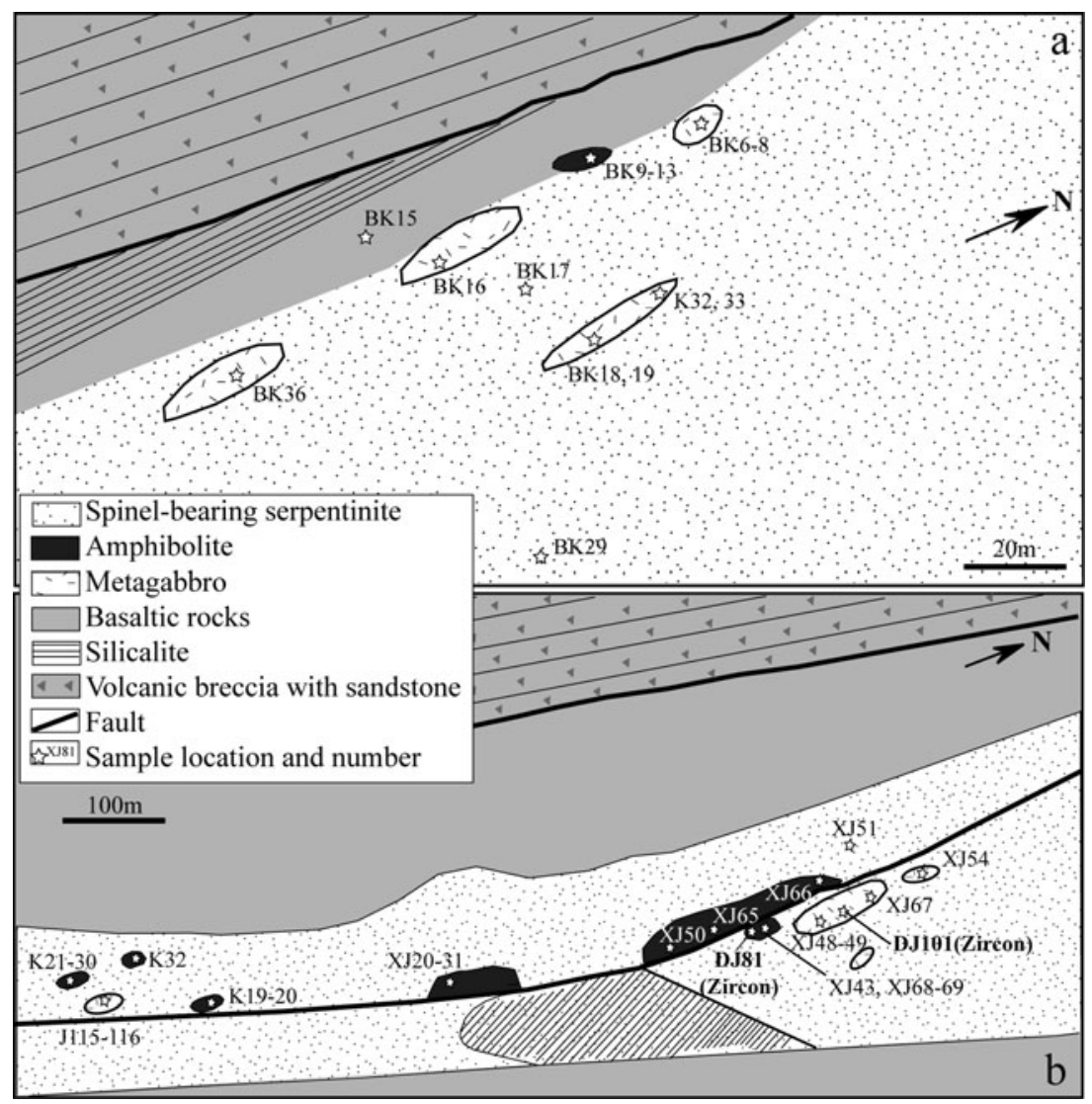

Figure 3. Geological maps showing two outcrops with sample locations in the Baikouquan region.

Junggar, which are also considered to be unrelated to the ophiolites (An \& Zhu, 2009; Zhu et al. 2013a).

\section{Analytical methods}

The compositions of mineral phases in polished thinsections were analysed with a Cameca SX100 electron probe microanalyser (EPMA) at the University of Stuttgart. This EPMA, equipped with five wavelengthdispersive spectrometers, was used to determine the contents of $\mathrm{Na}, \mathrm{Mg}, \mathrm{Al}, \mathrm{Si}, \mathrm{K}, \mathrm{Ca}, \mathrm{Ti}, \mathrm{Cr}, \mathrm{Mn}, \mathrm{Fe}$ and $\mathrm{Ba}$. Counting times were $20 \mathrm{~s}$ at the peak and on the background. We used synthetic and natural minerals, glasses (e.g. Ba glass for the $\mathrm{BaL} \alpha_{1}$-peak) and pure oxides as standards. An acceleration voltage and beam current of $15 \mathrm{kV}$ and $15 \mathrm{nA}$ were used; beam diameter was $c$. $5 \mu \mathrm{m}$ but also $1-2 \mu \mathrm{m}$ in the case of small mineral grains. The energy-dispersive system was used to identify minerals, and the compositions of representative major minerals are listed in the Supplementary Tables available at http://journals.cambridge.org/geo.

Zircons were hand-picked under a binocular microscope, and cathodoluminescence (CL) images obtained using a CAMECA SX-50 microprobe. Zircons were mounted in epoxy resin together with chips of the zircon standard TEMORA in the Beijing SHRIMP (sensitive high-resolution ion microprobe) Centre, Chinese Academy of Geological Sciences. The mount was ground down and polished so that the zircon interiors were exposed; zircon was photographed in reflected and transmitted light and under CL using a CAMECA SX-50 microprobe (accelerating voltage $10 \mathrm{kV}$, beam current $109 \mathrm{~mA}$ ). The mount was then cleaned and gold-coated. Isotopic analyses were performed with the SHRIMP II of the Beijing SHRIMP Centre (analytical procedures outlined by Williams, 1998). Prior to each analysis, the surface of the analysis site was pre-cleaned by rastering of the primary beam for 2 3 min to reduce or eliminate surface common $\mathrm{Pb}$. The reduced ${ }^{206} \mathrm{~Pb} /{ }^{238} \mathrm{U}$ ratios were normalized to 0.0668 , which is equivalent to the adopted age of $417 \mathrm{Ma}$ for zircon standard TEMORA. Six scans through the critical mass range were made to collect data, giving a slightly elliptical spot size of $c$. 25-30 $\mu \mathrm{m}$. Common$\mathrm{Pb}$ corrections were applied using the ${ }^{204} \mathrm{~Pb}$-correction method. Errors of individual analyses are given at the $1 \sigma$ level and are based on counting statistics; errors of pooled analyses are reported at the $2 \sigma$ confidence interval. The analytical data (Table 1) are graphically presented on conventional concordia diagrams.

Whole-rock samples were ground in an agate mill, after careful washing in distilled water and drying. Major elements were measured by an X-ray fluorescence spectrometer on glass disks made by fusion of whole-rock powder with lithium metaborate. Traceelement contents of whole-rock samples were analysed by quadrupole inductively coupled plasma mass spectrometry (ICP-MS); the precision is $<10 \%$ deviation from true values for most trace elements although it could be $>20 \%$ for elements with concentration less 
Table 1. SHRIMP data for zircons separated from Baikouquan metagabbro (DJ101) and amphibolite (DJ81).

\begin{tabular}{|c|c|c|c|c|c|c|c|c|c|c|c|c|c|c|c|c|c|c|}
\hline & $\%{ }^{206} \mathrm{~Pb}_{\mathrm{c}}$ & ${ }^{232} \mathrm{Th} /{ }^{238} \mathrm{U}$ & $\begin{array}{r}{ }^{206} \mathrm{~Pb} /{ }^{238} \mathrm{U} \\
\mathrm{Agge}(\mathrm{Ma})\end{array}$ & $\pm \mathrm{Ma}$ & $\begin{array}{c}\% \\
\text { Discordant }\end{array}$ & $\begin{array}{l}\text { Total } \\
{ }^{238} \mathrm{U} /{ }^{206} \mathrm{~Pb}\end{array}$ & $\pm \%$ & $\begin{array}{l}\text { Total } \\
{ }^{207} \mathrm{~Pb} /{ }^{206} \mathrm{~Pb}\end{array}$ & $\pm \%$ & ${ }^{238} \mathrm{U} / \mathrm{Pb}^{*}$ & $\pm \%$ & $\begin{array}{l}{ }^{207} \mathrm{~Pb}^{*} \\
{ }^{206} \mathrm{~Pb}^{*}\end{array}$ & $\pm \%$ & $\begin{array}{c}{ }^{207} \mathrm{~Pb}^{*} \\
{ }^{235} \mathrm{U}\end{array}$ & $\pm \%$ & $\begin{array}{c}{ }^{206} \mathrm{~Pb}^{*} \\
{ }^{238} \mathrm{U}\end{array}$ & $\pm \%$ & $\begin{array}{l}\text { Err } \\
\text { corr. }\end{array}$ \\
\hline \multicolumn{19}{|l|}{ DJ101 } \\
\hline-1.1 & 0.60 & 0.44 & 385 & 12 & -16 & 16.16 & 3.1 & 0.0579 & 2.3 & 16.26 & 3.1 & 0.0531 & 4.3 & 0.450 & 5.3 & 0.0615 & 3.1 & 0.581 \\
\hline-2.1 & 0.13 & 0.84 & 378.8 & 9.2 & 8 & 16.50 & 2.5 & 0.0560 & 1.7 & 16.52 & 2.5 & 0.0549 & 2.0 & 0.458 & 3.2 & 0.0605 & 2.5 & 0.78 \\
\hline-3.1 & 0.66 & 0.32 & 396 & 10 & 18 & 15.68 & 2.6 & 0.0621 & 5.1 & 15.79 & 2.6 & 0.0568 & 6.7 & 0.496 & 7.2 & 0.0633 & 2.6 & 0.365 \\
\hline-4.1 & 0.50 & 0.38 & 390.5 & 9.8 & 29 & 15.93 & 2.6 & 0.0626 & 4.2 & 16.01 & 2.6 & 0.0585 & 6.0 & 0.504 & 6.6 & 0.0624 & 2.6 & 0.395 \\
\hline-5.1 & 0.56 & 0.34 & 383.4 & 9.7 & -2 & 16.23 & 2.6 & 0.0586 & 2.5 & 16.32 & 2.6 & 0.0541 & 5.3 & 0.457 & 5.9 & 0.0613 & 2.6 & 0.443 \\
\hline-6.1 & 1.75 & 0.27 & 388 & 10 & 5 & 15.83 & 2.6 & 0.0690 & 4.6 & 16.11 & 2.8 & 0.0549 & 14 & 0.469 & 14 & 0.0621 & 2.8 & 0.196 \\
\hline-7.1 & 0.57 & 0.45 & 375.2 & 9.2 & 16 & 16.59 & 2.5 & 0.0604 & 2.0 & 16.69 & 2.5 & 0.0558 & 3.4 & 0.461 & 4.2 & 0.0599 & 2.5 & 0.596 \\
\hline-8.1 & 1.69 & 0.36 & 401 & 12 & -73 & 15.34 & 3.0 & 0.0645 & 3.9 & 15.60 & 3.0 & 0.0508 & 12 & 0.449 & 12 & 0.0641 & 3.0 & 0.25 \\
\hline-8.2 & 2.49 & 0.25 & 384 & 14 & 51 & 15.89 & 3.5 & 0.0853 & 6.6 & 16.30 & 3.7 & 0.0650 & 17 & 0.554 & 18 & 0.0614 & 3.7 & 0.208 \\
\hline-9.1 & 0.36 & 0.53 & 388.0 & 9.5 & -12 & 16.06 & 2.5 & 0.0563 & 2.0 & 16.12 & 2.5 & 0.0534 & 2.1 & 0.457 & 3.3 & 0.0620 & 2.5 & 0.765 \\
\hline-10.1 & 0.96 & 0.38 & 396 & 10 & -91 & 15.64 & 2.6 & 0.0581 & 6.7 & 15.79 & 2.6 & 0.0503 & 9.0 & 0.439 & 9.3 & 0.0633 & 2.6 & 0.281 \\
\hline-11.1 & 0.61 & 0.83 & 373.1 & 9.2 & -36 & 16.68 & 2.5 & 0.0567 & 3.8 & 16.78 & 2.5 & 0.0518 & 5.1 & 0.425 & 5.7 & 0.0596 & 2.5 & 0.442 \\
\hline-12.1 & 0.67 & 0.60 & 359.1 & 9.5 & -5 & 17.34 & 2.7 & 0.0587 & 2.2 & 17.46 & 2.7 & 0.0533 & 5.8 & 0.421 & 6.4 & 0.0573 & 2.7 & 0.421 \\
\hline-13.1 & 1.74 & 0.24 & 402 & 12 & 32 & 15.28 & 2.8 & 0.0736 & 6.1 & 15.55 & 3.0 & 0.0597 & 14 & 0.529 & 14 & 0.0643 & 3.0 & 0.213 \\
\hline $\begin{array}{l}-14.1 \\
\text { DJ81 }\end{array}$ & 1.72 & 0.41 & 388 & 11 & 43 & 15.85 & 2.9 & 0.0760 & 3.6 & 16.13 & 3.0 & 0.0622 & 9.1 & 0.532 & 9.6 & 0.0620 & 3.0 & 0.314 \\
\hline-1.1 & 1.41 & 0.49 & 364.2 & 7.7 & -16 & 16.96 & 2.1 & 0.0641 & 4.8 & 17.21 & 2.2 & 0.0527 & 8.9 & 0.422 & 9.2 & 0.0581 & 2.2 & 0.238 \\
\hline-2.1 & 2.82 & 0.42 & 378.6 & 9.7 & -71 & 16.06 & 2.6 & 0.0734 & 3.5 & 16.53 & 2.6 & 0.0506 & 11 & 0.422 & 11 & 0.0605 & 2.6 & 0.234 \\
\hline-3.1 & 3.36 & 0.35 & 359.6 & 8.6 & -68 & 16.85 & 2.3 & 0.0776 & 9.6 & 17.43 & 2.4 & 0.0500 & 21 & 0.399 & 21 & 0.0574 & 2.4 & 0.117 \\
\hline-4.1 & 3.75 & 0.47 & 369.7 & 8.4 & -75 & 16.31 & 2.2 & 0.0808 & 2.4 & 16.94 & 2.3 & 0.0503 & 13 & 0.410 & 13 & 0.0590 & 2.3 & 0.176 \\
\hline-5.1 & 1.48 & 0.37 & 348.0 & 7.9 & 43 & 17.76 & 2.2 & 0.0721 & 5.0 & 18.03 & 2.3 & 0.0602 & 12 & 0.461 & 12 & 0.0555 & 2.3 & 0.196 \\
\hline-6.1 & 1.85 & 0.28 & 352.1 & 9.3 & 16 & 17.48 & 2.7 & 0.0700 & 5.8 & 17.81 & 2.7 & 0.0552 & 11 & 0.427 & 11 & 0.0561 & 2.7 & 0.248 \\
\hline-7.1 & 1.08 & 0.38 & 357.8 & 7.0 & -10 & 17.33 & 2.0 & 0.0616 & 2.0 & 17.52 & 2.0 & 0.0529 & 4.8 & 0.416 & 5.2 & 0.0571 & 2.0 & 0.386 \\
\hline-8.1 & 1.52 & 0.37 & 359.8 & 7.3 & -54 & 17.15 & 2.1 & 0.0632 & 2.5 & 17.42 & 2.1 & 0.0509 & 6.8 & 0.403 & 7.1 & 0.0574 & 2.1 & 0.294 \\
\hline-9.1 & 1.03 & 0.40 & 364.4 & 8.7 & -53 & 17.02 & 2.4 & 0.0592 & 2.6 & 17.20 & 2.5 & 0.0509 & 5.7 & 0.408 & 6.2 & 0.0582 & 2.5 & 0.396 \\
\hline-10.1 & 2.91 & 0.35 & 370.6 & 8.6 & -8 & 16.41 & 2.3 & 0.0768 & 8.6 & 16.90 & 2.4 & 0.0533 & 17 & 0.435 & 17 & 0.0592 & 2.4 & 0.138 \\
\hline-11.1 & 2.62 & 0.44 & 352.0 & 13 & -86 & 17.37 & 3.7 & 0.0681 & 3.4 & 17.84 & 3.8 & 0.0468 & 15 & 0.361 & 16 & 0.0561 & $\begin{array}{l}2.4 \\
3.8\end{array}$ & 0.242 \\
\hline-12.1 & 0.16 & 0.54 & 362.2 & 6.9 & 2 & 17.27 & 2.0 & 0.0553 & 1.5 & 17.30 & 2.0 & 0.0540 & 2.2 & 0.430 & 2.9 & 0.0578 & 2.0 & 0.672 \\
\hline-13.1 & 0.55 & 0.47 & 360.0 & 6.9 & 14 & 17.31 & 2.0 & 0.0597 & 1.8 & 17.41 & 2.0 & 0.0552 & 2.8 & 0.437 & 3.4 & 0.0574 & 2.0 & 0.584 \\
\hline-14.1 & 1.08 & 0.62 & 367.5 & 7.3 & -31 & 16.86 & 2.0 & 0.0606 & 4.3 & 17.05 & 2.0 & 0.0519 & 6.7 & 0.420 & 7.0 & 0.0587 & 2.0 & 0.291 \\
\hline-15.1 & 1.19 & 0.74 & 356.6 & 6.9 & -26 & 17.37 & 2.0 & 0.0616 & 2.5 & 17.58 & 2.0 & 0.0519 & 4.6 & 0.407 & 5.0 & 0.0569 & 2.0 & 0.396 \\
\hline-16.1 & 1.69 & 0.39 & 366.3 & 7.6 & -34 & 16.82 & 2.1 & 0.0654 & 3.5 & 17.1 & 2.1 & 0.0517 & 7.5 & 0.417 & 7.8 & 0.0585 & 2.1 & 0.275 \\
\hline-17.1 & 1.07 & 0.91 & 341.9 & 7.2 & 0 & 18.16 & 2.1 & 0.0619 & 3.2 & 18.36 & 2.2 & 0.0533 & 8.0 & 0.401 & 8.3 & 0.0545 & 2.2 & 0.263 \\
\hline-18.1 & 4.64 & 0.36 & 356.5 & 8.4 & 22 & 16.77 & 2.2 & 0.0787 & 7.5 & 17.59 & 2.4 & 0.0410 & 25 & 0.320 & 25 & 0.0569 & 2.4 & 0.097 \\
\hline-19.1 & 2.00 & 0.32 & 364.8 & 7.8 & 76 & 16.83 & 2.1 & 0.0613 & 3.8 & 17.18 & 2.2 & 0.0450 & 11 & 0.361 & 12 & 0.0582 & 2.2 & 0.19 \\
\hline-20.1 & 0.28 & 0.64 & 359.5 & 6.8 & 3 & 17.39 & 1.9 & 0.0562 & 1.4 & 17.44 & 1.9 & 0.0540 & 2.0 & 0.427 & 2.8 & 0.0574 & 1.9 & 0.695 \\
\hline-21.1 & 1.36 & 0.49 & 366.2 & 7.8 & 16 & 16.88 & 2.2 & 0.0665 & 2.7 & 17.11 & 2.2 & 0.0555 & 5.7 & 0.448 & 6.1 & 0.0585 & 2.2 & 0.358 \\
\hline-22.1 & 0.64 & 0.55 & 358.3 & 6.9 & 5 & 17.39 & 2.0 & 0.0593 & 1.6 & 17.5 & 2.0 & 0.0542 & 3.3 & 0.427 & 3.8 & 0.0572 & 2.0 & 0.512 \\
\hline-23.1 & 0.58 & 0.29 & 368.7 & 7.4 & -28 & 16.89 & 2.0 & 0.0568 & 2.0 & 16.99 & 2.1 & 0.0521 & 3.2 & 0.423 & 3.8 & 0.0589 & 2.1 & 0.537 \\
\hline-24.1 & 4.72 & 0.34 & 360 & 11 & 82 & 16.58 & 2.6 & 0.0836 & 8.7 & 17.4 & 3.1 & 0.0450 & 33 & 0.360 & 33 & 0.0575 & 3.1 & 0.092 \\
\hline-25.1 & 7.15 & 0.25 & 355 & 14 & 25 & 16.39 & 3.5 & 0.1002 & 3.8 & 17.66 & 4.0 & 0.0420 & 40 & 0.330 & 40 & 0.0566 & 4.0 & 0.100 \\
\hline-26.1 & 2.19 & 0.61 & 374.8 & 9.5 & 7 & 16.34 & 2.3 & 0.0724 & 3.0 & 16.71 & 2.6 & 0.0548 & 18 & 0.452 & 18 & 0.0599 & 2.6 & 0.147 \\
\hline-27.1 & 3.26 & 0.40 & 381.7 & 9.6 & 34 & 15.86 & 2.4 & 0.0855 & 4.9 & 16.39 & 2.6 & 0.0594 & 14 & 0.499 & 14 & 0.0610 & 2.6 & 0.182 \\
\hline
\end{tabular}


than $1 \mathrm{ppm}$. The contents of rare Earth elements (REE) and other incompatible elements in clinopyroxene from metagabbro and garnet amphibolite were measured with laser ablation (LA) ICP-MS. The same method is used to measure the contents of trace elements in garnet from garnet amphibolite. Samples for isotopic analysis were dissolved in Teflon bombs after being spiked with ${ }^{84} \mathrm{Sr},{ }^{87} \mathrm{Rb},{ }^{150} \mathrm{Nd}$ and ${ }^{147} \mathrm{Sm}$ tracers prior to $\mathrm{HF}+\mathrm{HNO}_{3}$ (with a ratio of 2:1) dissolution. Strontium and neodymium were extracted by conventional ion exchange chromatographic techniques. Sr and Nd isotope ratios were measured using a Finnigan MAT 262 multiple collector thermal ionization mass spectrometer running in dynamic mode at the Institute of Geology and Geophysics in Beijing, according to the method described by Zhu et al. (2001). Replicate analyses of the $\mathrm{Sr}$ isotope reference material BCR-1 gave average ${ }^{87} \mathrm{Sr} /{ }^{86} \mathrm{Sr}$ values of $0.705086 \pm 0.000011(1 \sigma, n=$ 16 ; the recommended value for BCR-1 is $0.70501 \pm 8$; Balcaen et al. 2005). The ${ }^{87} \mathrm{Sr} /{ }^{86} \mathrm{Sr}$ ratio was corrected for instrumental mass fractionation assuming ${ }^{86} \mathrm{Sr} /{ }^{88} \mathrm{Sr}$ $=0.1194$; the ${ }^{143} \mathrm{Nd} /{ }^{144} \mathrm{Nd}$ ratio was corrected for instrumental mass fractionation assuming ${ }^{146} \mathrm{Nd} /{ }^{144} \mathrm{Nd}=$ 0.7219 . The Nd La Jolla reference material yielded an average ratio of ${ }^{143} \mathrm{Nd} /{ }^{144} \mathrm{Nd}=0.511842 \pm 0.000012$ $(1 \sigma, n=12$; recommended value for Nd La Jolla is 0.511849; Upadhyay, Scherer \& Mezger, 2008). Blanks were of the order $<0.3 \mathrm{ng}$ for $\mathrm{Sr}$ and $<0.1 \mathrm{ng}$ for $\mathrm{Nd}$. The Nd isotope data were normalized to the accepted reference values for La Jolla.

\section{Petrography}

\section{4.a. Lherzolite}

Lherzolite in the Baijiantan ophiolitic mélange consists mainly of olivine $(0-7 \%)$, orthopyroxene (20$33 \%$ ), clinopyroxene (30-45\%), spinel (2-4\%) and serpentine $(15-40 \%)$. Olivine and orthopyroxene were replaced by serpentine in most cases; olivine is locally present as inclusions in clinopyroxene or orthopyroxene (Fig. 4a). Brown spinel with homogeneous composition ( $\mathrm{Cr}$ number $8.5-12.9 \mathrm{~mol} . \%$ ) is replaced by ilmenite along rims and orthopyroxene is partly replaced by serpentine along rims and cleavages. Both clinopyroxene and orthopyroxene show exsolution textures; orthopyroxene lamellae occur in clinopyroxene (Fig. 4b, c) and parallel clinopyroxene lamellae occur in orthopyroxene (Fig. 4d). The compositional variation of these pyroxenes is shown in Supplementary Figure 1 and Supplementary Table 1 (both available at http://journals.cambridge.org/geo). Host clinopyroxene (diopside) contains enstatite-pigeonite lamellae and host orthopyroxene (enstatite) contains diopside lamellae. Diopside-enstatite pairs are common in the studied lherzolite samples and have very limited compositional variation in the En-Wo-Fs plots, whereas the trace element contents in these different pyroxene phases are distinguishable. For example, host clinopyroxene is rich in $\mathrm{Si}$ and poor in $\mathrm{Ti}$ and $\mathrm{Cr}$ relative to clinopyroxene lamellae, while host orthopyroxene and lamellae have a similar content of $\mathrm{Na}, \mathrm{Ti}$ and $\mathrm{Cr}$. The Ti mostly partitioned into diopside lamellae relative to their orthopyroxene host, and $\mathrm{Cr}$ partitioned largely into diopside lamellae during exsolution of orthopyroxene.

\section{4.b. Dolomite marble}

Dolomite marble lenses, ranging in size from several decimetres to hundreds of metres, occur in the Baijiantan ophiolitic mélange together with lherzolite lenses (Fig. 2b, c). Dolomite marble consists of dolomite $(20-45 \%)$, calcite $(10-15 \%)$, serpentine (10$15 \%)$, quartz (5-10\%), spinel (2-4\%) and magnetite $(2-5 \%)$. Zoned dolomite coexists with quartz and magnetite; quartz + magnetite + dolomite assemblage replaced serpentine in most cases. Brown spinel in marble was replaced by magnetite along its rim (Fig. $4 \mathrm{e})$. Dolomite marble lenses usually contain relics of spinel-bearing serpentinite, which suggest that the dolomite marble was transformed from spinel lherzolite (Zhu et al. 2008). The observed mineral assemblages and pyroxene pseudomorphs (Fig. 4f) resulted in the transformation of pyroxene into dolomite + quartz + magnetite via the reaction of pyroxene $+\mathrm{CO}_{2}=$ dolomite + magnetite + quartz; serpentine was also transformed to dolomite + quartz + magnetite via a reaction of serpentine + calcite $=$ dolomite + magnetite + quartz. Spinel is the only original mineral preserved during metamorphism, with a highly variable composition (Supplementary Table 2 and Supplementary Figure 2, available at http://journals.cambridge. org/geo). Spinel grains in dolomite marble are characterized by higher $\mathrm{Cr}$ number $(>0.6)$ and lower $\mathrm{Mg}$ number $(<0.6)$ than those in lherzolite.

\section{4.c. Metagabbro}

Metagabbro consists of clinopyroxene (25-40\%), plagioclase pseudomorphs (30-45\%), amphibole (5$10 \%$ ) and other second mineral phases (zoisite, albite, chlorite, ilmenite and quartz); plagioclase pseudomorphs consist mainly of zoisite and albite (Fig. 5ad). As a mineral phase crystallized from magma, clinopyroxene was replaced by amphibole (Fig. 5e, f) and garnet occurs along the rim of plagioclase pseudomorphs (Fig. 5g, h). The latter suggests a transformation from plagioclase to garnet, which was accompanied by growth of chlorite and ilmenite (Fig. 5h).

\section{4.d. Garnet amphibolite}

Garnet amphibolite blocks, ranging in size from a few decimetres to several metres, occur rarely in the Baikouquan region. Garnet amphibolite contains various amounts of garnet and amphibole with minor amounts of zoisite, epidote, chlorite, clinopyroxene, ilmenite, biotite and sphene. Garnet grains vary greatly in size (from $<0.1 \mathrm{~mm}$ to $>1 \mathrm{~mm}$ ), and large garnet 
Table 2. Trace element concentrations of clinopyroxene in metagabbro by LA-ICP-MS (in ppm).

\begin{tabular}{|c|c|c|c|c|c|c|c|c|c|c|c|c|c|c|c|c|c|c|c|c|}
\hline Spot & 1 & 2 & 3 & 4 & 5 & 6 & 7 & 8 & 9 & 10 & 11 & 12 & 13 & 14 & 15 & 16 & 17 & 18 & 19 & 20 \\
\hline $\mathrm{Sc}$ & 100.4 & 80.77 & 82.23 & 85.5 & 91.32 & 96.45 & 76.63 & 83.05 & 88.80 & 76.26 & 81.54 & 105.7 & 90.70 & 82.39 & 83.22 & 81.14 & 69.18 & 88.54 & 82.75 & 78.31 \\
\hline V & 328.4 & 219.1 & 244.1 & 270.6 & 292.0 & 328.0 & 172.5 & 239.8 & 248.2 & 150.7 & 224.8 & 391.0 & 296.8 & 274.0 & 285.9 & 276.7 & 200.0 & 259.0 & 277.0 & 242.5 \\
\hline $\mathrm{Cr}$ & 546.1 & 516.1 & 496.2 & 582.2 & 504.4 & 809.4 & 353.8 & 495.9 & 631.3 & 348.4 & 489.9 & 972.0 & 224.5 & 373.8 & 487.0 & 575.2 & 711.0 & 620.2 & 725.4 & 501.9 \\
\hline Co & 38.33 & 39.04 & 45.19 & 42.22 & 39.23 & 41.23 & 37.13 & 42.43 & 36.72 & 38.08 & 34.19 & 50.94 & 41.88 & 41.22 & 38.26 & 37.06 & 39.04 & 35.79 & 43.88 & 40.18 \\
\hline $\mathrm{Ni}$ & 72.99 & 77.28 & 98.46 & 91.81 & 79.04 & 90.70 & 84.32 & 114.2 & 77.13 & 100.4 & 64.85 & 129.9 & 80.37 & 82.35 & 78.59 & 77.59 & 97.07 & 69.99 & 103.2 & 86.85 \\
\hline $\mathrm{Ga}$ & 4.09 & 2.74 & 4.63 & 3.90 & 3.77 & 4.30 & 3.21 & 3.62 & 4.17 & 2.19 & 3.80 & 7.47 & 4.59 & 3.95 & 3.82 & 3.97 & 3.44 & 3.41 & 4.59 & 3.96 \\
\hline Cs & 0.041 & 0.009 & 0.042 & 0.010 & 0.010 & 0.009 & 0.233 & 0.056 & 0.081 & 0.009 & 0.144 & 0.070 & 0.025 & 0.028 & 0.035 & 0.011 & 0.033 & 0.013 & 0.036 & 0.008 \\
\hline $\mathrm{Rb}$ & 0.13 & 0.018 & 0.12 & 0.17 & 0.065 & 0.16 & 0.12 & 0.21 & 0.29 & 0.051 & 0.084 & 0.54 & 0.24 & 0.18 & 0.18 & 0.093 & 0.083 & 0.048 & 0.27 & 0.14 \\
\hline $\mathrm{Ba}$ & 2.37 & 0.36 & 0.90 & 0.83 & 0.24 & 0.50 & 0.47 & 1.30 & 0.97 & 0.74 & 0.43 & 1.02 & 0.61 & 0.55 & 2.12 & 0.85 & 0.81 & 0.69 & 1.89 & 1.08 \\
\hline Th & 0.010 & 0.007 & 0.007 & 0.007 & 0.007 & 0.006 & 0.008 & 0.023 & 0.018 & 0.007 & 0.006 & 0.009 & 0.012 & 0.013 & 0.005 & 0.007 & 0.007 & 0.003 & 0.017 & 0.009 \\
\hline $\mathrm{U}$ & 0.007 & 0.006 & 0.009 & 0.009 & 0.005 & 0.007 & 0.005 & 0.017 & 0.022 & 0.005 & 0.009 & 0.012 & 0.012 & 0.008 & 0.008 & 0.008 & 0.007 & 0.005 & 0.011 & 0.007 \\
\hline $\mathrm{Ta}$ & 0.002 & 0.004 & 0.005 & 0.011 & 0.004 & 0.008 & 0.005 & 0.005 & 0.005 & 0.004 & 0.002 & 0.010 & 0.005 & 0.003 & 0.004 & 0.006 & 0.003 & 0.002 & 0.004 & 0.006 \\
\hline $\mathrm{Nb}$ & 0.015 & 0.006 & 0.034 & 0.123 & 0.050 & 0.114 & 0.017 & 0.050 & 0.023 & 0.007 & 0.008 & 0.119 & 0.048 & 0.027 & 0.049 & 0.065 & 0.009 & 0.015 & 0.073 & 0.070 \\
\hline $\mathrm{La}$ & 0.18 & 0.16 & 0.15 & 0.20 & 0.20 & 0.22 & 0.15 & 0.18 & 0.16 & 0.14 & 0.14 & 0.31 & 0.23 & 0.15 & 0.17 & 0.17 & 0.13 & 0.12 & 0.18 & 0.18 \\
\hline $\mathrm{Ce}$ & 0.97 & 0.84 & 0.77 & 0.98 & 1.00 & 1.13 & 0.66 & 0.91 & 0.80 & 0.63 & 0.70 & 1.47 & 1.12 & 0.76 & 0.88 & 0.91 & 0.63 & 0.70 & 0.97 & 0.89 \\
\hline $\mathrm{Pb}$ & 0.088 & 0.019 & 0.075 & 0.026 & 0.024 & 0.037 & 0.15 & 0.090 & 0.16 & 0.088 & 0.090 & 0.12 & 0.024 & 0.029 & 0.054 & 0.025 & 0.050 & 0.057 & 0.065 & 0.032 \\
\hline $\mathrm{Sr}$ & 6.61 & 4.96 & 6.11 & 6.85 & 5.45 & 6.59 & 7.92 & 6.54 & 8.83 & 5.03 & 7.34 & 12.73 & 8.34 & 8.10 & 11.65 & 5.82 & 4.74 & 4.78 & 7.78 & 6.54 \\
\hline $\mathrm{Nd}$ & 1.66 & 1.36 & 1.35 & 1.53 & 1.75 & 1.88 & 1.09 & 1.45 & 1.34 & 0.91 & 1.19 & 2.49 & 1.69 & 1.34 & 1.50 & 1.45 & 1.04 & 1.33 & 1.61 & 1.54 \\
\hline $\mathrm{Zr}$ & 13.36 & 8.91 & 10.35 & 11.08 & 17.91 & 13.60 & 7.75 & 9.37 & 10.62 & 9.14 & 6.66 & 14.95 & 11.17 & 5.70 & 10.37 & 9.30 & 3.79 & 8.28 & 9.42 & 8.79 \\
\hline $\mathrm{Hf}$ & 0.40 & 0.28 & 0.27 & 0.38 & 0.76 & 0.54 & 0.36 & 0.31 & 0.42 & 0.42 & 0.26 & 0.62 & 0.39 & 0.21 & 0.46 & 0.43 & 0.11 & 0.34 & 0.37 & 0.38 \\
\hline $\mathrm{Ti}$ & 2129 & 1233 & 1591 & 2115 & 2238 & 2798 & 855 & 1907 & 1789 & 661 & 1254 & 3740 & 2213 & 1682 & 2175 & 2070 & 967 & 1851 & 2310 & 1799 \\
\hline $\mathrm{Sm}$ & 0.89 & 0.66 & 0.67 & 0.72 & 0.93 & 1.00 & 0.51 & 0.77 & 0.66 & 0.47 & 0.58 & 1.31 & 0.88 & 0.66 & 0.86 & 0.69 & 0.42 & 0.61 & 0.83 & 0.76 \\
\hline $\mathrm{Eu}$ & 0.31 & 0.25 & 0.25 & 0.31 & 0.31 & 0.37 & 0.18 & 0.28 & 0.24 & 0.17 & 0.24 & 0.50 & 0.34 & 0.28 & 0.30 & 0.28 & 0.19 & 0.27 & 0.34 & 0.31 \\
\hline $\mathrm{Y}$ & 10.05 & 7.83 & 7.52 & 8.79 & 10.11 & 11.04 & 5.25 & 7.46 & 6.91 & 4.67 & 6.37 & 12.91 & 9.46 & 7.07 & 7.77 & 7.46 & 4.92 & 6.94 & 8.21 & 7.32 \\
\hline $\mathrm{Lu}$ & 0.17 & 0.13 & 0.13 & 0.15 & 0.16 & 0.17 & 0.093 & 0.13 & 0.12 & 0.080 & 0.12 & 0.22 & 0.16 & 0.13 & 0.14 & 0.12 & 0.095 & 0.13 & 0.16 & 0.12 \\
\hline $\mathrm{Pr}$ & 0.23 & 0.20 & 0.18 & 0.22 & 0.24 & 0.24 & 0.15 & 0.22 & 0.19 & 0.13 & 0.16 & 0.32 & 0.25 & 0.18 & 0.21 & 0.20 & 0.14 & 0.16 & 0.21 & 0.21 \\
\hline $\mathrm{Gd}$ & 1.32 & 1.06 & 1.01 & 1.13 & 1.41 & 1.61 & 0.76 & 1.06 & 0.95 & 0.67 & 0.90 & 1.93 & 1.29 & 1.02 & 1.11 & 1.07 & 0.687 & 1.0 & 1.18 & 1.05 \\
\hline $\mathrm{Tb}$ & 0.29 & 0.21 & 0.22 & 0.23 & 0.31 & 0.31 & 0.17 & 0.22 & 0.22 & 0.14 & 0.19 & 0.39 & 0.28 & 0.22 & 0.24 & 0.24 & 0.15 & 0.21 & 0.25 & 0.227 \\
\hline Dy & 1.93 & 1.44 & 1.46 & 1.63 & 1.98 & 2.12 & 1.12 & 1.45 & 1.43 & 0.893 & 1.32 & 2.60 & 1.88 & 1.41 & 1.63 & 1.61 & 1.02 & 1.45 & 1.69 & 1.51 \\
\hline Но & 0.46 & 0.34 & 0.32 & 0.37 & 0.44 & 0.46 & 0.24 & 0.34 & 0.32 & 0.21 & 0.30 & 0.58 & 0.42 & 0.33 & 0.37 & 0.33 & 0.22 & 0.32 & 0.38 & 0.34 \\
\hline Er & 1.13 & 0.88 & 0.91 & 1.02 & 1.22 & 1.27 & 0.63 & 0.92 & 0.83 & 0.56 & 0.78 & 1.5 & 1.14 & 0.90 & 0.97 & 0.91 & 0.63 & 0.84 & 1.04 & 0.88 \\
\hline $\mathrm{Tm}$ & 0.15 & 0.13 & 0.13 & 0.14 & 0.17 & 0.17 & 0.098 & 0.14 & 0.12 & 0.087 & 0.12 & 0.2 & 0.17 & 0.14 & 0.15 & 0.14 & 0.093 & 0.12 & 0.15 & 0.13 \\
\hline $\mathrm{Yb}$ & 1.12 & 0.90 & 0.90 & 0.97 & 1.08 & 1.27 & 0.59 & 0.85 & 0.81 & 0.55 & 0.79 & 1.5 & 1.09 & 0.91 & 0.98 & 0.97 & 0.65 & 0.83 & 1.05 & 0.93 \\
\hline$(\mathrm{Ce} / \mathrm{Yb})_{\mathrm{N}}$ & 0.24 & 0.26 & 0.24 & 0.28 & 0.26 & 0.25 & 0.31 & 0.30 & 0.27 & 0.32 & 0.25 & 0.27 & 0.28 & 0.23 & 0.25 & 0.26 & 0.27 & 0.23 & 0.26 & 0.26 \\
\hline$(\mathrm{Gd} / \mathrm{Yb})_{\mathrm{N}}$ & 0.98 & 0.98 & 0.93 & 0.96 & 1.08 & 1.05 & 1.07 & 1.02 & 0.97 & 1.01 & 0.94 & 1.0 & 0.98 & 0.93 & 0.94 & 0.92 & 0.88 & 1.07 & 0.93 & 0.93 \\
\hline $\mathrm{Eu} / \mathrm{Eu} *$ & 0.88 & 0.90 & 0.93 & 1.04 & 0.82 & 0.89 & 0.87 & 0.95 & 0.93 & 0.90 & 1.03 & 0.95 & 0.98 & 1.05 & 0.94 & 1.00 & 1.07 & 1.01 & 1.04 & 1.06 \\
\hline
\end{tabular}



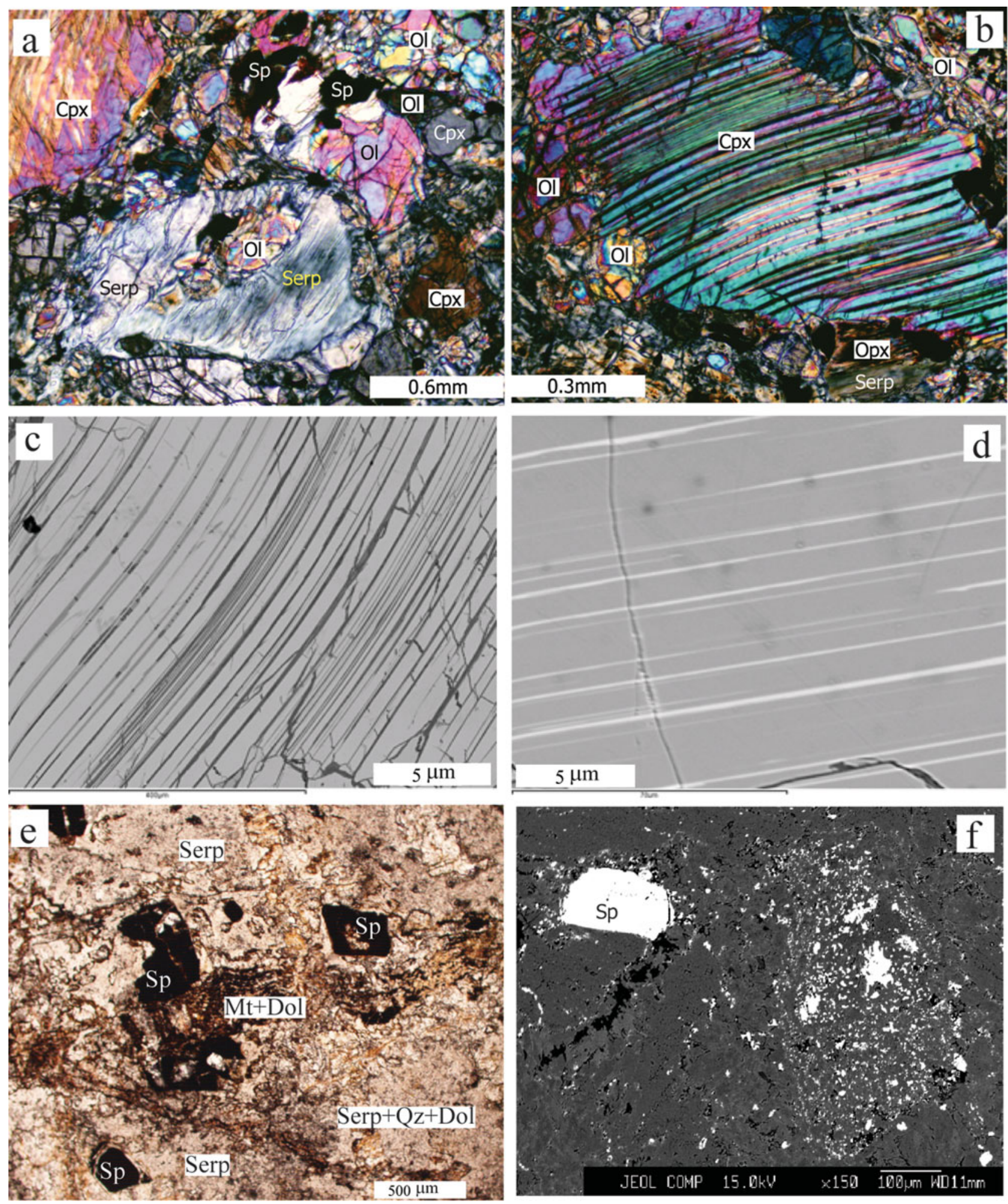

Figure 4. Photomicrographs showing (a) mineral phases in lherzolite, cross-polarized light; (b) clinopyroxene with exsolution texture; ductile deformation in lherzolite, cross-polarized light; (c) orthopyroxene lamellae exsolved from host clinopyroxene, BSE; (d) clinopyroxene lamellae exsolved from host orthopyroxene, BSE; (e) spinel and serpentine relics in dolomite marble, plane-polarized light; and (f) BSE image showing orthopyroxene pseudomorph in dolomite marble. Cpx - clinopyroxene; Dol - dolomite; Mt magnetite; Ol - olivine; Opx - orthopyroxene; Qz - quartz; Serp - serpentine; Sp - spinel.

grains are usually cracked (Figs 6a, 7a). Zoisite occurs in the matrix, pseudomorphing plagioclase (Fig. 6a), and fills cracks in garnet and clinopyroxene grains, the latter containing ilmenite inclusions (Fig. 6b). Garnet contains various kinds of mineral inclusions includ- ing clinopyroxene, rutile, apatite, ilmenite, biotite and quartz (Fig. 7b, g).

Garnet compositions (Supplementary Table 3, available at http://journals.cambridge.org/geo) vary significantly from one sample to another (Fig. 8), 

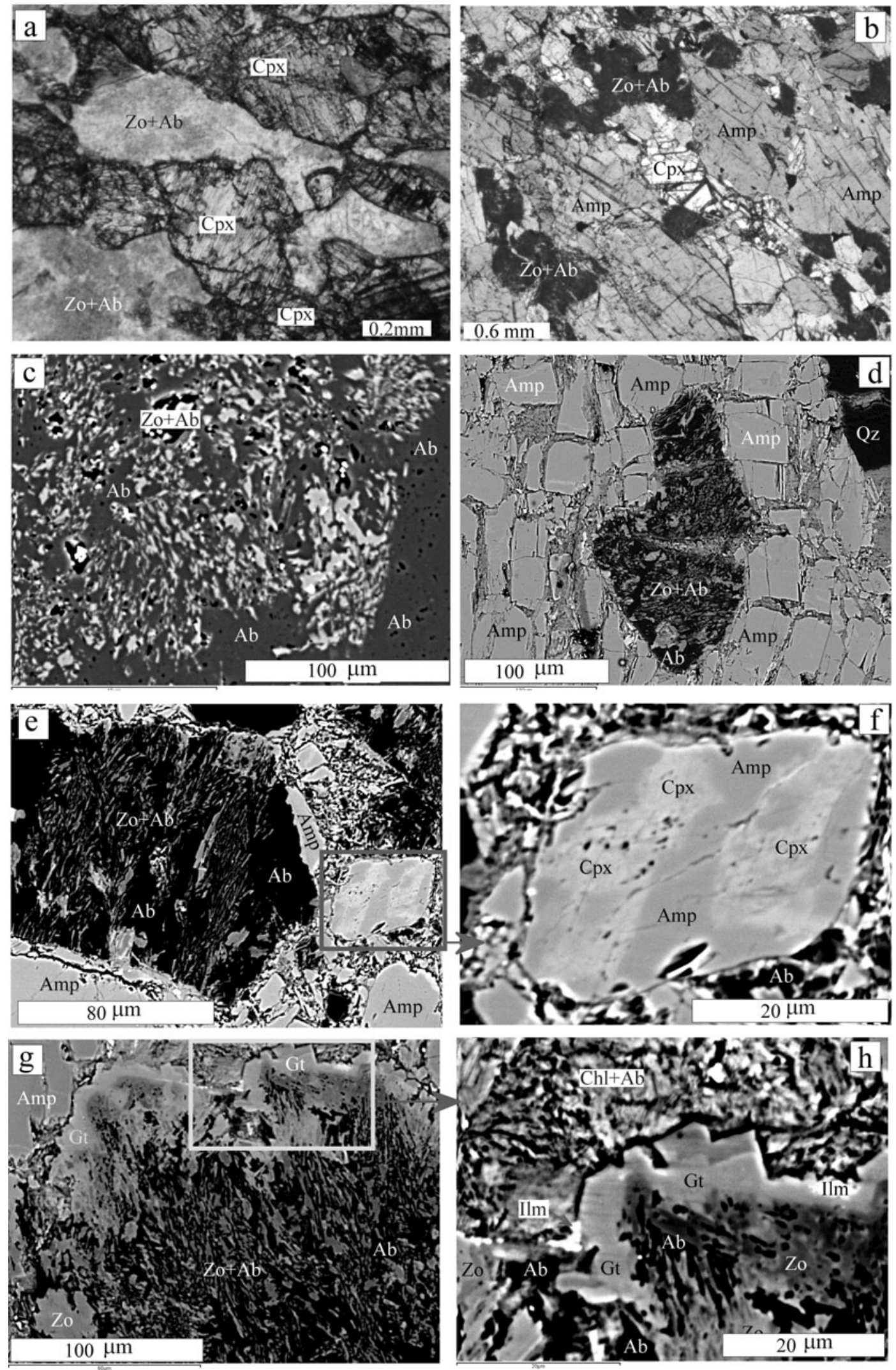

Figure 5. Photomicrograph showing mineral relationship in metagabbro: (a) metagabbro consisting of clinopyroxene and pseudomorph plagioclase $(\mathrm{Zo}+\mathrm{Ab})$; plane-polarized light; (b) metagabbro consisting of pseudomorph plagioclase, amphibole and clinopyroxene; plane-polarized light; (c) BSE image showing pseudomorph plagioclase consisting of zoisite and albite; (d) BSE image showing pseudomorph plagioclase coexisting with amphibole; (e, f) BSE images showing a pseudomorph plagioclase and amphibole with clinopyroxene relics; $(\mathrm{g}, \mathrm{h})$ garnet growth along rim of pseudomorph plagioclase, BSE. Ab - albite; Amp - amphibole; Chl - chlorite; $\mathrm{Cpx}$ - clinopyroxene; Gt - garnet; Ilm - ilmenite; Zo - zoisite. 


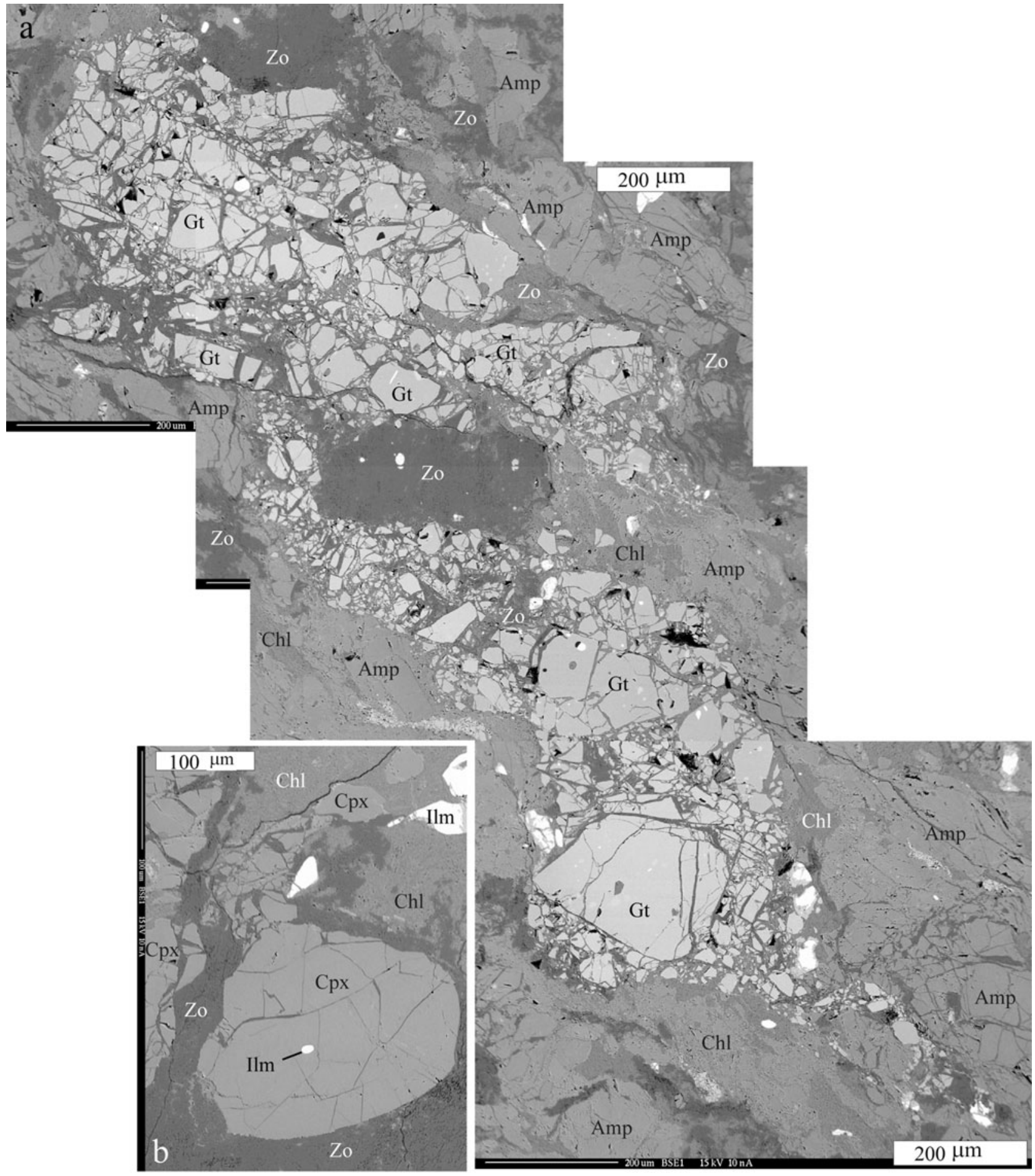

Figure 6. (a) Garnet grains and zoisite (pseudomorph of plagioclase) in garnet amphibolite, BSE; and (b) clinopyroxene with an ilmenite inclusion in garnet amphibolite, BSE. Mineral abbreviations as for Figure 5.

although most analyses are restricted to a range of $\mathrm{Py}_{10-25} \mathrm{Gro}_{25-30} \mathrm{Alm}_{55-75}$. Garnet in sample K26 contains relatively higher pyrope (mostly $>20 \mathrm{~mol} . \%$ ) and lower grossular component. All these garnet analyses fall within in the field corresponding to garnet in eclogite coexisting with blueschist (Fig. 8a). No compositional zoning has been found in garnet grains, although there is variation in some element contents from core to rim in different garnet grains (Fig. 8b-j).
Compositional variation of clinopyroxene in garnet amphibolite is listed in Supplementary Table 4 and shown in Supplementary Figure 3 (both available at http://journals.cambridge.org/geo). Clinopyroxene from sample $\mathrm{K} 24$ is rich in $\mathrm{Ca}$ (Wo>46 mol.\%) and located in the region of diopside-hedenbergite, while clinopyroxene from sample $\mathrm{K} 26$ is rich in $\mathrm{Mg}$ $(\mathrm{En}>33 \mathrm{~mol} . \%) . \mathrm{Na}_{2} \mathrm{O}$ contents in clinopyroxene from sample K26 (>0.4 wt \%) are obviously higher than that in sample K24 $(<0.4 \mathrm{wt} \%)$. Amphibole 

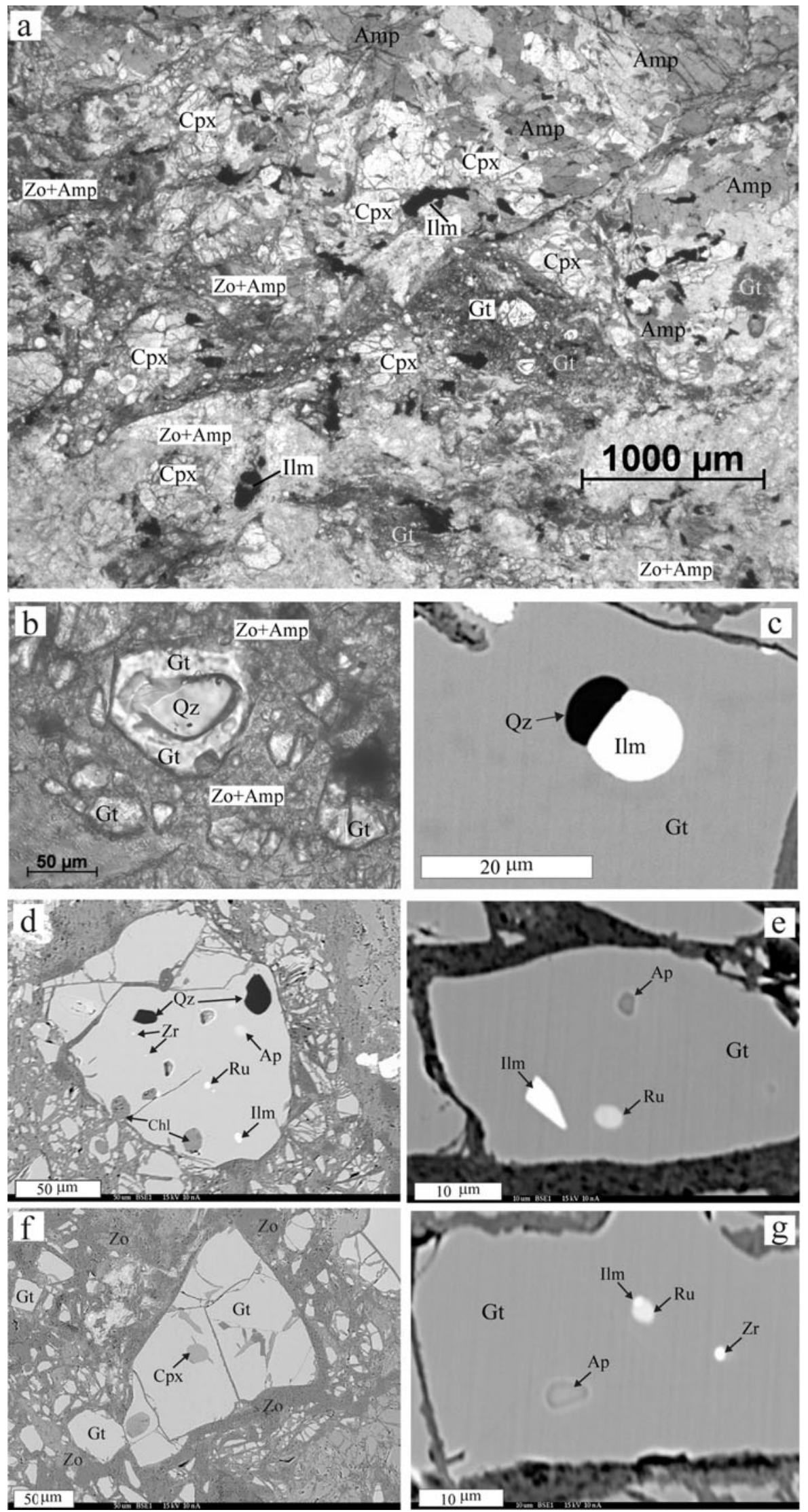

Figure 7. (a) Fragmentized garnet grain; plane-polarized light; (b) quartz inclusion in garnet; plane-polarized light; and (c-g) mineral inclusions in garnet, BSE. Ap - apatite; Qz - quartz; Ru - rutile; $\mathrm{Zr}$ - zircon; other mineral abbreviations as for Figure 5. 
Table 3. Trace element concentrations of clinopyroxene in garnet amphibolite by LA-ICP-MS (in ppm).

\begin{tabular}{|c|c|c|c|c|c|c|c|c|c|c|c|c|c|c|}
\hline Spot & 1 & 2 & 3 & 4 & 5 & 6 & 7 & 8 & 9 & 10 & 11 & 12 & 13 & 14 \\
\hline $\mathrm{Sc}$ & 105 & 91.6 & 83.4 & 83.5 & 75.7 & 89.9 & 88.7 & 104 & 98.8 & 98.0 & 115 & 95.9 & 85.8 & 107 \\
\hline V & 465 & 475 & 367 & 383 & 304 & 436 & 407 & 503 & 497 & 522 & 555 & 499 & 417 & 503 \\
\hline $\mathrm{Cr}$ & 159 & 154 & 172 & 119 & 76.6 & 153 & 189 & 126 & 121 & 122 & 132 & 116 & 163 & 124 \\
\hline Co & 61.2 & 49.2 & 52.0 & 48.9 & 48.5 & 48.3 & 48.0 & 51.3 & 50.7 & 50.0 & 49.5 & 49.5 & 53.3 & 53.1 \\
\hline $\mathrm{Ni}$ & 79.2 & 58.8 & 60.3 & 57.2 & 57.7 & 57.4 & 56.0 & 70.7 & 72.6 & 71.4 & 70.7 & 73.3 & 76.0 & 73.9 \\
\hline $\mathrm{Ga}$ & 13.49 & 12.08 & 10.37 & 10.40 & 9.17 & 11.31 & 10.39 & 10.59 & 10.39 & 12.02 & 11.22 & 10.73 & 10.77 & 11.04 \\
\hline Cs & 1.06 & 0.28 & 0.29 & 0.13 & 0.007 & 0.007 & 0.042 & 0.15 & 0.077 & 0.25 & 0.13 & 0.093 & 0.749 & 0.33 \\
\hline $\mathrm{Rb}$ & 1.10 & 0.23 & 0.19 & 0.16 & 0.013 & 0.013 & 0.067 & 0.27 & 0.15 & 0.38 & 0.25 & 0.14 & 0.83 & 0.40 \\
\hline $\mathrm{Ba}$ & 2.43 & 0.64 & 1.16 & 3.87 & 0.53 & 0.720 & 1.33 & 1.33 & 1.31 & 2.16 & 2.19 & 2.63 & 5.45 & 3.06 \\
\hline Th & 0.024 & 0.021 & 0.018 & 0.025 & 0.022 & 0.015 & 0.030 & 0.016 & 0.036 & 0.037 & 0.024 & 0.022 & 0.018 & 0.014 \\
\hline $\mathrm{U}$ & 0.041 & 0.037 & 0.017 & 0.036 & 0.042 & 0.041 & 0.046 & 0.032 & 0.050 & 0.039 & 0.034 & 0.032 & 0.029 & 0.026 \\
\hline Тa & 0.013 & 0.016 & 0.008 & 0.011 & 0.003 & 0.011 & 0.006 & 0.004 & 0.007 & 0.007 & 0.009 & 0.008 & 0.005 & 0.006 \\
\hline $\mathrm{Nb}$ & 0.060 & 0.032 & 0.021 & 0.060 & 0.026 & 0.029 & 0.023 & 0.024 & 0.019 & 0.047 & 0.054 & 0.040 & 0.028 & 0.022 \\
\hline $\mathrm{La}$ & 2.82 & 1.70 & 1.26 & 1.62 & 1.27 & 1.68 & 1.47 & 1.66 & 1.71 & 1.99 & 1.68 & 1.77 & 1.68 & 1.79 \\
\hline $\mathrm{Ce}$ & 12.82 & 6.46 & 4.70 & 5.92 & 4.82 & 6.20 & 5.51 & 6.88 & 7.06 & 8.39 & 7.15 & 7.27 & 6.78 & 7.44 \\
\hline $\mathrm{Pb}$ & 0.054 & 0.089 & 0.087 & 0.10 & 0.12 & 0.096 & 0.10 & 0.12 & 0.075 & 0.14 & 0.061 & 0.062 & 0.062 & 0.077 \\
\hline $\mathrm{Sr}$ & 8.97 & 5.15 & 4.71 & 6.52 & 4.24 & 4.43 & 4.82 & 5.20 & 4.97 & 7.43 & 6.57 & 6.89 & 8.20 & 6.01 \\
\hline $\mathrm{Nd}$ & 17.23 & 6.79 & 4.83 & 5.73 & 5.06 & 6.35 & 5.66 & 8.43 & 8.71 & 10.20 & 9.05 & 8.99 & 8.11 & 9.10 \\
\hline $\mathrm{Zr}$ & 52.44 & 61.41 & 38.84 & 47.26 & 36.60 & 54.17 & 45.83 & 47.53 & 47.84 & 64.84 & 55.44 & 51.49 & 42.79 & 50.06 \\
\hline $\mathrm{Hf}$ & 2.73 & 2.30 & 1.67 & 2.02 & 1.57 & 2.24 & 2.12 & 2.46 & 2.45 & 3.04 & 2.72 & 2.64 & 2.06 & 2.68 \\
\hline $\mathrm{Ti}$ & 1954 & 2122 & 1328 & 1863 & 1360 & 1975 & 1588 & 1605 & 1625 & 2215 & 1961 & 1819 & 1485 & 1781 \\
\hline $\mathrm{Sm}$ & 6.70 & 2.37 & 1.79 & 2.10 & 1.77 & 2.34 & 2.06 & 3.34 & 3.34 & 4.12 & 3.60 & 3.55 & 3.14 & 3.47 \\
\hline $\mathrm{Eu}$ & 1.82 & 0.84 & 0.66 & 0.74 & 0.62 & 0.84 & 0.76 & 0.94 & 0.99 & 1.19 & 1.09 & 1.05 & 0.95 & 1.04 \\
\hline Y & 43.42 & 18.78 & 13.16 & 17.32 & 13.24 & 17.38 & 14.61 & 29.20 & 30.34 & 35.44 & 34.33 & 31.22 & 27.21 & 31.24 \\
\hline $\mathrm{Lu}$ & 0.48 & 0.35 & 0.27 & 0.38 & 0.27 & 0.34 & 0.29 & 0.58 & 0.56 & 0.66 & 0.64 & 0.61 & 0.59 & 0.61 \\
\hline $\operatorname{Pr}$ & 2.64 & 1.15 & 0.83 & 1.02 & 0.88 & 1.09 & 0.98 & 1.33 & 1.38 & 1.63 & 1.43 & 1.42 & 1.32 & 1.43 \\
\hline $\mathrm{Gd}$ & 9.15 & 2.87 & 2.06 & 2.61 & 2.07 & 2.78 & 2.48 & 4.39 & 4.57 & 5.42 & 4.94 & 4.53 & 3.94 & 4.71 \\
\hline $\mathrm{Tb}$ & 1.62 & 0.57 & 0.43 & 0.51 & 0.39 & 0.54 & 0.46 & 0.89 & 0.95 & 1.07 & 1.03 & 0.94 & 0.85 & 0.97 \\
\hline Dy & 9.32 & 3.66 & 2.70 & 3.21 & 2.62 & 3.39 & 2.98 & 5.98 & 6.24 & 7.09 & 6.95 & 6.35 & 5.46 & 6.33 \\
\hline Но & 1.80 & 0.81 & 0.55 & 0.74 & 0.58 & 0.74 & 0.65 & 1.33 & 1.37 & 1.60 & 1.50 & 1.39 & 1.23 & 1.39 \\
\hline $\mathrm{Er}$ & 4.37 & 2.13 & 1.56 & 2.05 & 1.59 & 2.00 & 1.71 & 3.57 & 3.69 & 4.28 & 4.21 & 3.73 & 3.27 & 3.83 \\
\hline $\mathrm{Tm}$ & 0.56 & 0.32 & 0.25 & 0.33 & 0.23 & 0.31 & 0.29 & 0.52 & 0.55 & 0.65 & 0.65 & 0.57 & 0.51 & 0.57 \\
\hline $\mathrm{Yb}$ & 3.50 & 2.33 & 1.57 & 2.47 & 1.62 & 2.20 & 1.91 & 3.75 & 3.92 & 4.54 & 4.23 & 3.93 & 3.71 & 4.10 \\
\hline$(\mathrm{Ce} / \mathrm{Yb})_{\mathrm{N}}$ & 1.02 & 0.77 & 0.83 & 0.67 & 0.83 & 0.78 & 0.80 & 0.51 & 0.50 & 0.51 & 0.47 & 0.51 & 0.51 & 0.50 \\
\hline$(\mathrm{Gd} / \mathrm{Yb})_{\mathrm{N}}$ & 2.16 & 1.02 & 1.09 & 0.87 & 1.06 & 1.05 & 1.07 & 0.97 & 0.96 & 0.99 & 0.97 & 0.95 & 0.88 & 0.95 \\
\hline $\mathrm{Eu} / \mathrm{Eu} *$ & 0.71 & 0.99 & 1.04 & 0.96 & 0.98 & 1.00 & 1.02 & 0.75 & 0.77 & 0.77 & 0.79 & 0.80 & 0.82 & 0.78 \\
\hline
\end{tabular}


Table 3. (Continued)

\begin{tabular}{|c|c|c|c|c|c|c|c|c|c|c|c|c|c|c|c|}
\hline Spot & 15 & 16 & 17 & 18 & 19 & 20 & 21 & 22 & 23 & 24 & 25 & 26 & 27 & 28 & 29 \\
\hline $\mathrm{Sc}$ & 71.6 & 70.4 & 73.2 & 75.8 & 65.5 & 73.5 & 72.2 & 78.6 & 68.8 & 98.0 & 90.7 & 101 & 95.9 & 107 & 95.2 \\
\hline V & 356 & 396 & 359 & 369 & 330 & 434 & 430 & 433 & 357 & 489 & 451 & 458 & 478 & 420 & 441 \\
\hline $\mathrm{Cr}$ & 71.8 & 70.7 & 87.9 & 77.2 & 95.1 & 87.3 & 97.9 & 100 & 71.6 & 239 & 219 & 238 & 203 & 191 & 200 \\
\hline Co & 52.6 & 51.0 & 54.2 & 53.4 & 54.9 & 53.4 & 54.0 & 54.2 & 56.0 & 47.8 & 46.2 & 46.2 & 47.9 & 46.5 & 46.6 \\
\hline $\mathrm{Ni}$ & 60.0 & 56.5 & 63.2 & 61.9 & 58.0 & 61.4 & 62.0 & 63.3 & 61.3 & 61.6 & 58.4 & 58.4 & 62.1 & 58.2 & 58.6 \\
\hline $\mathrm{Ga}$ & 10.78 & 12.06 & 10.92 & 11.97 & 9.16 & 12.77 & 12.47 & 12.46 & 10.39 & 11.01 & 9.73 & 9.78 & 10.62 & 8.98 & 9.76 \\
\hline Cs & 0.26 & 0.25 & 0.008 & 0.087 & 0.94 & 0.008 & 0.14 & 0.027 & 0.27 & 0.063 & 0.12 & 0.068 & 0.019 & 0.058 & 0.14 \\
\hline $\mathrm{Rb}$ & 0.41 & 0.42 & 0.025 & 0.11 & 1.03 & 0.018 & 0.19 & 0.048 & 0.27 & 0.065 & 0.22 & 0.090 & 0.054 & 0.11 & 0.17 \\
\hline $\mathrm{Ba}$ & 3.44 & 5.47 & 0.78 & 1.96 & 6.43 & 0.52 & 1.84 & 0.74 & 1.40 & 0.69 & 4.12 & 1.63 & 0.49 & 2.08 & 4.37 \\
\hline Th & 0.014 & 0.026 & 0.028 & 0.018 & 0.140 & 0.039 & 0.032 & 0.037 & 0.035 & 0.027 & 0.020 & 0.021 & 0.016 & 0.018 & 0.020 \\
\hline $\mathrm{U}$ & 0.025 & 0.033 & 0.037 & 0.037 & 0.059 & 0.048 & 0.043 & 0.042 & 0.050 & 0.069 & 0.050 & 0.043 & 0.056 & 0.041 & 0.049 \\
\hline $\mathrm{Ta}$ & 0.011 & 0.014 & 0.021 & 0.008 & 0.005 & 0.011 & 0.012 & 0.008 & 0.011 & 0.007 & 0.006 & 0.007 & 0.010 & 0.006 & 0.006 \\
\hline $\mathrm{Nb}$ & 0.043 & 0.061 & 0.166 & 0.051 & 0.029 & 0.031 & 0.044 & 0.036 & 0.047 & 0.029 & 0.027 & 0.025 & 0.019 & 0.024 & 0.026 \\
\hline $\mathrm{La}$ & 2.04 & 2.11 & 2.14 & 2.23 & 1.60 & 2.40 & 2.34 & 2.33 & 2.04 & 1.61 & 1.41 & 1.30 & 1.43 & 1.16 & 1.30 \\
\hline $\mathrm{Ce}$ & 8.50 & 8.61 & 8.95 & 9.27 & 6.52 & 9.99 & 9.77 & 9.76 & 8.47 & 5.98 & 5.21 & 4.90 & 5.51 & 4.32 & 5.02 \\
\hline $\mathrm{Pb}$ & 0.044 & 0.038 & 0.058 & 0.072 & 0.065 & 0.086 & 0.051 & 0.13 & 0.097 & 0.21 & 0.18 & 0.20 & 0.18 & 0.15 & 0.16 \\
\hline $\mathrm{Sr}$ & 8.51 & 10.58 & 5.98 & 6.98 & 8.82 & 5.59 & 6.51 & 6.08 & 6.76 & 3.75 & 5.96 & 4.76 & 3.87 & 4.60 & 5.80 \\
\hline $\mathrm{Nd}$ & 8.91 & 9.10 & 9.39 & 9.58 & 6.60 & 10.47 & 10.17 & 10.23 & 8.71 & 6.86 & 6.03 & 5.32 & 6.36 & 5.01 & 5.68 \\
\hline $\mathrm{Zr}$ & 38.73 & 45.49 & 41.85 & 47.01 & 32.38 & 55.31 & 51.42 & 50.07 & 39.61 & 53.38 & 45.51 & 44.73 & 53.77 & 39.88 & 44.32 \\
\hline $\mathrm{Hf}$ & 2.16 & 2.46 & 2.46 & 2.40 & 1.76 & 2.91 & 2.79 & 2.79 & 2.20 & 2.71 & 2.26 & 2.32 & 2.53 & 2.11 & 2.28 \\
\hline $\mathrm{Ti}$ & 1472 & 1712 & 1641 & 1796 & 1005 & 1894 & 1757 & 1816 & 1198 & 2021 & 1711 & 1636 & 1978 & 1550 & 1693 \\
\hline Sm & 3.03 & 3.16 & 3.07 & 3.20 & 2.18 & 3.46 & 3.28 & 3.37 & 2.98 & 2.45 & 2.17 & 2.06 & 2.43 & 2.05 & 2.15 \\
\hline $\mathrm{Eu}$ & 1.01 & 1.06 & 1.05 & 1.05 & 0.746 & 1.10 & 1.09 & 1.14 & 0.950 & 0.818 & 0.759 & 0.687 & 0.797 & 0.616 & 0.724 \\
\hline Y & 15.48 & 16.77 & 16.16 & 17.19 & 11.97 & 18.03 & 18.09 & 18.01 & 14.92 & 19.38 & 17.98 & 16.26 & 18.74 & 15.11 & 17.03 \\
\hline $\mathrm{Lu}$ & 0.24 & 0.27 & 0.23 & 0.26 & 0.21 & 0.25 & 0.25 & 0.25 & 0.21 & 0.41 & 0.46 & 0.34 & 0.38 & 0.329 & 0.421 \\
\hline $\operatorname{Pr}$ & 1.56 & 1.63 & 1.66 & 1.66 & 1.18 & 1.85 & 1.77 & 1.79 & 1.53 & 1.08 & 0.984 & 0.905 & 1.05 & 0.835 & 0.923 \\
\hline $\mathrm{Gd}$ & 3.16 & 3.50 & 3.45 & 3.40 & 2.34 & 3.73 & 3.83 & 3.58 & 3.12 & 3.07 & 2.76 & 2.49 & 3.13 & 2.23 & 2.69 \\
\hline $\mathrm{Tb}$ & 0.59 & 0.62 & 0.63 & 0.65 & 0.45 & 0.72 & 0.69 & 0.69 & 0.61 & 0.63 & 0.59 & 0.51 & 0.62 & 0.47 & 0.558 \\
\hline Dy & 3.62 & 3.91 & 3.86 & 4.03 & 2.81 & 4.33 & 4.33 & 4.33 & 3.63 & 4.17 & 3.77 & 3.40 & 4.02 & 3.19 & 3.61 \\
\hline Но & 0.73 & 0.76 & 0.76 & 0.81 & 0.56 & 0.86 & 0.87 & 0.84 & 0.72 & 0.94 & 0.82 & 0.74 & 0.85 & 0.68 & 0.79 \\
\hline $\mathrm{Er}$ & 1.69 & 1.90 & 1.86 & 1.95 & 1.34 & 2.04 & 1.99 & 2.00 & 1.71 & 2.46 & 2.36 & 2.08 & 2.37 & 1.95 & 2.17 \\
\hline $\mathrm{Tm}$ & 0.24 & 0.27 & 0.24 & 0.27 & 0.20 & 0.28 & 0.28 & 0.28 & 0.24 & 0.36 & 0.38 & 0.31 & 0.35 & 0.28 & 0.35 \\
\hline $\mathrm{Yb}$ & 1.65 & 1.85 & 1.64 & 1.70 & 1.33 & 1.91 & 1.78 & 1.76 & 1.47 & 2.59 & 2.80 & 2.21 & 2.48 & 2.26 & 2.64 \\
\hline$(\mathrm{Ce} / \mathrm{Yb})_{\mathrm{N}}$ & 1.43 & 1.29 & 1.52 & 1.51 & 1.36 & 1.45 & 1.52 & 1.54 & 1.60 & 0.64 & 0.52 & 0.62 & 0.62 & 0.53 & 0.53 \\
\hline$(\mathrm{Gd} / \mathrm{Yb})_{\mathrm{N}}$ & 1.58 & 1.56 & 1.74 & 1.65 & 1.46 & 1.62 & 1.78 & 1.68 & 1.76 & 0.98 & 0.82 & 0.93 & 1.04 & 0.82 & 0.84 \\
\hline $\mathrm{Eu} / \mathrm{Eu} *$ & 0.99 & 0.97 & 0.98 & 0.97 & 1.01 & 0.93 & 0.94 & 1.00 & 0.95 & 0.91 & 0.95 & 0.92 & 0.88 & 0.88 & 0.92 \\
\hline
\end{tabular}


Table 4. Trace element concentrations of garnet in amphibolite by LA-ICP-MS (in ppm).

\begin{tabular}{|c|c|c|c|c|c|c|c|c|c|c|c|c|c|}
\hline Spot & 1 & 2 & 3 & 4 & 5 & 6 & 7 & 8 & 9 & 10 & 11 & 12 & 13 \\
\hline $\mathrm{Sc}$ & 79.4 & 72.3 & 90.9 & 144 & 152 & 95.4 & 111 & 107 & 115 & 128 & 107 & 82.9 & 118 \\
\hline $\mathrm{V}$ & 227 & 211 & 184 & 104 & 116 & 86.1 & 86 & 169 & 127 & 134 & 124 & 106 & 294 \\
\hline $\mathrm{Cr}$ & 72.4 & 63.5 & 128 & 101 & 129 & 61.6 & 59.6 & 97.5 & 61.7 & 83.9 & 100 & 298 & 72.5 \\
\hline Co & 50.0 & 50.4 & 52.1 & 23.5 & 32.6 & 20.9 & 22.2 & 40.5 & 41.0 & 40.9 & 43.2 & 42.1 & 33.4 \\
\hline $\mathrm{Ni}$ & 2.1 & 2.5 & 2.2 & 0.17 & 17.0 & 3.7 & 6.1 & 2.2 & 2.0 & 1.8 & 2.0 & 1.6 & 2.0 \\
\hline $\mathrm{Ga}$ & 12.7 & 12.9 & 10.9 & 6.0 & 7.4 & 5.5 & 5.7 & 5.7 & 5.2 & 5.0 & 6.2 & 6.6 & 7.3 \\
\hline Cs & 0.014 & 0.019 & 0.014 & 0.016 & 0.12 & 0.026 & 0.042 & 0.014 & 0.016 & 0.014 & 0.011 & 0.015 & 0.012 \\
\hline $\mathrm{Rb}$ & 0.041 & 0.038 & 0.069 & 0.15 & 0.16 & 0.084 & 0.11 & 0.044 & 0.027 & 0.034 & 0.030 & 0.035 & 0.024 \\
\hline $\mathrm{Ba}$ & 0.067 & 0.072 & 0.10 & 0.088 & 0.19 & 0.11 & 0.076 & 0.086 & 0.079 & 0.056 & 0.078 & 0.083 & 0.055 \\
\hline Th & 0.008 & 0.012 & 0.008 & 0.011 & 0.013 & 0.008 & 0.012 & 0.008 & 0.019 & 0.007 & 0.010 & 0.014 & 0.011 \\
\hline U & 0.014 & 0.016 & 0.012 & 0.015 & 0.014 & 0.012 & 0.113 & 0.034 & 0.026 & 0.025 & 0.010 & 0.015 & 0.066 \\
\hline $\mathrm{Ta}$ & 0.007 & 0.006 & 0.006 & 0.009 & 0.015 & 0.008 & 0.011 & 0.008 & 0.011 & 0.006 & 0.005 & 0.006 & 0.004 \\
\hline $\mathrm{Nb}$ & 0.006 & 0.015 & 0.011 & 0.010 & 0.013 & 0.075 & 0.12 & 0.011 & 0.19 & 0.025 & 0.006 & 0.013 & 0.047 \\
\hline $\mathrm{La}$ & 0.009 & 0.013 & 0.008 & 0.013 & 0.012 & 0.007 & 0.008 & 0.010 & 0.007 & 0.007 & 0.011 & 0.010 & 0.010 \\
\hline $\mathrm{Ce}$ & 0.093 & 0.108 & 0.084 & 0.027 & 0.041 & 0.041 & 0.056 & 0.103 & 0.045 & 0.075 & 0.044 & 0.038 & 0.125 \\
\hline $\mathrm{Pb}$ & 0.036 & 0.04 & 0.055 & 0.045 & 0.062 & 0.031 & 0.038 & 0.45 & 0.029 & 0.028 & 0.13 & 0.031 & 0.024 \\
\hline $\mathrm{Sr}$ & 0.051 & 0.027 & 0.041 & 0.075 & 0.42 & 0.65 & 0.19 & 0.54 & 0.014 & 0.013 & 0.030 & 0.014 & 0.032 \\
\hline $\mathrm{Nd}$ & 1.26 & 1.49 & 1.21 & 0.37 & 0.39 & 0.42 & 0.47 & 0.94 & 0.55 & 0.71 & 0.46 & 0.32 & 1.52 \\
\hline $\mathrm{Zr}$ & 12.87 & 13.59 & 12.82 & 12.1 & 10.88 & 14.08 & 98.82 & 16.72 & 22.95 & 14.98 & 15.70 & 13.82 & 22.56 \\
\hline $\mathrm{Hf}$ & 0.21 & 0.20 & 0.18 & 0.15 & 0.12 & 0.12 & 1.88 & 0.18 & 0.24 & 0.16 & 0.14 & 0.17 & 0.32 \\
\hline $\mathrm{Ti}$ & 373 & 424 & 272 & 274 & 262 & 328 & 410 & 511 & 740 & 529 & 287 & 277 & 756 \\
\hline $\mathrm{Sm}$ & 3.45 & 4.01 & 3.15 & 0.75 & 0.73 & 1.05 & 1.21 & 1.26 & 0.97 & 1.14 & 0.81 & 0.78 & 2.02 \\
\hline $\mathrm{Eu}$ & 1.73 & 2.05 & 1.67 & 0.46 & 0.50 & 0.82 & 0.88 & 0.83 & 0.71 & 0.71 & 0.65 & 0.57 & 1.10 \\
\hline Y & 289 & 234 & 387 & 654 & 278 & 213 & 245 & 143 & 173 & 182 & 132 & 105 & 66.2 \\
\hline $\mathrm{Lu}$ & 5.93 & 4.29 & 8.95 & 21.02 & 13.11 & 4.81 & 5.41 & 2.49 & 3.39 & 4.55 & 2.90 & 3.14 & 1.40 \\
\hline $\operatorname{Pr}$ & 0.068 & 0.065 & 0.059 & 0.018 & 0.021 & 0.031 & 0.035 & 0.061 & 0.025 & 0.055 & 0.027 & 0.025 & 0.075 \\
\hline $\mathrm{Gd}$ & 16.93 & 18.81 & 17.06 & 3.24 & 3.61 & 8.83 & 9.92 & 3.74 & 3.87 & 2.94 & 3.92 & 3.37 & 2.74 \\
\hline $\mathrm{Tb}$ & 5.30 & 5.35 & 5.87 & 2.11 & 1.57 & 3.29 & 3.97 & 1.75 & 1.57 & 1.36 & 1.51 & 1.39 & 0.82 \\
\hline Dy & 42.05 & 37.14 & 52.75 & 43.94 & 22.08 & 31.17 & 36.94 & 18.68 & 19.01 & 18.43 & 15.88 & 14.16 & 7.99 \\
\hline Ho & 10.69 & 8.71 & 15.05 & 24.09 & 9.62 & 8.79 & 10.20 & 5.25 & 6.12 & 6.67 & 4.95 & 4.07 & 2.21 \\
\hline $\mathrm{Er}$ & 31.86 & 24.94 & 46.56 & 107.5 & 41.61 & 27.69 & 31.89 & 15.00 & 19.74 & 23.99 & 16.12 & 13.39 & 7.23 \\
\hline Tm & 5.16 & 3.92 & 7.87 & 20.45 & 8.72 & 4.62 & 5.17 & 2.28 & 3.14 & 3.94 & 2.63 & 2.33 & 1.26 \\
\hline $\mathrm{Yb}$ & 38.01 & 27.72 & 56.26 & 155.34 & 78.35 & 32.63 & 36.66 & 15.94 & 22.04 & 28.52 & 19.08 & 19.18 & 9.32 \\
\hline
\end{tabular}


Table 4. (Continued)

\begin{tabular}{|c|c|c|c|c|c|c|c|c|c|c|c|c|c|}
\hline Spot & 14 & 15 & 16 & 17 & 18 & 19 & 20 & 21 & 22 & 23 & 24 & 25 & 26 \\
\hline $\mathrm{Sc}$ & 104 & 98.3 & 91.9 & 101 & 103 & 88.8 & 116 & 85.4 & 103 & 107 & 109 & 98.6 & 95.6 \\
\hline V & 112 & 118 & 125 & 117 & 141 & 94.1 & 118 & 117 & 145 & 105 & 203 & 105 & 99.9 \\
\hline $\mathrm{Cr}$ & 93.8 & 58.4 & 162 & 72.9 & 90.6 & 161 & 78.7 & 95.4 & 83.7 & 71.2 & 117 & 71.8 & 78.2 \\
\hline Co & 41.9 & 42.3 & 44.5 & 42.4 & 42.4 & 42.2 & 37.3 & 37.0 & 38.9 & 37.3 & 32.2 & 28.3 & 35.8 \\
\hline $\mathrm{Ni}$ & 1.4 & 2.6 & 1.5 & 3.7 & 1.9 & 1.7 & 1.8 & 1.7 & 10.0 & 1.6 & 3.1 & 2.5 & 2.1 \\
\hline $\mathrm{Ga}$ & 6.02 & 5.61 & 6.70 & 5.70 & 6.01 & 5.14 & 5.01 & 4.95 & 5.81 & 5.06 & 6.48 & 5.05 & 4.94 \\
\hline Cs & 0.012 & 0.015 & 0.017 & 0.104 & 0.009 & 0.010 & 0.013 & 0.014 & 0.129 & 0.012 & 0.065 & 0.074 & 0.012 \\
\hline $\mathrm{Rb}$ & 0.029 & 0.048 & 0.057 & 0.095 & 0.025 & 0.027 & 0.046 & 0.036 & 0.17 & 0.028 & 0.15 & 0.17 & 0.033 \\
\hline $\mathrm{Ba}$ & 0.055 & 0.046 & 0.082 & 0.080 & 0.055 & 0.064 & 0.053 & 0.057 & 0.56 & 0.042 & 0.22 & 0.19 & 0.028 \\
\hline Th & 0.010 & 0.006 & 0.009 & 0.010 & 0.007 & 0.008 & 0.004 & 0.012 & 0.007 & 0.049 & 0.009 & 0.27 & 0.008 \\
\hline U & 0.010 & 0.018 & 0.009 & 0.023 & 0.028 & 0.012 & 0.019 & 0.014 & 0.025 & 0.48 & 0.053 & 0.91 & 0.022 \\
\hline $\mathrm{Ta}$ & 0.005 & 0.009 & 0.007 & 0.007 & 0.017 & 0.008 & 0.007 & 0.004 & 0.007 & 0.008 & 0.081 & 0.042 & 0.003 \\
\hline $\mathrm{Nb}$ & 0.016 & 0.006 & 0.009 & 0.019 & 0.22 & 0.005 & 0.013 & 0.009 & 0.009 & 0.013 & 0.86 & 0.76 & 0.008 \\
\hline $\mathrm{La}$ & 0.008 & 0.006 & 0.013 & 0.011 & 0.025 & 0.007 & 0.009 & 0.007 & 0.011 & 0.008 & 0.007 & 1.47 & 0.009 \\
\hline $\mathrm{Ce}$ & 0.028 & 0.041 & 0.040 & 0.054 & 0.094 & 0.046 & 0.056 & 0.047 & 0.062 & 0.050 & 0.075 & 3.80 & 0.10 \\
\hline $\mathrm{Pb}$ & 0.032 & 0.043 & 0.038 & 0.050 & 0.021 & 0.065 & 0.024 & 0.037 & 0.13 & 0.027 & 0.028 & 0.030 & 0.023 \\
\hline $\mathrm{Sr}$ & 0.023 & 0.039 & 0.051 & 0.180 & 0.016 & 0.017 & 0.025 & 0.020 & 0.50 & 0.018 & 1.84 & 1.44 & 0.011 \\
\hline $\mathrm{Nd}$ & 0.37 & 0.59 & 0.43 & 0.60 & 0.43 & 0.45 & 0.67 & 0.61 & 0.58 & 0.51 & 0.67 & 1.92 & 0.77 \\
\hline $\mathrm{Zr}$ & 14.38 & 17.05 & 10.83 & 16.56 & 13.83 & 16.65 & 17.47 & 17.51 & 13.85 & 1018 & 21.08 & 17.82 & 10.30 \\
\hline $\mathrm{Hf}$ & 0.13 & 0.13 & 0.15 & 0.13 & 0.14 & 0.14 & 0.16 & 0.17 & 0.16 & 24.65 & 0.36 & 0.34 & 0.08 \\
\hline $\mathrm{Ti}$ & 257 & 341 & 289 & 408 & 756 & 292 & 401 & 350 & 384 & 304 & 71.1 & 2083 & 458 \\
\hline $\mathrm{Sm}$ & 0.74 & 1.16 & 0.78 & 1.21 & 0.92 & 0.84 & 1.04 & 1.04 & 0.86 & 0.95 & 0.65 & 0.27 & 0.85 \\
\hline $\mathrm{Eu}$ & 0.59 & 0.71 & 0.63 & 0.82 & 0.62 & 0.67 & 0.72 & 0.74 & 0.54 & 0.65 & 0.45 & 0.14 & 0.50 \\
\hline $\mathrm{Y}$ & 126 & 159 & 136 & 137 & 101 & 105 & 208 & 112 & 176 & 181 & 64.1 & 74.9 & 95.1 \\
\hline $\mathrm{Lu}$ & 2.71 & 1.94 & 3.96 & 1.63 & 2.36 & 2.22 & 3.97 & 0.87 & 2.65 & 3.31 & 3.16 & 2.04 & 2.47 \\
\hline $\mathrm{Pr}$ & 0.022 & 0.026 & 0.033 & 0.040 & 0.037 & 0.027 & 0.031 & 0.033 & 0.036 & 0.021 & 0.040 & 0.488 & 0.048 \\
\hline $\mathrm{Gd}$ & 3.61 & 3.82 & 3.79 & 3.97 & 3.15 & 3.99 & 3.27 & 4.08 & 2.24 & 3.94 & 1.36 & 0.91 & 1.65 \\
\hline $\mathrm{Tb}$ & 1.43 & 1.56 & 1.49 & 1.76 & 1.10 & 1.66 & 1.58 & 1.77 & 1.31 & 1.77 & 0.48 & 0.51 & 0.63 \\
\hline Dy & 16.00 & 19.61 & 16.55 & 19.96 & 11.62 & 16.30 & 21.57 & 17.80 & 18.39 & 22.34 & 5.68 & 7.34 & 8.76 \\
\hline Ho & 5.00 & 6.08 & 5.28 & 5.45 & 3.81 & 4.30 & 7.61 & 4.24 & 6.19 & 7.08 & 2.07 & 3.07 & 3.35 \\
\hline $\mathrm{Er}$ & 15.83 & 17.03 & 18.11 & 14.50 & 13.00 & 12.04 & 24.93 & 9.91 & 19.40 & 21.72 & 9.03 & 11.08 & 12.35 \\
\hline Tm & 2.49 & 2.38 & 3.19 & 1.97 & 2.17 & 1.84 & 3.94 & 1.25 & 2.99 & 3.36 & 2.04 & 1.95 & 2.20 \\
\hline $\mathrm{Yb}$ & 18.23 & 15.11 & 25.18 & 12.51 & 15.65 & 13.47 & 27.38 & 7.09 & 19.92 & 23.26 & 18.67 & 13.89 & 16.46 \\
\hline
\end{tabular}



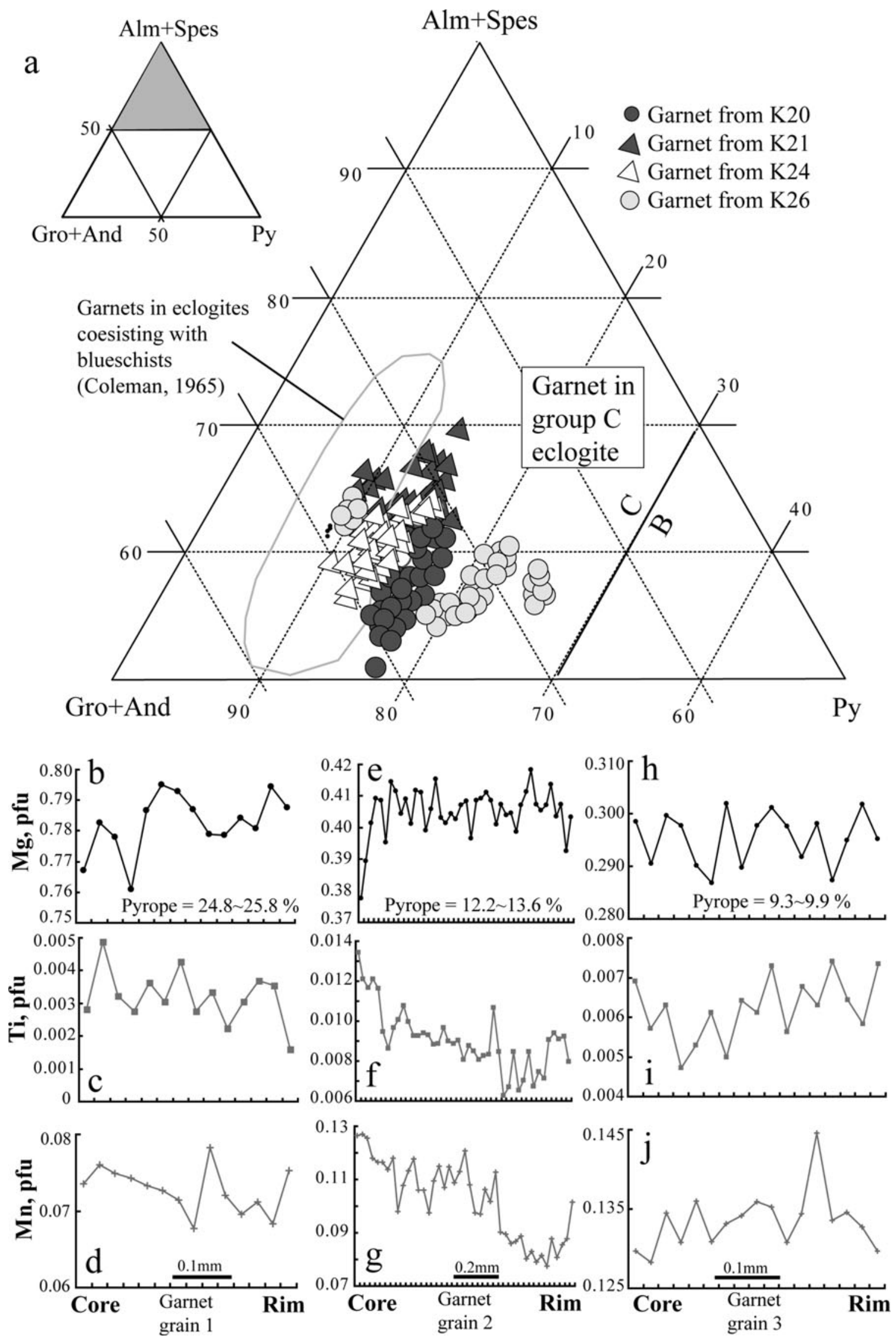

Figure 8. Plots showing variations of garnet in garnet amphibolite: (a) triangle plot showing compositions of garnet; Group C region is based on Coleman (1965); and (b-j) Mg, Ti and Mn variations across three garnet grains (corresponding to different pyrope components varying from $25.8 \mathrm{~mol} . \%$ to $9.3 \mathrm{~mol} . \%$ ). 
Table 5. The trace element contents of metagabbro and amphibolite in Baikouquan ophiolitic mélange (in ppm).

\begin{tabular}{|c|c|c|c|c|c|c|c|c|c|c|c|c|c|c|c|c|c|c|c|c|c|c|c|}
\hline $\begin{array}{l}\text { Sample } \\
\text { Rock* }\end{array}$ & $\begin{array}{l}\text { K33 } \\
\text { MG }\end{array}$ & $\begin{array}{c}\text { Bk36 } \\
\text { MG }\end{array}$ & $\begin{array}{l}\text { Bk6 } \\
\text { MG }\end{array}$ & $\begin{array}{l}\text { Bk7 } \\
\text { MG }\end{array}$ & $\begin{array}{c}\text { Bk13 } \\
\text { MG }\end{array}$ & $\begin{array}{l}\mathrm{J} 115 \\
\mathrm{MG}\end{array}$ & $\begin{array}{c}\mathrm{J}-116 \\
\mathrm{MG}\end{array}$ & $\begin{array}{c}\text { DJ101 } \\
\text { MG }\end{array}$ & $\begin{array}{c}\text { K20 } \\
\text { G-amp }\end{array}$ & $\begin{array}{c}\text { K21 } \\
\text { G-amp }\end{array}$ & $\begin{array}{c}\text { K24 } \\
\text { G-amp }\end{array}$ & $\begin{array}{c}\text { K26 } \\
\text { G-amp }\end{array}$ & $\begin{array}{l}\text { K19 } \\
\text { Amp }\end{array}$ & $\begin{array}{l}\text { K28 } \\
\text { Amp }\end{array}$ & $\begin{array}{l}\text { K22 } \\
\text { Amp }\end{array}$ & $\begin{array}{c}\text { K22-1 } \\
\text { Amp }\end{array}$ & $\begin{array}{l}\text { K27 } \\
\text { Amp }\end{array}$ & $\begin{array}{l}\text { XJ30 } \\
\text { Amp }\end{array}$ & $\begin{array}{l}\text { XJ43 } \\
\text { Amp }\end{array}$ & $\begin{array}{l}\text { XJ50 } \\
\text { Amp }\end{array}$ & $\begin{array}{l}\text { XJ65 } \\
\text { Amp }\end{array}$ & $\begin{array}{l}\text { XJ66 } \\
\text { Amp }\end{array}$ & $\begin{array}{l}\text { XJ68 } \\
\text { Amp }\end{array}$ \\
\hline $\mathrm{Li}$ & 40.85 & 10.60 & 46.40 & 34.20 & 57.80 & 52.92 & 37.47 & 53.49 & 5.67 & 6.11 & 4.81 & 14.1 & 8.35 & 7.10 & 5.67 & 6.11 & 8.86 & 6.91 & 15.1 & 13.1 & 8.13 & 14.8 & 5.88 \\
\hline $\mathrm{Be}$ & 0.098 & 0.056 & 0.067 & 0.39 & 1.19 & 0.09 & 0.20 & 0.09 & 0.24 & 0.27 & 0.32 & 0.43 & 0.41 & 0.34 & 0.24 & 0.27 & 0.52 & 0.42 & 0.37 & 0.52 & 0.47 & 0.48 & 0.46 \\
\hline $\mathrm{Sc}$ & 26.0 & 15.7 & 39.3 & 39.4 & 21.6 & 35.1 & 55.3 & 38.2 & 70.4 & 73.5 & 54.01 & 65.3 & 53.21 & 37.25 & 70.4 & 73.5 & 67.7 & 43.5 & 43.9 & 60.0 & 45.0 & 62.0 & 52.7 \\
\hline V & 94.21 & 58.7 & 71.5 & 86.2 & 30.0 & 101 & 147 & 104 & 442 & 433 & 388 & 462 & 421 & 297 & 442 & 433 & 427 & 356 & 311 & 394 & 325 & 374 & 335 \\
\hline $\mathrm{Cr}$ & 524 & 449 & 849 & 486 & 576 & 323 & 326 & 321 & 166 & 171 & 294 & 180 & 225 & 240 & 166 & 171 & 277 & 129 & 124 & 288 & 130 & 394 & 342 \\
\hline Co & 37.4 & 63.9 & 36.5 & 27.6 & 34.2 & 34.8 & 41.9 & 35.2 & 69.5 & 72.0 & 54.4 & 65.0 & 60.2 & 39.2 & 69.5 & 72.0 & 70.0 & 38.4 & 44.1 & 60.8 & 45.1 & 66.2 & 55.8 \\
\hline $\mathrm{Ni}$ & 135 & 300 & 134 & 159 & 143 & 129 & 131 & 124 & 94.3 & 97.6 & 108 & 84.2 & 94.72 & 62.08 & 94.3 & 97.6 & 121 & 37.7 & 45.7 & 154 & 47.7 & 202 & 151 \\
\hline $\mathrm{Cu}$ & 3.08 & 35.4 & 7.53 & 6.10 & 7.16 & 8.06 & 1.65 & 8.02 & 51.8 & 43.2 & 67.6 & 108 & 234 & 65.06 & 51.8 & 43.2 & 138 & 224 & 33.3 & 66.3 & 22.6 & 40.1 & 31.1 \\
\hline $\mathrm{Ga}$ & 13.77 & 9.33 & 12.2 & 9.14 & 7.16 & 9.10 & 7.96 & 9.10 & 11.7 & 11.6 & 11.3 & 10.9 & 17.43 & 17.43 & 11.7 & 11.6 & 13.2 & 13.5 & 10.5 & 12.2 & 11.2 & 10.5 & 17.1 \\
\hline $\mathrm{Rb}$ & 11.08 & 13.61 & 17.42 & 11.30 & 19.11 & 34.02 & 9.12 & 34.03 & 1.75 & 1.27 & 1.35 & 1.33 & 1.29 & 0.071 & 1.75 & 1.27 & 2.86 & 35.8 & 17.11 & 2.10 & 3.08 & 1.99 & 6.39 \\
\hline $\mathrm{Sr}$ & 256 & 229 & 894 & 750 & 900 & 204 & 275 & 201 & 265 & 265 & 294 & 56.11 & 83.90 & 224 & 265 & 265 & 112 & 440 & 125 & 74.62 & 150 & 63.10 & 81.81 \\
\hline Y & 4.16 & 2.37 & 2.85 & 3.78 & 1.82 & 2.28 & 3.02 & 2.78 & 33.70 & 34.10 & 27.20 & 36.80 & 36.90 & 24.88 & 33.70 & 34.10 & 30.50 & 28.50 & 21.40 & 33.20 & 19.70 & 38.90 & 28.30 \\
\hline $\mathrm{Zr}$ & 2.68 & 4.64 & 6.70 & 9.66 & 5.00 & 2.09 & 1.44 & 5.17 & 67.20 & 71.00 & 62.40 & 84.10 & 89.26 & 60.52 & 67.20 & 71.00 & 57.40 & 94.00 & 68.40 & 77.10 & 67.50 & 85.90 & 64.90 \\
\hline $\mathrm{Nb}$ & 0.37 & 0.075 & 0.24 & 0.16 & 0.062 & 0.15 & 0.15 & 0.17 & 3.91 & 4.01 & 3.77 & 5.02 & 7.02 & 1.06 & 3.91 & 4.01 & 7.98 & 1.66 & 1.35 & 5.77 & 1.78 & 5.14 & 3.89 \\
\hline $\mathrm{Cs}$ & 0.33 & 0.52 & 2.01 & 0.49 & 1.98 & 0.24 & 0.18 & 0.25 & 0.47 & 0.47 & 0.12 & 1.16 & 0.57 & 0.40 & 0.47 & 0.47 & 0.24 & 0.28 & 0.36 & 0.19 & 0.11 & 0.20 & 0.26 \\
\hline $\mathrm{Ba}$ & 65.81 & 86.00 & 352 & 193 & 444 & 1382 & 1010 & 1390 & 3.83 & 4.51 & 16.7 & 3.20 & 10.55 & 8.8 & 3.83 & 4.51 & 37.31 & 103 & 63.30 & 65.72 & 38.91 & 6.84 & 373 \\
\hline $\mathrm{La}$ & 0.13 & 0.31 & 0.31 & 0.23 & 0.21 & 0.16 & 0.28 & 0.13 & 3.87 & 4.05 & 4.42 & 4.09 & 7.52 & & 3.87 & 4.05 & 5.08 & 4.97 & 5.02 & 6.02 & 4.97 & 9.68 & 3.69 \\
\hline $\mathrm{Ce}$ & 0.34 & 0.64 & 0.78 & 0.70 & 0.54 & 0.47 & 0.64 & 0.34 & 9.79 & 10.41 & 10.51 & 11.70 & 18.01 & 8.8 & 9.79 & 10.40 & 11.71 & 12.02 & 12.10 & 14.22 & 11.80 & 22.51 & 9.22 \\
\hline Pr & 0.062 & 0.11 & 0.17 & 0.13 & 0.10 & 0.06 & 0.09 & 0.06 & 1.91 & 1.91 & 1.95 & 2.09 & 2.88 & 1.5 & 1.91 & 1.91 & 2.19 & 2.11 & 2.17 & 2.31 & 1.94 & 3.63 & 1.67 \\
\hline $\mathrm{Nd}$ & 0.37 & 0.58 & 0.95 & 0.97 & 0.4 & 0.41 & 0.58 & 0.40 & 9.65 & 9.93 & 9.19 & 11.51 & 14.54 & 8.1 & 9.65 & 9.93 & 10.70 & 11.52 & 10.21 & 12.10 & 9.53 & 17.81 & 8.34 \\
\hline $\mathrm{Sm}$ & 0.26 & 0.27 & & 0.37 & 0.1 & 0.2 & 0.26 & 0.20 & 2.80 & 2.99 & 2.49 & 3.48 & 4.20 & 2.6 & 2.8 & 2.99 & 3.15 & 3.19 & 2.74 & 3.46 & 2.62 & 4.62 & 2.86 \\
\hline $\mathrm{Eu}$ & 0.53 & 0.23 & 0.33 & 0.25 & 0.11 & 0.49 & 0.48 & 0.34 & 1.01 & 1.09 & 0.84 & 1.27 & 1.45 & 1.0 & 1.01 & 1.09 & 1.05 & 1.12 & 0.95 & 1.15 & 0.85 & 1.50 & 1.09 \\
\hline $\mathrm{Gd}$ & 0.54 & & & 0.50 & 0.28 & & 0.45 & & & & & 4.6 & 5.22 & & 4.1 & 3.9 & 4.36 & & & & & 5.53 & 3.93 \\
\hline $\mathrm{Tb}$ & 0.096 & 0.053 & 0.070 & 0.094 & 0.052 & 0.069 & 0.091 & 0.069 & 0.82 & 0.85 & 0.67 & 0.93 & 0.99 & 0.6 & 0.82 & 0.85 & 0.82 & 0.79 & 0.58 & 0.90 & 0.57 & 1.10 & 0.75 \\
\hline Dy & & & & 0.68 & 0.34 & 0.44 & 0.58 & & & & 4.57 & 6.5 & 6.45 & & & 5.8 & 5.48 & 5.1 & 3.80 & & 3.6 & 7.21 & 4.90 \\
\hline Ho & 0.14 & 0.088 & 0.12 & 0.14 & 0.071 & 0.092 & 0.12 & 0.089 & 1.30 & 1.33 & 1.01 & 1.47 & 1.44 & 0.96 & 1.30 & 1.33 & 1.21 & 1.13 & 0.85 & 1.37 & 0.79 & 1.55 & 1.17 \\
\hline $\mathrm{Er}$ & & & & & & & & & & & & & & & 3. & 3.8 & 3.3 & 3.2 & & & & 4.53 & 3.24 \\
\hline $\mathrm{Tm}$ & 0.068 & 0.047 & 0.051 & 0.071 & 0.031 & 0.042 & 0.051 & 0.041 & 0.66 & 0.61 & 0.47 & 0.6 & 0.60 & 0.4 & 0.6 & 0.6 & 0.54 & 0.52 & 0.39 & 0. & 0.35 & 0.71 & 0.51 \\
\hline $\mathrm{Yb}$ & 0.43 & 0.29 & 0.29 & 0.40 & 0.16 & 0.24 & 0.32 & 0. & 4. & & 3. & 4.6 & 3. & & 4.4 & 4. & 3.65 & 3.47 & 2.62 & 4. & 2.44 & 4.58 & 3.63 \\
\hline $\mathrm{Lu}$ & 0.056 & 0.045 & 0.045 & 0.062 & 0.022 & 0.041 & 0.051 & 0.035 & 0.6 & & 0.4 & 0.6 & 0.5 & & 0.6 & 0.6 & 0.52 & 0.49 & 0.37 & 0. & 0.37 & 0.66 & 0.51 \\
\hline $\mathrm{Hf}$ & 0.14 & 0.15 & 0.2 & 0.40 & 0.18 & 0.091 & 0.090 & & 2.1 & & 1.95 & 2.6 & 2.69 & & 2.1 & 2.2 & 2.02 & 2.7 & 2.2 & 2.47 & 2.13 & 2.60 & 2.04 \\
\hline $\mathrm{Ta}$ & & 0.027 & & 0.37 & & 0.061 & 0.081 & & 0.2 & & 0.29 & 0.3 & 0.68 & & 0.2 & 0.3 & 0.42 & 0.1 & 0.13 & & 0.1 & 0.33 & 0.27 \\
\hline Th & 0.11 & 0.062 & 0.081 & 0.022 & 0.032 & 0.021 & 0.022 & 0.02 & 0.19 & 0.1 & 0.2 & 0.4 & 0.44 & & 0.19 & 0.19 & 0.31 & 0.24 & 0.44 & 0.48 & 0.41 & 0.73 & 0.26 \\
\hline $\mathrm{U}$ & & 0.032 & & & & & & & & & 0.1 & & 0.1 & & 0.1 & 0.1 & 0.15 & 0.2 & 0.2 & & 0.25 & 0.27 & 0.23 \\
\hline $\mathrm{Eu} / \mathrm{Eu} *$ & 4.31 & 2.14 & 2.22 & 1.78 & 1.54 & 5.57 & 4.33 & 3.79 & 0.93 & 0.94 & 0.8 & 0.97 & 0.94 & 1.0 & 0.91 & 0.96 & 0.86 & 0.96 & 0.96 & 0.91 & 0.90 & 0.91 & 0.99 \\
\hline & & & 0.75 & & 0.95 & & 0.54 & 0.41 & & 0.6 & 0.8 & 0.7 & 1.3 & 0. & 0.6 & 0. & 0.89 & 0. & 1.2 & & 1.3 & 1.36 & 0.71 \\
\hline$(\mathrm{Gd} / \mathrm{Yb}$ & 1.04 & 1.10 & 1.01 & 1.04 & 1.47 & 1.23 & 1.15 & 1.37 & 0.74 & 0.76 & 0.85 & 0.83 & 1.13 & 1.14 & 0.77 & 0.73 & 0.99 & 0.94 & 1.04 & 0.86 & 1.07 & 1.00 & 0.90 \\
\hline & 0.27 & 0.72 & 1.03 & & 1.41 & 0.38 & & 0.99 & & 0.69 & 0.84 & 0.8 & 1. & 1.0 & 0.6 & 0.6 & 0.69 & 1. & 1.1 & 0. & 1.22 & 0.83 & 0.79 \\
\hline$(\mathrm{Sr} / \mathrm{Yb})_{\mathrm{N}}$ & 13.87 & 18.77 & 72.78 & 44.25 & 135 & 19.78 & 19.81 & 20.42 & 1.40 & 1.37 & 2.10 & 0.2 & 0.51 & 2.03 & 1.4 & 1.3 & 0.72 & 2. & 1.11 & 0. & 1.44 & 0.32 & 0.53 \\
\hline$(\mathrm{Ba} / \mathrm{Yb})_{\mathrm{N}}$ & 10.76 & 21.29 & 86.52 & 34.38 & 201 & 405 & 220 & 426 & 0.06 & 0.07 & 0.36 & 0.05 & 0.20 & 0.24 & 0.06 & 0.07 & 0.72 & 2.09 & 1.70 & 1.13 & 1.12 & 0.10 & 7.25 \\
\hline
\end{tabular}

* MG - metagabbro; G-amp - garnet amphibolite; Amp - amphibolite 

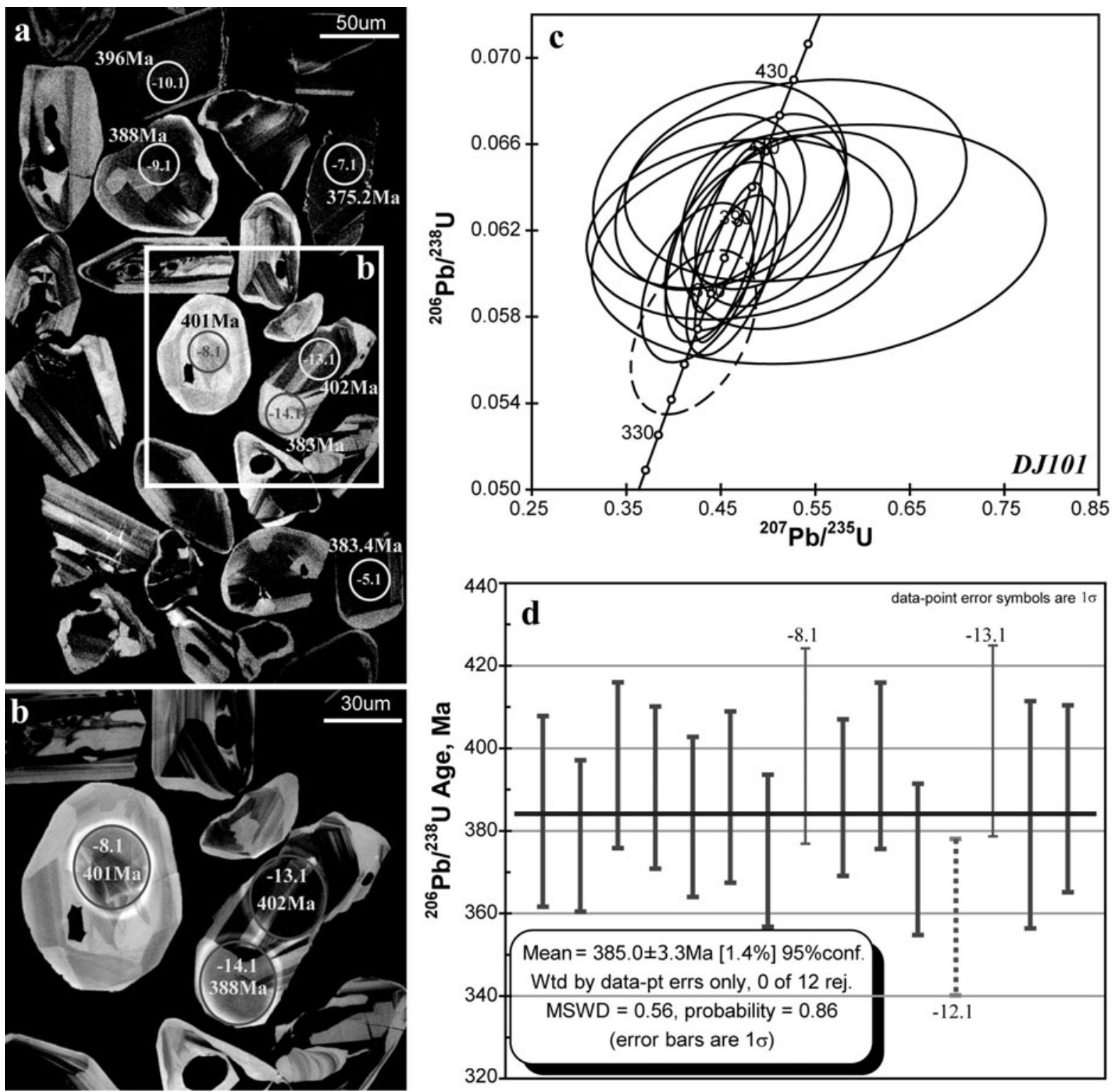

Figure 9. (a, b) Cathodoluminescence (CL) images of zircons separated from metagabbro sample DJ101 (diameter of circle on CL image is $c .30 \mu \mathrm{m}$ ); and (c, d) SHRIMP dating results for zircons in metagabbro.

is highly variable in composition (Supplementary Figure 4 and Supplementary Table 5, both available at http://journals.cambridge.org/geo) and defines the substitution series with magnesiohornblende and tschermakite as end-members. However, amphibole analyses for different samples have similar compositions, suggesting their similar metamorphism conditions from one sample to another.

\section{Geochronology of zircons from metagabbro and amphibolite}

Metagabbro (DJ101) and amphibolite (DJ81) samples collected from the Baikouquan region (Fig. 3b) were used for a zircon chronology study. Most zircons have narrow rims showing a bright CL image (Figs 9a, b, $10 \mathrm{a})$; the narrow rim is generally irregular and discontinuous with a width of $0-20 \mu \mathrm{m}$. The irregular boundary between igneous zircon with oscillatory zoning pattern and its discontinuous rim suggests a replacement reaction. Zircon rims showing a bright $\mathrm{CL}$ pattern are typical of metamorphic zircon (Zheng et al. 2005; Zhang et al. 2007), which differs from hydrothermal zircon rims with dark CL images (Zhu, 2011). Similar metamorphic zircon rims have been reported in most metamorphic rocks, including eclogite and amphibolite.

In the amphibolite igneous zircon would have crystallized during gabbro emplacement, its $\mathrm{U}-\mathrm{Pb}$ age representing magma intrusion time. Most zircon grains in sample DJ101 show a weakly oscillatory zoned pattern combined with a sector zoning pattern. Several zircon grains have narrow metamorphic rims (most $<3 \mu \mathrm{m}$ with bright CL image; Fig. 9a). The narrow metamorphic rim of zircon in the metagabbro likely formed during metamorphism. Zircons in this 

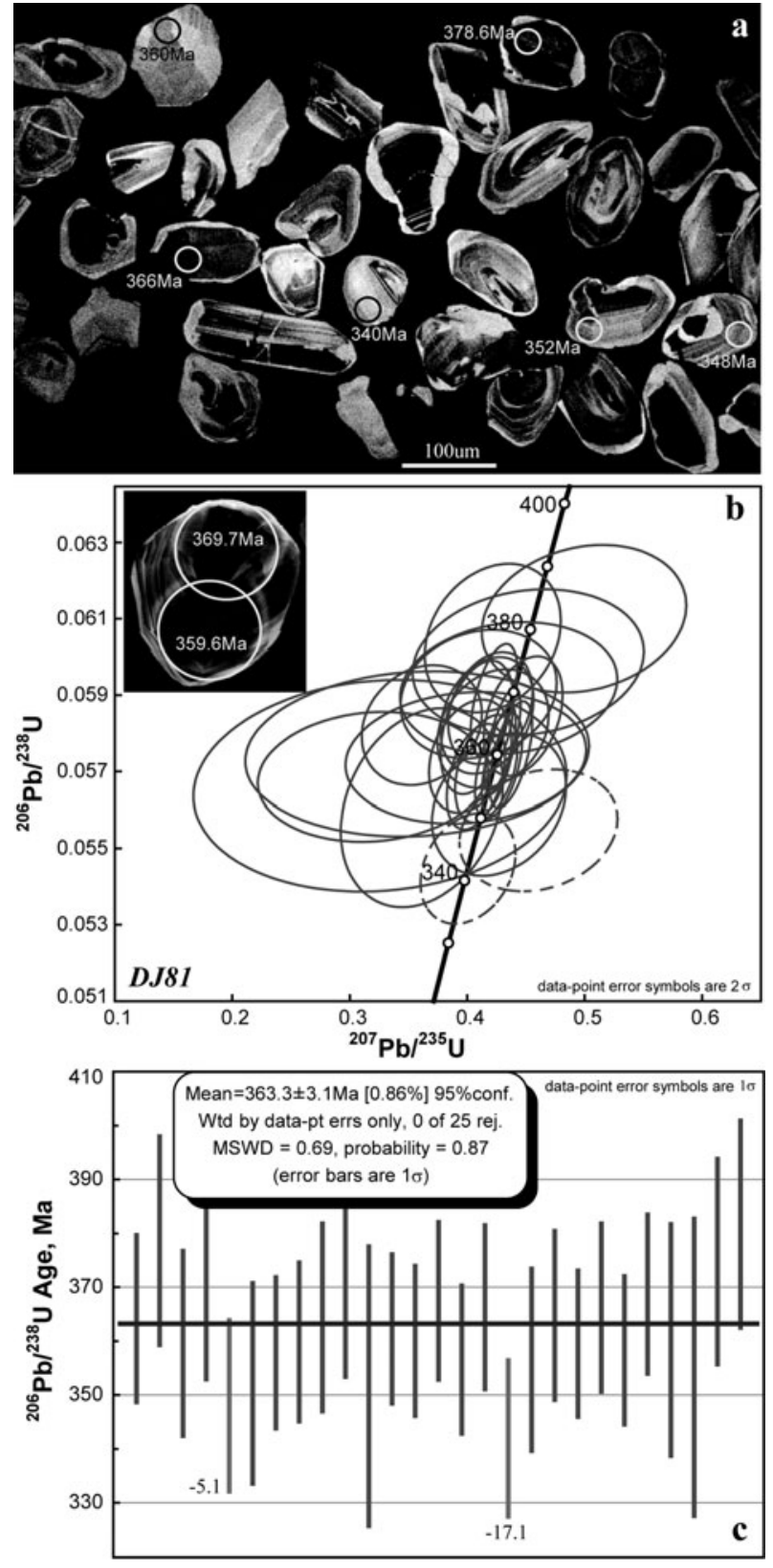

Figure 10. (a) Cathodoluminescence (CL) images of zircons separated from amphibolite sample DJ81; note that most zircons show a narrow rim with bright CL (diameter of circle on CL image is c. $30 \mu \mathrm{m})$; (b, c) SHRIMP dating results for zircons in amphibolite.

metagabbro sample have $\mathrm{Th} / \mathrm{U}$ ratios ranging from 0.24 to 0.84 and apparent $\mathrm{U}-\mathrm{Pb}$ ages of 402-359 Ma. One zircon with an apparent $\mathrm{U}-\mathrm{Pb}$ age of $401 \pm 12 \mathrm{Ma}$ (spot -8.1; Table 1) differs from other zircon grains by its bright CL image (Fig. 9a, b). This may suggest a different origin, and will not be discussed here. Another obviously old apparent $\mathrm{U}-\mathrm{Pb}$ age $(402 \pm 12 \mathrm{Ma}$, spot -13.1$)$ is for a zircon core, while its rim gives a much younger $\mathrm{U}-\mathrm{Pb}$ age $(388 \pm 11 \mathrm{Ma}$, spot -14.1 ; Fig. 9b). These two apparent $\mathrm{U}-\mathrm{Pb}$ ages of $>400 \mathrm{Ma}$ with large errors are not included in the following calculations. Spot -12.1 , with the obviously younger $\mathrm{U}-\mathrm{Pb}$ age (359.1 $\pm 9.5 \mathrm{Ma})$, might represent a mixed $\mathrm{U}-\mathrm{Pb}$ age between igneous zircon and its metamorphic rim, and is also excluded from the following calculation. With the exception of these three problematic spots, all other analyses give a weighted average $\mathrm{U}-\mathrm{Pb}$ age of $385.0 \pm 3.3 \mathrm{Ma}(n=12$, MSWD $=0.56$; Fig. 9 c, d). This age represents magma emplacement prior to metamorphism.

For amphibolite sample DJ81, most zircon grains have narrow metamorphic rims (mostly $<10 \mu \mathrm{m}$, with bright CL image; Fig. 10a). The weakly oscillatory zoned zircon combined with sector zoning pattern is replaced on the rim by a thin metamorphic envelope. It was not possible to obtain a $\mathrm{U}-\mathrm{Pb}$ age for this metamorphosed zircon rim due to its very narrow width (mostly $<5 \mu \mathrm{m}$, rarely up to $20 \mu \mathrm{m}$ ). It was also difficult to avoid the metamorphic zircon rim completely in performing SHRIMP analyses in some cases. For example, one weakly oscillatory zoned zircon (Fig. 10b, inserted CL image) is replaced by a thin metamorphic $\operatorname{rim}(<3 \mu \mathrm{m})$; this weakly oscillatory zoned zircon was dated at $369.7 \mathrm{Ma}$, while another analysis next to this spot produced a younger $\mathrm{U}-\mathrm{Pb}$ age of $359.6 \mathrm{Ma}$. The younger age may have been contaminated by the narrow metamorphic rim.

Zircons have $\mathrm{Th} / \mathrm{U}$ ratios ranging from 0.25 to 0.91 with apparent $\mathrm{U}-\mathrm{Pb}$ ages of 382-342 Ma (Table 1). SHRIMP analyses give a weighted average $\mathrm{U}-\mathrm{Pb}$ age of $363.3 \pm 3.1 \mathrm{Ma}(n=25$, MSWD $=0.69$; Fig. $10 \mathrm{~b}$, c), excluding two spots ( -5.1 and -17.1 with obviously younger ages of $348.0 \pm 7.9 \mathrm{Ma}$ and $341.9 \pm 7.2 \mathrm{Ma}$, respectively). The younger $\mathrm{U}-\mathrm{Pb}$ age could be interpreted as a maximum age of metamorphism. It probably represents a mixture between igneous zircon and a relatively large amount of metamorphic rim. All other analyses may represent mixtures of igneous zircon and the metamorphic rim, with the volume of the metamorphic rim being variable from one spot to another. Thus, the obtained weighted average $\mathrm{U}-\mathrm{Pb}$ age of $363.3 \pm 3.1$ Ma probably does not have an accurate geological meaning as the metamorphic zircon rim could not be excluded completely during SHRIMP analysis.

\section{Geochemistry}

\section{6.a. Trace element geochemistry of clinopyroxene and garnet}

Clinopyroxene in metagabbro (Table 2) is depleted in light REE $\left((\mathrm{Ce} / \mathrm{Yb})_{\mathrm{N}}=0\right.$. 23-0.32; Fig. 11a). Depletions of $\mathrm{Rb}, \mathrm{Ba}, \mathrm{Th}, \mathrm{Ta}$ and $\mathrm{Nb}$ are apparent in primitive-mantle-normalized plot, while $\mathrm{Nd}, \mathrm{Hf}, \mathrm{Ti}$ and heavy REE show enrichments (Fig. 11b). Concentrations of $\mathrm{Ce}, \mathrm{Rb}, \mathrm{Nb}$ and $\mathrm{Pb}$ are highly variable. Clinopyroxene in garnet amphibolite (Table 3) can be divided into two groups based on REE contents (Fig. 11c): one is depleted in light REE or shows a flat REE pattern and another shows a convexupwards chondrite-normalized REE pattern with a weak depletion of light REE and negative Eu anomalies 

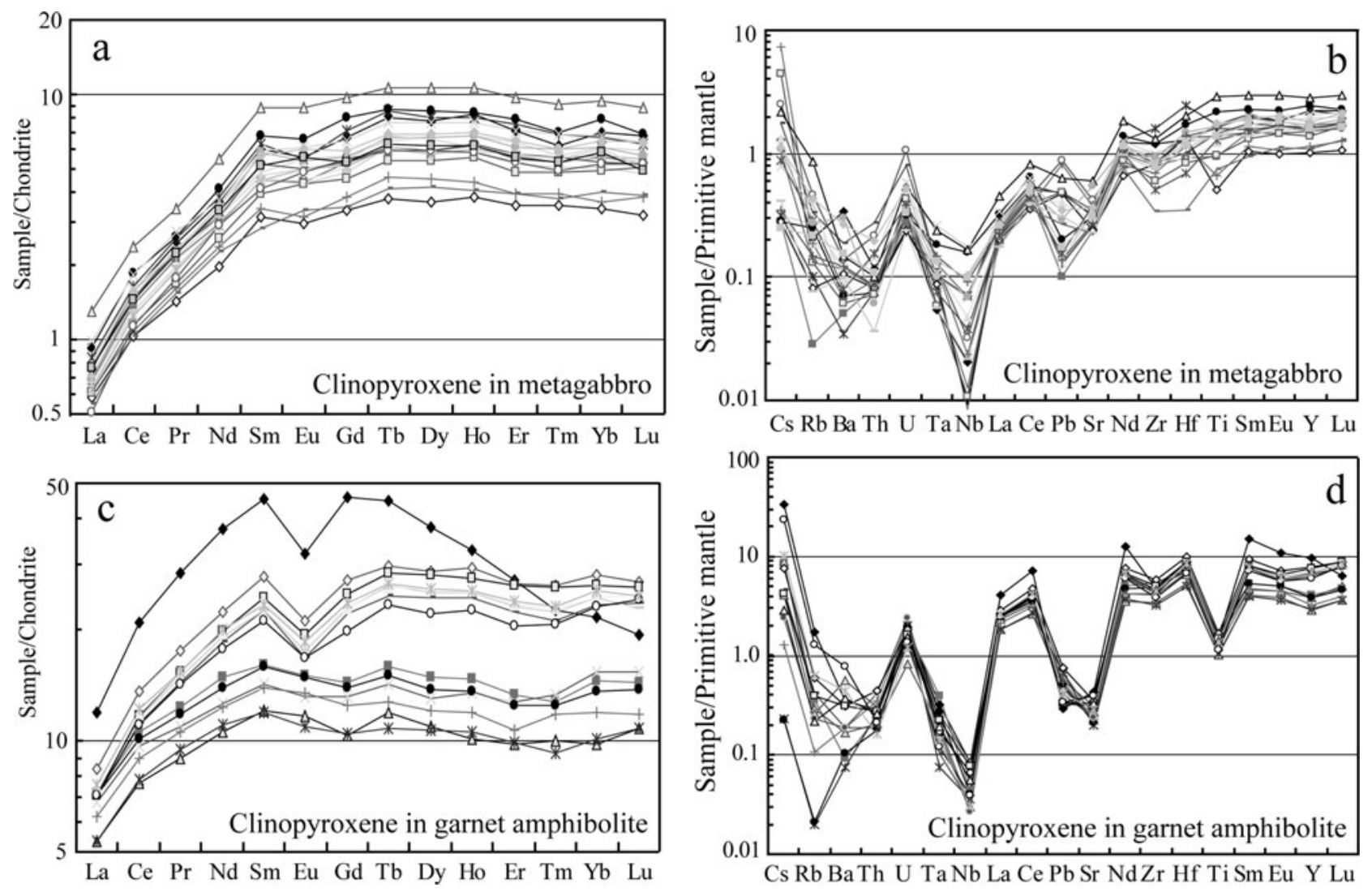

Figure 11. Chondrite-normalized REE distribution patterns for clinopyroxene in (a) metagabbro and (c) garnet amphibolite; primitivemantle-normalized plots for clinopyroxene in (b) metagabbro and (d) garnet amphibolite from the Baikouquan ophioltic mélange. Primitive-mantle data from Sun \& McDonough (1989).

$(\mathrm{Eu} / \mathrm{Eu} *=0.51-0.96)$. Clinopyroxene in garnet amphibolite is characterized by apparent depletions in $\mathrm{Rb}-\mathrm{Ba}, \mathrm{Ta}-\mathrm{Nb}, \mathrm{Pb}-\mathrm{Sr}$ and Ti with highly variable contents of $\mathrm{Cs}, \mathrm{Rb}$ and $\mathrm{Ba}$ (Fig. 11d). Such different geochemical behaviours are consistent with the studied clinopyroxene types; clinopyroxene in metagabbro is magmatic and clinopyroxene in garnet amphibolite is metamorphic in origin.

Garnet in amphibolite is enriched in heavy REE (Table 4) and strongly depleted in light REE relative to chondrite (Fig. 12a). Garnet shows a typical chondritenormalized REE pattern with a strong light REE depletion $\left((\mathrm{Ce} / \mathrm{Yb})_{\mathrm{N}}<0.004\right)$ and variable but mostly insignificant $\mathrm{Eu}$ anomalies. Garnet is strongly depleted in $\mathrm{Ba}, \mathrm{Nb}, \mathrm{La}, \mathrm{Ce}$ and $\mathrm{Sr}$ in a primitive-mantle-normalized plot (Fig. 12b). The contents of $\mathrm{Cs}, \mathrm{U}, \mathrm{Pb}, \mathrm{Nd}, \mathrm{Zr}$ and $\mathrm{Hf}$ in garnet are similar to the primitive-mantle values.

\section{6.b. Geochemistry of metagabbro, amphibolite and lherzolite}

Trace elements of representative samples of metagabbro and amphibolite in the Baikouquan region were measured with ICP-MS (Table 5, Fig. 13). REE contents are very low in metagabbro samples with flat patterns and strongly positive Eu anomalies $(\mathrm{Eu} / \mathrm{Eu} *=$ 1.5-5.6; Fig. 13a). Trace elements are highly variable in primitive-mantle-normalized plots with strong enrichments of $\mathrm{Cs}, \mathrm{Rb}, \mathrm{Ba}$ and $\mathrm{Sr}\left((\mathrm{Ba} / \mathrm{Yb})_{\mathrm{N}}=10.8-426\right.$; Fig. 13b and Table 5). Amphibolite samples from the Baikouquan region show flat REE distribution patterns (Fig. 13c). Most elements (except Ba for some samples; $\left.(\mathrm{Ba} / \mathrm{Yb})_{\mathrm{N}}=0.06-7.25\right)$ show flat patterns in primitivemantle-normalized diagrams, with insignificant depletion of $\mathrm{Ta}, \mathrm{Nb}, \mathrm{Zr}$ and $\mathrm{Hf}$ (high-field-strength elements or HFSE, $(\mathrm{Zr} / \mathrm{Yb})_{\mathrm{N}}=0.67-1.22$; Fig. 13d, Table 5).

Trace elements of representative lherzolite, amphibolite and metagabbro samples collected from the Baijiantan region are listed in Table 6. The REE patterns for metagabbro samples are highly variable with $(\mathrm{Ce} / \mathrm{Yb})_{\mathrm{N}}$ values from 0.64 to 1.76 . Three samples (Klm20, Klm24 and J-129) show similar patterns with strongly positive $\mathrm{Eu}$ anomalies $(\mathrm{Eu} / \mathrm{Eu} *=1.56-2.70$; Fig. 14a); two other samples (Klm28 and Klm37) show similar REE patterns with a slight enrichment in light REE. Enrichments of $\mathrm{Cs}, \mathrm{Rb}, \mathrm{Ba}$ and $\mathrm{Sr}$ are apparent in primitive-mantle-normalized plots $\left((\mathrm{Ba} / \mathrm{Yb})_{\mathrm{N}}=\right.$ $\left.3.1-15 ;(\mathrm{Sr} / \mathrm{Yb})_{\mathrm{N}}=1.3-14\right)$, while other trace elements are highly variable for different samples $\left((\mathrm{Zr} / \mathrm{Yb})_{\mathrm{N}}=\right.$ $0.5-2.0$; Table 6). The flat REE patterns with positive Eu anomalies (Fig. 14a) and insignificant depletion of HFSE relative to the primitive mantle (Fig. 14b) suggest a mid-ocean-ridge basalt (MORB) origin for metagabbro in both the Baijiantan and Baikouquan 

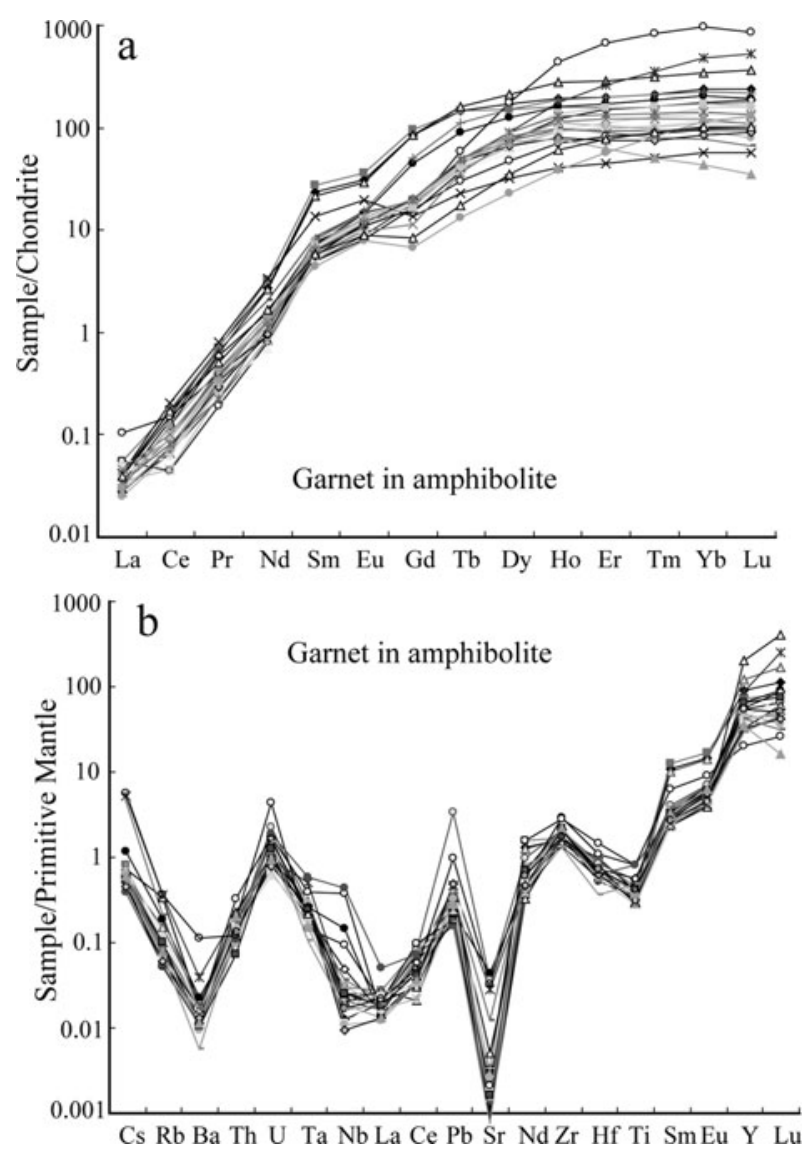

Figure 12. (a) Chondrite-normalized REE distribution patterns and (b) primitive-mantle-normalized plots for garnet in amphibolite. Primitive-mantle data from Sun \& McDonough (1989).

regions. Their different REE contents and Eu anomalies may have been caused by magmatic differentiation and metamorphism. For example, magma differentiation may cause the accumulation of Ca-rich plagioclase, and thus show positive Eu anomalies. Once such gabbro undergoes metamorphism, Ca-rich plagioclase is replaced by zoisite + albite \pm garnet \pm ilmenite \pm chlorite and positive $\mathrm{Eu}$ anomalies will be reduced in the REE distribution pattern due to the decomposition of the Ca-rich phase that is host to most of the Eu. Compared to the Baikouquan metagabbro, metagabbro in the Baijiantan region is characterized by a higher content of REE, Zr, Hf and Y (Fig. 14b). The amphibolite sample Klm27 shows a flat REE pattern (Fig. 14c) and a primitive-mantle-normalized plot (Fig. 14d) shows a flat pattern with slight depletion of Th and Ta. The flat REE patterns and insignificant depletions of HFSE imply that both metagabbro and amphibolite originated from MORB-type magmatism. Clinopyroxene in metagabbro still shows a magmatic signature, even though it was partly replaced by amphibole.

Lherzolite samples have similar REE distribution patterns, showing obvious depletion of light REE without Eu anomalies (Fig. 15a). Total REE contents in these samples vary from $1.89 \mathrm{ppm}$ to $3.71 \mathrm{ppm}$ with $(\mathrm{Ce} / \mathrm{Yb})_{\mathrm{N}}$ of $0.11-0.75$. The $\mathrm{Cs}, \mathrm{Rb}, \mathrm{Ba}$, Th and Sr en- richments are apparent in primitive-mantle-normalized plots (Fig. 15b); the U, La, Ce and $\mathrm{Nd}$ contents are highly variable with obvious depletions of $\mathrm{La}, \mathrm{Ce}$ and Nd.

The $\mathrm{Sr}-\mathrm{Nd}$ isotopic compositions of representative lherzolite and metagabbro samples are listed in Table 7. Four lherzolite samples define one isochron, which gives an isochron age of $474 \pm 38 \mathrm{Ma}$ (MSWD $=0.18$; Fig. 16a) with $\varepsilon_{\mathrm{Nd}(t)}$ of +8.9 . One $\mathrm{Rb}-\mathrm{Sr}$ isochron for metagabbro samples in the Baijiantan region indicates an isochron age of $335 \pm 10 \mathrm{Ma}$ (MSWD = 1.4; Fig. 16b) with an initial ${ }^{87} \mathrm{Sr} /{ }^{86} \mathrm{Sr}$ ratio of $0.704597 \pm 0.000011$. These metagabbro samples have positive $\varepsilon_{\mathrm{Nd}(t)}$ values of +5.9 to +11.0 and initial ${ }^{87} \mathrm{Sr} /{ }^{86} \mathrm{Sr}$ ratios of $0.70447-0.70458$. Seven metagabbro samples collected from the Baikouquan region give an $\mathrm{Sm}-\mathrm{Nd}$ isochron age of $379 \pm 31 \mathrm{Ma}$ (MSWD $=0.3$; Fig. 16c), with $\varepsilon_{\mathrm{Nd}(t)}$ of +9.4 . These metagabbro samples have similar $\varepsilon_{\mathrm{Nd}(t)}$ values $(+9.3$ to +9.5$)$ and initial ${ }^{87} \mathrm{Sr} /{ }^{86} \mathrm{Sr}$ ratios of $0.7045-0.7054$. Six $\mathrm{Rb}-$ $\mathrm{Sr}$ isotopic analyses (except for sample BK13) form one isochron which provides an age of $333 \pm 12 \mathrm{Ma}$ (MSWD = 4.0; Fig. 16d) with an initial ${ }^{87} \mathrm{Sr} /{ }^{86} \mathrm{Sr}$ ratio of $0.705336 \pm 0.000032$. The $\mathrm{Sm}-\mathrm{Nd}$ isotopic isochron age (379 Ma) of metagabbro samples in the Baikouquan region likely represents a magmatic event. Their $\mathrm{Rb}-\mathrm{Sr}$ isochron age (333 Ma) may record a metamorphic event, as the $\mathrm{Rb}-\mathrm{Sr}$ isotopic system can be easily reset while that of the $\mathrm{Sm}-\mathrm{Nd}$ isotopic system may remain stable during metamorphism.

Lherzolite samples are characterized by high positive $\varepsilon_{\mathrm{Nd}(t)}$ and low initial ${ }^{87} \mathrm{Sr} /{ }^{86} \mathrm{Sr}$ values, different from the metagabbro samples which have higher initial ${ }^{87} \mathrm{Sr} /{ }^{86} \mathrm{Sr}$ ratios (Supplementary Figure 5, available at http://journals.cambridge.org/geo). The lherzolite samples with $\varepsilon_{\mathrm{Nd}(t)}$ values of +8.8 to +9.1 and initial ${ }^{87} \mathrm{Sr} /{ }^{86} \mathrm{Sr}$ ratios of $0.7037-0.7040$ are rather homogeneous in $\mathrm{Sr}-\mathrm{Nd}$ isotopic compositions, whereas metagabbro samples have a relatively wide $\mathrm{Sr}-\mathrm{Nd}$ isotopic compositional range; the $\varepsilon_{\mathrm{Nd}(t)}$ values fall within a small range $(+9.3$ to +11.0$)$ except for sample $\mathrm{K} \operatorname{lm} 24$, which has a $\varepsilon_{\mathrm{Nd}(t)}$ value of +5 .9. High positive $\varepsilon_{\mathrm{Nd}(t)}$ values for metagabbro samples suggest a depleted mantle origin, while their high initial ${ }^{87} \mathrm{Sr} /{ }^{86} \mathrm{Sr}$ ratios suggest interaction with $\mathrm{Sr}$ sourced from continental material or marine water during metamorphism.

\section{Discussion}

\section{7.a. Metamorphic $\boldsymbol{P}-\boldsymbol{T}$ conditions for garnet amphibolite}

We used the PERPLE_X computer software package (Connolly, 2005; Massonne, 2009) to calculate the phase relations of garnet amphibolite. The thermodynamic dataset of Holland \& Powell (1998; updated in 2002) for numerous mineral end-members (file hp02ver.dat) was applied. The following solid-solution series, which are compatible with the above dataset, were selected from this file: amphibole (GlTrTsPg, a mixture of glaucophane, tremolite, tschermakite, 

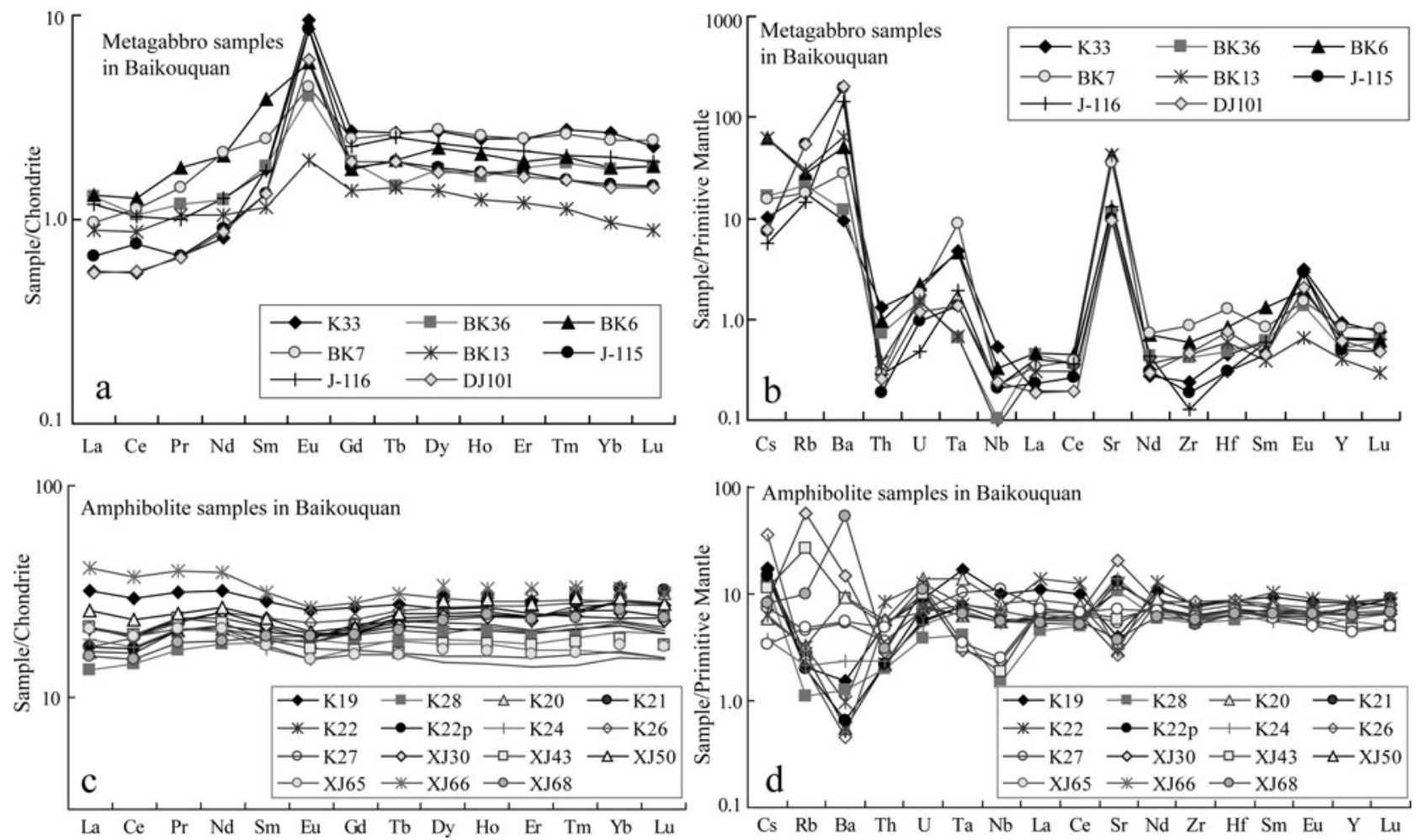

Figure 13. Trace element plots for (a, b) metagabbro and (c, d) amphibolite samples collected from the Baikouquan region. Chondrite and primitive-mantle data from Sun \& McDonough (1989).

pargasite and corresponding $\mathrm{Fe}^{2+}$ end-members); biotite (Bio(HP): phlogopite, eastonite, corresponding $\mathrm{Fe}^{2+}$ and $\mathrm{Mn}^{2+}$ end-members); clinopyroxene (Omph(HP): diopside, hedenbergite and jadeite); garnet (Gt(HP): almandine, grossular, pyrope and spessartine); and ilmenite (IlGkPy: ideal mixture of ilmenite, geikielite and pyrophanite end-members).

Thermodynamic calculations for garnet amphibolite (sample K26, for its composition see Supplementary Table 6 available at http://journals.cambridge.org/geo) were started at $400^{\circ} \mathrm{C}$ and $8 \mathrm{kbar}$ (Fig. 17a). Plagioclase does not occur in this $P-T$ pseudosection whereas garnet and amphibole are present at all $P_{-}$ $T$ conditions; quartz occurs only at low temperature $\left(<510^{\circ} \mathrm{C}\right)$. The mineral phases in the studied samples include amphibole, garnet, zoisite, chlorite, clinopyroxene, biotite, ilmenite, sphene, rutile and quartz. Quartz, biotite and rutile mostly occur as inclusions in garnet (Fig. 7b-g). This mineral assemblage is consistent with evolution from F1 to F2 in the $P-T$ pseudosection (Fig. 17a), where the mineral assemblage of biotite-chlorite-clinopyroxeneepidote-amphibole-garnet-sphene-quartz (F1) transformed to the mineral assemblage of biotite-chloriteepidote-amphibole-garnet-sphene-quartz- $\mathrm{H}_{2} \mathrm{O}$ (F2) with isobaric temperature increase, during which period clinopyroxene disappeared and an $\mathrm{H}_{2} \mathrm{O}$ phase formed. Rutile growth started with a further temperature increase at F3 and formed a mineral assemblage of biotite-chlorite-epidote-amphibolegarnet-sphene-rutile- $\mathrm{H}_{2} \mathrm{O}$ without quartz. We sug- gest that the metamorphism recorded in sample K26 reached the $P-T$ conditions of F3 (c. $15 \mathrm{kbar}, 520$ $560^{\circ} \mathrm{C}$ ).

The $P-T$ path for garnet amphibolite started from F1 to F2, entered F3 isobarically and finally reached the amphibolite grade (F4; Fig. 17b) with pressure decrease. The $P-T$ environment of stage $\mathrm{F} 4$ is based on the amphibole composition, estimated using the program developed by Gerya et al. (1997); amphibole analyses are listed in Supplementary Table 5 (available at http://journals.cambridge.org/geo). The $P-T$ values based on amphibole compositions of sample K26 are 566- $633^{\circ} \mathrm{C}$ and $4-6 \mathrm{kbar}$; sample $\mathrm{K} 20$ corresponds to $583-630^{\circ} \mathrm{C}$ and $4-6 \mathrm{kbar}$; sample $\mathrm{K} 21$ is $580-656^{\circ} \mathrm{C}$ (4.2-7.7 kbar); and sample $\mathrm{K} 24$ is $580-645^{\circ} \mathrm{C}$ (4$7 \mathrm{kbar}$ ). The overlap of these estimates determined the $P-T$ environment of $\mathrm{F} 4$.

The mineral assemblage of garnet amphibolite records progressive metamorphism (from F1 to F3) which was followed by retrograde metamorphism (from F3 to F4). The $P-T$ path could therefore be clockwise (Fig. 17b), reflecting a subduction condition in which gabbro (the original rock of the studied amphibolite) was first subducted and then exhumed.

\section{7.b. Age constraints and tectonic implications}

Zircon SHRIMP analyses give a weighted average $\mathrm{U}-\mathrm{Pb}$ age of $c .385 \mathrm{Ma}$ for metagabbro (Fig. 9) and $363 \mathrm{Ma}$ for amphibolite (Fig. 10). The $\mathrm{Sm}-\mathrm{Nd}$ isotopic analyses of metagabbro samples indicate an isochron 
Table 6. Trace element contents of lherzolite, amphibolite and metagabbro in Baijiantan ophiolitic mélange (in ppm).

\begin{tabular}{|c|c|c|c|c|c|c|c|c|c|c|c|c|}
\hline $\begin{array}{l}\text { Sample } \\
\text { Rock }\end{array}$ & $\begin{array}{c}\text { Klm23 } \\
\text { Lherzolite }\end{array}$ & $\begin{array}{c}\text { Klm30 } \\
\text { Lherzolite }\end{array}$ & $\begin{array}{c}\text { Klm31 } \\
\text { Lherzolite }\end{array}$ & $\begin{array}{c}\text { Klm32 } \\
\text { Lherzolite }\end{array}$ & $\begin{array}{c}\text { Klm33 } \\
\text { Lherzolite }\end{array}$ & $\begin{array}{c}\text { Klm34 } \\
\text { Lherzolite }\end{array}$ & $\begin{array}{c}\text { Klm27 } \\
\text { Amphibolite }\end{array}$ & $\begin{array}{c}\text { Klm20 } \\
\text { Metagabbro }\end{array}$ & $\begin{array}{c}\text { Klm24 } \\
\text { Metagabbro }\end{array}$ & $\begin{array}{c}\text { Klm28 } \\
\text { Metagabbro }\end{array}$ & $\begin{array}{c}\text { Klm37 } \\
\text { Metagabbro }\end{array}$ & $\begin{array}{c}\text { J-129 } \\
\text { Metagabbro }\end{array}$ \\
\hline $\mathrm{Li}$ & 1.72 & 2.22 & 2.28 & 1.16 & 1.53 & 1.62 & 2.17 & 10.21 & 18.9 & 6.50 & 6.56 & 25.0 \\
\hline $\mathrm{Be}$ & 0.23 & 0.17 & 0.17 & 0.19 & 0.23 & 0.18 & 0.41 & 0.24 & 0.19 & 0.50 & 0.53 & 0.15 \\
\hline $\mathrm{Sc}$ & 77.0 & 14.4 & 14.9 & 9.2 & 13.0 & 13.0 & 40.5 & 54.9 & 35.7 & 30.6 & 31.3 & 50.4 \\
\hline V & 185 & 91.2 & 85.4 & 58.9 & 78.1 & 79.1 & 255 & 159 & 117 & 200 & 200 & 340 \\
\hline $\mathrm{Cr}$ & 1100 & 2412 & 2295 & 2252 & 2358 & 2449 & 265 & 514 & 185 & 591 & 648 & 213 \\
\hline Co & 60.1 & 93.0 & 93.9 & 97.9 & 99.7 & 100.6 & 39.1 & 48.0 & 24.5 & 38.5 & 38.5 & 18.0 \\
\hline $\mathrm{Ni}$ & 208 & 1849 & 1892 & 1999 & 2003 & 1978 & 70.7 & 134 & 57.6 & 302 & 310 & 73.1 \\
\hline $\mathrm{Cu}$ & 5.1 & 24.4 & 23.9 & 11.3 & 14.5 & 14.8 & 34.2 & 110.5 & 1.99 & 32.6 & 33.4 & 0.98 \\
\hline $\mathrm{Ga}$ & 4.42 & 2.75 & 2.83 & 2.09 & 2.90 & 2.80 & 14.69 & 7.82 & 8.43 & 12.27 & 12.30 & 9.87 \\
\hline $\mathrm{Rb}$ & 0.001 & 1.47 & 0.62 & 1.23 & 0.96 & 0.85 & 4.17 & 3.23 & 11.31 & 6.17 & 5.59 & 36.10 \\
\hline $\mathrm{Sr}$ & 72.82 & 26.53 & 24.51 & 21.94 & 24.25 & 26.85 & 251 & 181 & 480 & 81.13 & 86.06 & 1454 \\
\hline Y & 3.51 & 4.49 & 4.58 & 2.84 & 4.56 & 4.54 & 31.84 & 5.80 & 11.10 & 16.49 & 16.44 & 19.30 \\
\hline $\mathrm{Zr}$ & 5.77 & 5.92 & 5.81 & 6.21 & 6.75 & 6.61 & 88.32 & 5.21 & 11.8 & 66.00 & 61.24 & 54.5 \\
\hline $\mathrm{Nb}$ & 0.59 & 1.03 & 0.99 & 1.01 & 1.08 & 0.99 & 2.26 & 0.51 & 0.28 & 2.24 & 2.17 & 2.52 \\
\hline $\mathrm{Cs}$ & 0.12 & 0.12 & 0.13 & 0.10 & 0.11 & 0.11 & 0.19 & 0.24 & 0.33 & 0.32 & 0.33 & 0.98 \\
\hline $\mathrm{Ba}$ & 8.29 & 25.42 & 25.28 & 6.92 & 7.74 & 9.05 & 82.70 & 44.06 & 221 & 64.41 & 65.63 & 516 \\
\hline $\mathrm{La}$ & 0.28 & 0.13 & 0.11 & 0.13 & 0.23 & 0.24 & 3.38 & 0.64 & 1.04 & 4.25 & 4.03 & 1.82 \\
\hline $\mathrm{Ce}$ & 0.67 & 0.16 & 0.17 & 0.32 & 0.57 & 0.54 & 8.61 & 1.29 & 2.97 & 9.40 & 9.16 & 5.59 \\
\hline Pr & 0.088 & 0.020 & 0.020 & 0.045 & 0.092 & 0.084 & 1.56 & 0.18 & 0.55 & 1.59 & 1.58 & 1.00 \\
\hline $\mathrm{Nd}$ & 0.42 & 0.16 & 0.16 & 0.25 & 0.51 & 0.51 & 7.91 & 0.79 & 3.25 & 7.58 & 7.26 & 5.48 \\
\hline $\mathrm{Sm}$ & 0.18 & 0.14 & 0.13 & 0.11 & 0.20 & 0.21 & 2.69 & 0.35 & 1.19 & 2.14 & 2.05 & 1.95 \\
\hline $\mathrm{Eu}$ & 0.083 & 0.077 & 0.076 & 0.048 & 0.091 & 0.093 & 1.04 & 0.25 & 0.89 & 0.69 & 0.67 & 2.21 \\
\hline $\mathrm{Gd}$ & 0.33 & 0.34 & 0.35 & 0.20 & 0.39 & 0.39 & 3.84 & 0.68 & 1.91 & 2.43 & 2.45 & 3.18 \\
\hline $\mathrm{Tb}$ & 0.062 & 0.070 & 0.073 & 0.038 & 0.076 & 0.075 & 0.72 & 0.13 & 0.34 & 0.41 & 0.42 & 0.57 \\
\hline Dy & 0.40 & 0.52 & 0.54 & 0.26 & 0.54 & 0.54 & 4.98 & 0.84 & 2.07 & 2.67 & 2.67 & 3.38 \\
\hline Ho & 0.089 & 0.12 & 0.12 & 0.062 & 0.13 & 0.13 & 1.09 & 0.18 & 0.45 & 0.55 & 0.55 & 0.78 \\
\hline $\mathrm{Er}$ & 0.26 & 0.37 & 0.38 & 0.18 & 0.38 & 0.37 & 3.16 & 0.50 & 1.32 & 1.54 & 1.56 & 2.29 \\
\hline $\mathrm{Tm}$ & 0.038 & 0.059 & 0.059 & 0.029 & 0.059 & 0.057 & 0.49 & 0.073 & 0.21 & 0.24 & 0.23 & 0.40 \\
\hline $\mathrm{Yb}$ & 0.25 & 0.40 & 0.39 & 0.19 & 0.38 & 0.37 & 3.04 & 0.46 & 1.27 & 1.48 & 1.47 & 2.43 \\
\hline $\mathrm{Lu}$ & 0.038 & 0.060 & 0.062 & 0.027 & 0.056 & 0.055 & 0.46 & 0.068 & 0.20 & 0.22 & 0.21 & 0.40 \\
\hline $\mathrm{Hf}$ & 0.18 & 0.19 & 0.17 & 0.16 & 0.18 & 0.17 & 2.51 & 0.19 & 0.48 & 1.64 & 1.44 & 1.83 \\
\hline $\mathrm{Ta}$ & 0.011 & 0.016 & 0.014 & 0.012 & 0.014 & 0.015 & 0.09 & 0.013 & 0.05 & 0.060 & 0.045 & 0.31 \\
\hline Th & 0.13 & 0.12 & 0.12 & 0.13 & 0.12 & 0.12 & 0.17 & 0.12 & 0.06 & 0.46 & 0.45 & 0.41 \\
\hline U & 0.058 & 0.040 & 0.031 & 0.007 & 0.013 & 0.023 & 0.04 & 0.063 & 0.02 & 0.12 & 0.12 & 0.10 \\
\hline $\mathrm{Eu} / \mathrm{Eu} *$ & 1.04 & 1.09 & 1.07 & 1.00 & 0.99 & 1.01 & 0.99 & 1.56 & 1.79 & 0.92 & 0.91 & 2.70 \\
\hline$(\mathrm{Ce} / \mathrm{Yb})_{\mathrm{N}}$ & 0.75 & 0.11 & 0.12 & 0.46 & 0.42 & 0.41 & 0.79 & 0.78 & 0.65 & 1.76 & 1.73 & 0.64 \\
\hline$(\mathrm{Gd} / \mathrm{Yb})_{\mathrm{N}}$ & 1.10 & 0.71 & 0.73 & 0.86 & 0.85 & 0.87 & 1.04 & 1.22 & 1.25 & 1.36 & 1.38 & 1.08 \\
\hline$(\mathrm{Zr} / \mathrm{Yb})_{\mathrm{N}}$ & 1.02 & 0.66 & 0.65 & 1.42 & 0.79 & 0.79 & 1.28 & 0.50 & 0.41 & 1.96 & 1.84 & 0.99 \\
\hline$(\mathrm{Sr} / \mathrm{Yb})_{\mathrm{N}}$ & 6.85 & 1.57 & 1.46 & 2.67 & 1.50 & 1.71 & 1.93 & 9.23 & 8.85 & 1.28 & 1.37 & 14.00 \\
\hline$(\mathrm{Ba} / \mathrm{Yb})_{\mathrm{N}}$ & 2.36 & 4.53 & 4.53 & 2.54 & 1.45 & 1.74 & 1.92 & 6.79 & 12.31 & 3.07 & 3.15 & 15.00 \\
\hline
\end{tabular}



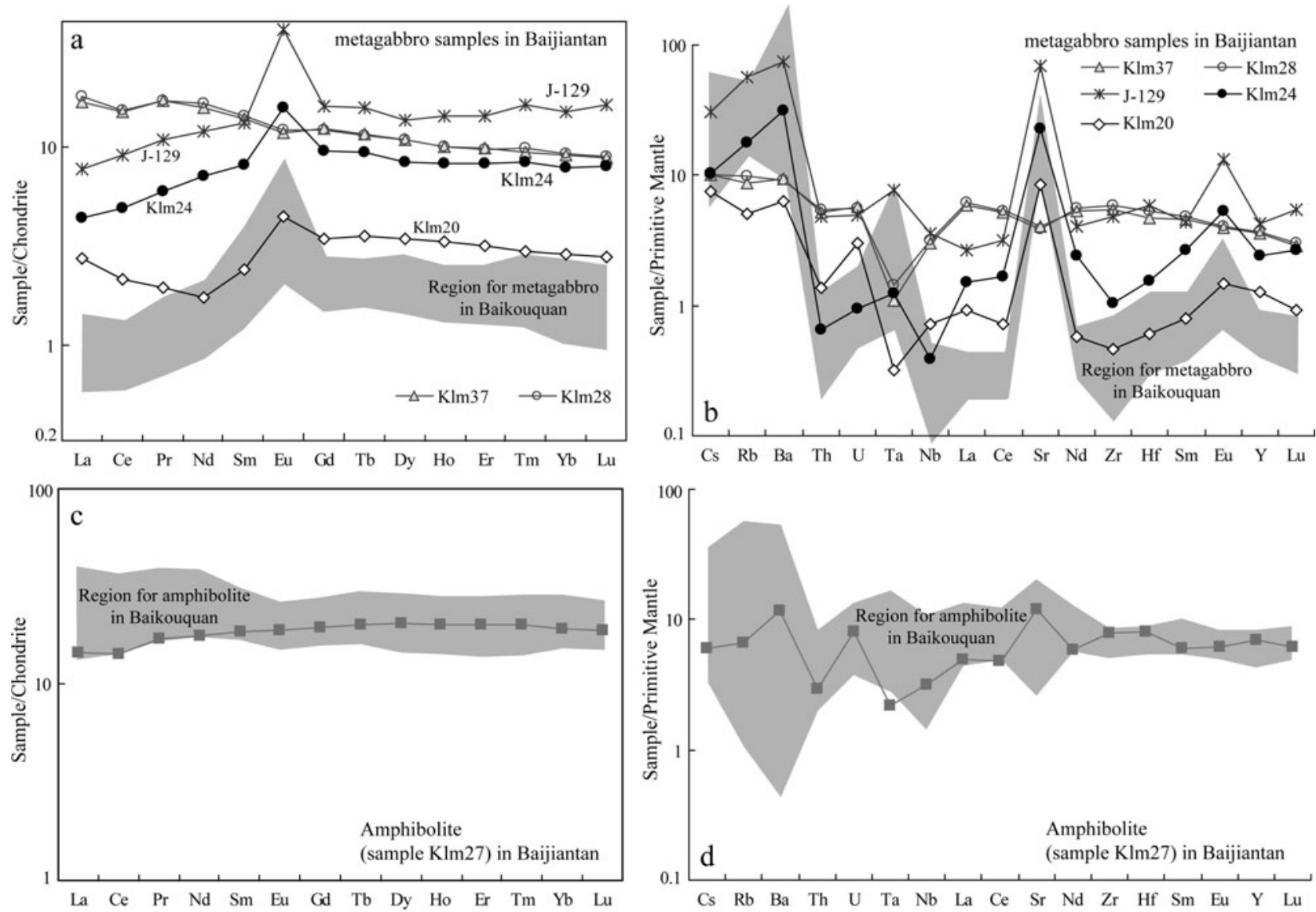

Figure 14. Trace element plots for (a, b) metagabbro and (c, d) amphibolite samples collected from the Baijiantan region. The shaded regions represent the corresponding variations of trace elements in the Baikouquan region. Chondrite and primitive-mantle data from Sun \& McDonough (1989).
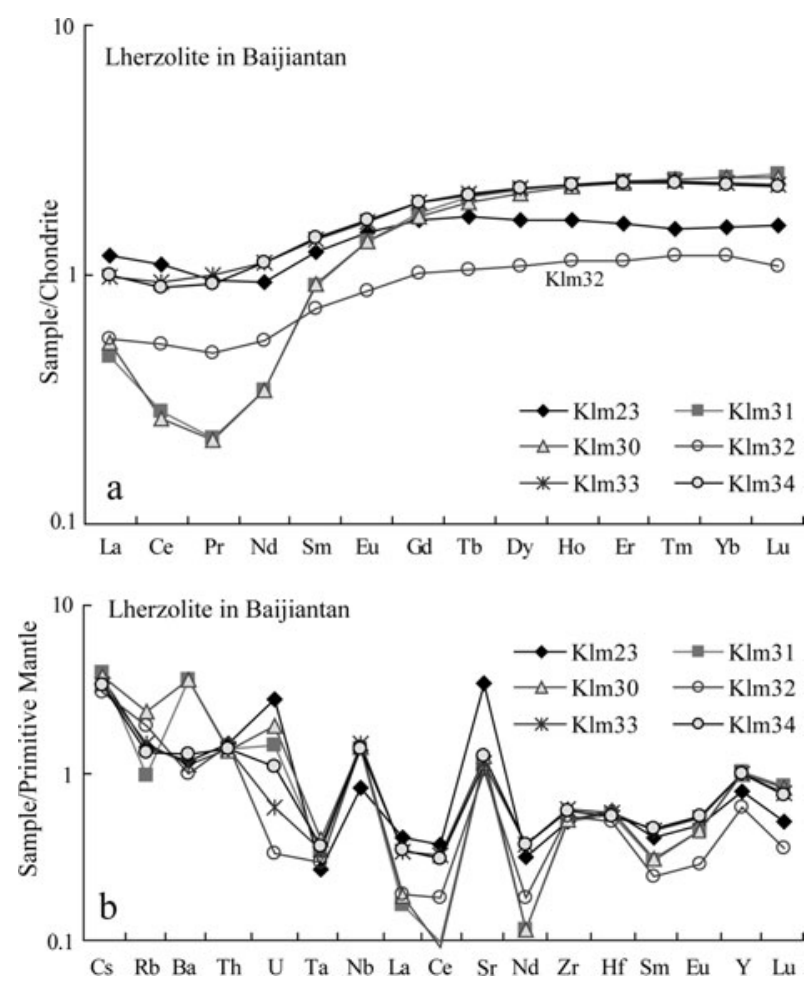

Figure 15. (a) Chondrite-normalized REE distribution patterns and (b) primitive-mantle-normalized plot for lherzolite. Primitive-mantle data from Sun \& McDonough (1989). age of $379 \mathrm{Ma}$ (Fig. 16c). We consider that the U$\mathrm{Pb}$ age of $385 \mathrm{Ma}$ represents magma intrusion time, whereas the other two ages may be affected by metamorphism. The youngest $\mathrm{U}-\mathrm{Pb}$ age $(342 \mathrm{Ma}$; spot 17.1 for amphibolite sample DJ81; Table 1) probably corresponds to the peak metamorphic stage (F3). Retrograde metamorphism reached the $P-T$ environment of F4 at c. $333 \mathrm{Ma}$ based on the $\mathrm{Rb}-\mathrm{Sr}$ isotopic isochron (Fig. 16b, d). This implies that the gabbro in the Junggar oceanic floor was subducted, underwent metamorphism in a subduction zone (at $c .342 \mathrm{Ma}$ ) and was exhumed to shallow depths (c. $5 \mathrm{kbar})$ at $c .333 \mathrm{Ma}$ (Fig. 17c).

The Sm-Nd isochron age of $474 \mathrm{Ma}$ (Fig. 16a) might record a partial melting event of depleted mantle that produced MORB-type magma and formed oceanic floor. This age is consistent with the microfossils separated from abyssal radiolarian chert in the Baijiantan region, which indicates that the palaeo-ocean closed during Late Ordovician time (He et al. 2007). The blueschist lenses in the Tangbale ophiolite mélange were dated at 458-470 Ma (Zhang, 1997). The similarity in ages as well as in the geological records of these ophiolitic mélanges (Feng, 1986; Zhang \& Huang, 1992; Zhu et al. 2008) support the suggestion that the Tangbale ophiolite mélange was connected with the Baijiantan-Baikouquan ophiolitic mélanges 
Table 7. Sr-Nd isotopic compositions for representative lherzolite and metagabbro in the Baijiantan and Baikouquan ophiolitic mélanges.

\begin{tabular}{|c|c|c|c|c|c|c|c|c|c|}
\hline & ${ }^{87} \mathrm{Rb} /{ }^{86} \mathrm{Sr}$ & ${ }^{87} \mathrm{Sr} /{ }^{86} \mathrm{Sr}$ & $2 \sigma$ & $\mathrm{Sr}_{\mathrm{i}(t)}$ & ${ }^{143} \mathrm{Sm} /{ }^{144} \mathrm{Nd}$ & ${ }^{143} \mathrm{Nd} /{ }^{144} \mathrm{Nd}$ & $2 \sigma$ & $\varepsilon_{\mathrm{Nd}(t)}$ & $t_{\mathrm{DM}}(\mathrm{Ma})$ \\
\hline \multicolumn{10}{|c|}{ Baijiantan lherzolite $(t=470 \mathrm{Ma})$} \\
\hline $\mathrm{K} \operatorname{lm} 23$ & 0.0280 & 0.704078 & 0.000013 & 0.703890 & 0.2012 & 0.513110 & 0.000019 & 8.9 & 501 \\
\hline $\mathrm{K} \operatorname{lm} 31$ & 0.0831 & 0.704210 & 0.000015 & 0.703654 & 0.1743 & 0.513019 & 0.000015 & 8.8 & 511 \\
\hline $\mathrm{K} \operatorname{lm} 32$ & 0.0068 & 0.704017 & 0.000012 & 0.703972 & 0.1941 & 0.513090 & 0.000017 & 9.0 & 475 \\
\hline $\mathrm{K} \operatorname{lm} 33$ & 0.0173 & 0.704071 & 0.000011 & 0.703954 & 0.2648 & 0.513314 & 0.000018 & 9.1 & 487 \\
\hline \multicolumn{10}{|c|}{ Baijiantan metagabbro $(t=380 \mathrm{Ma})$} \\
\hline $\mathrm{K} \operatorname{lm} 20$ & 0.0318 & 0.704753 & 0.000011 & 0.704581 & 0.2366 & 0.513302 & 0.000020 & 11.0 & 1003 \\
\hline $\mathrm{K} \operatorname{lm} 24$ & 0.1988 & 0.705547 & 0.000013 & 0.704471 & 0.1858 & 0.512915 & 0.000013 & 5.9 & 1287 \\
\hline $\mathrm{K} 1 \mathrm{~m} 28$ & 0.0441 & 0.704802 & 0.000012 & 0.704563 & 0.2236 & 0.513202 & 0.000017 & 9.7 & 786 \\
\hline \multicolumn{10}{|c|}{ Baikouquan metagabbro $(t=380 \mathrm{Ma})$} \\
\hline $\mathrm{Bk} 7$ & 0.0534 & 0.705662 & 0.000016 & 0.705373 & 0.2509 & 0.513248 & 0.000011 & 9.3 & 398 \\
\hline Bk13 & 0.1716 & 0.705468 & 0.000012 & 0.704540 & 0.2570 & 0.513273 & 0.000013 & 9.5 & 430 \\
\hline Bk36 & 0.0616 & 0.705636 & 0.000014 & 0.705303 & 0.2295 & 0.513198 & 0.000014 & 9.3 & 454 \\
\hline DJ101 & 0.5798 & 0.707687 & 0.000027 & 0.704550 & 0.3005 & 0.513373 & 0.000014 & 9.3 & 391 \\
\hline K33 & 0.1201 & 0.705443 & 0.000019 & 0.704793 & 0.2892 & 0.513347 & 0.000014 & 9.4 & 396 \\
\hline $\mathrm{J} 115$ & 0.0830 & 0.705711 & 0.000014 & 0.705262 & 0.2895 & 0.513349 & 0.000015 & 9.4 & 399 \\
\hline J116 & 0.2871 & 0.706727 & 0.000021 & 0.705174 & 0.2436 & 0.513235 & 0.000012 & 9.4 & 429 \\
\hline
\end{tabular}
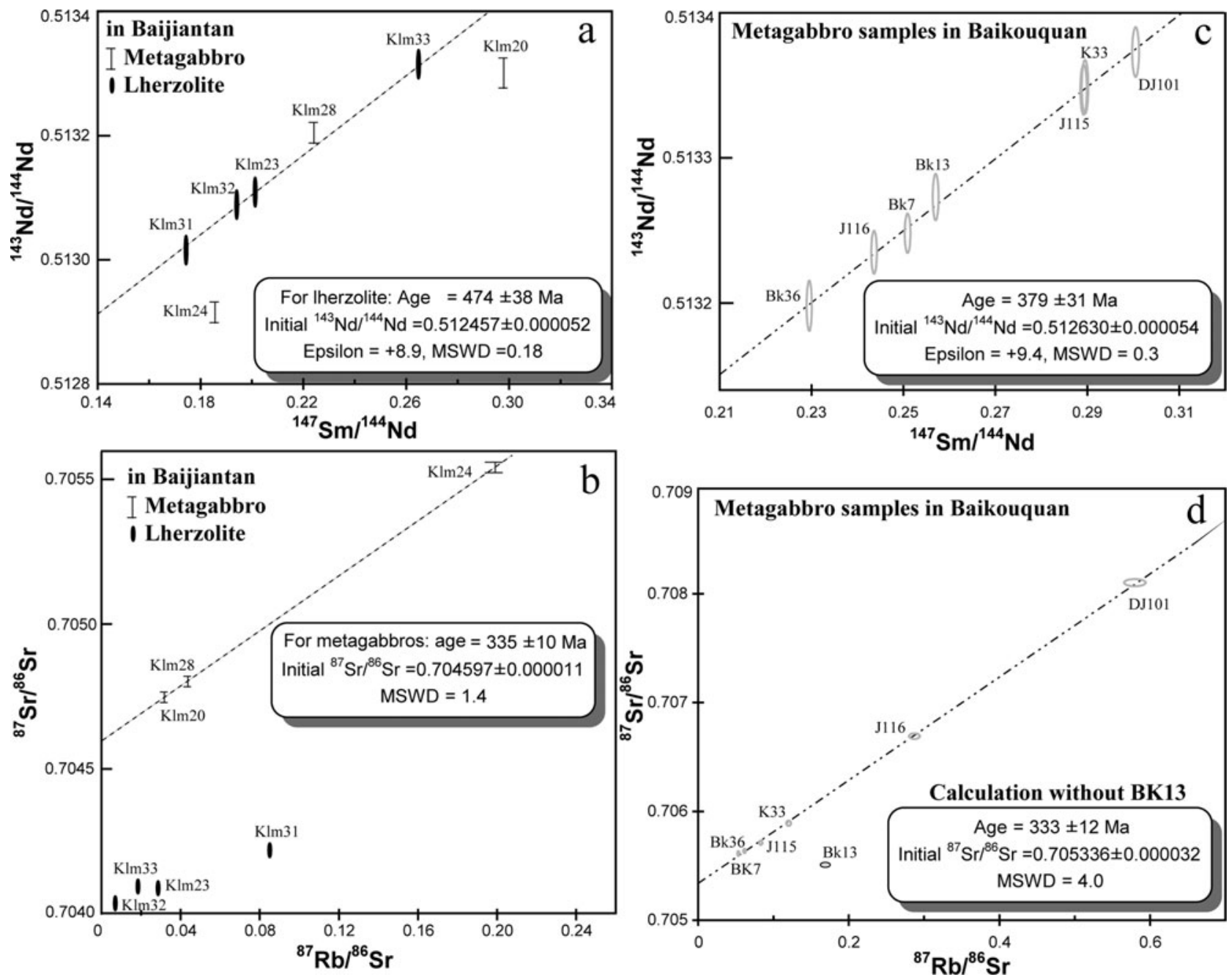

Figure 16. (a) Isochron plot of ${ }^{147} \mathrm{Sm} /{ }^{144} \mathrm{Nd} v .{ }^{143} \mathrm{Nd} /{ }^{144} \mathrm{Nd}$ for lherzolite samples in the Baijiantan region with an age of $474 \mathrm{Ma}$ (metagabbro samples included for comparison). (b) $\mathrm{Rb}-\mathrm{Sr}$ isochron for metagabbro samples collected from Baijiantan region gives an isochron age of $333 \mathrm{Ma}$ (lherzolite samples included for comparison). (c) Isochron plot of ${ }^{147} \mathrm{Sm} /{ }^{144} \mathrm{Nd} \mathrm{v} \cdot{ }^{143} \mathrm{Nd} /{ }^{144} \mathrm{Nd}$ for metagabbro samples collected from Baikouquan region. (d) Rb-Sr isochron for metagabbro samples (except for Bk13) collected from Baikouquan region gives an isochron age of $333 \mathrm{Ma}$. 


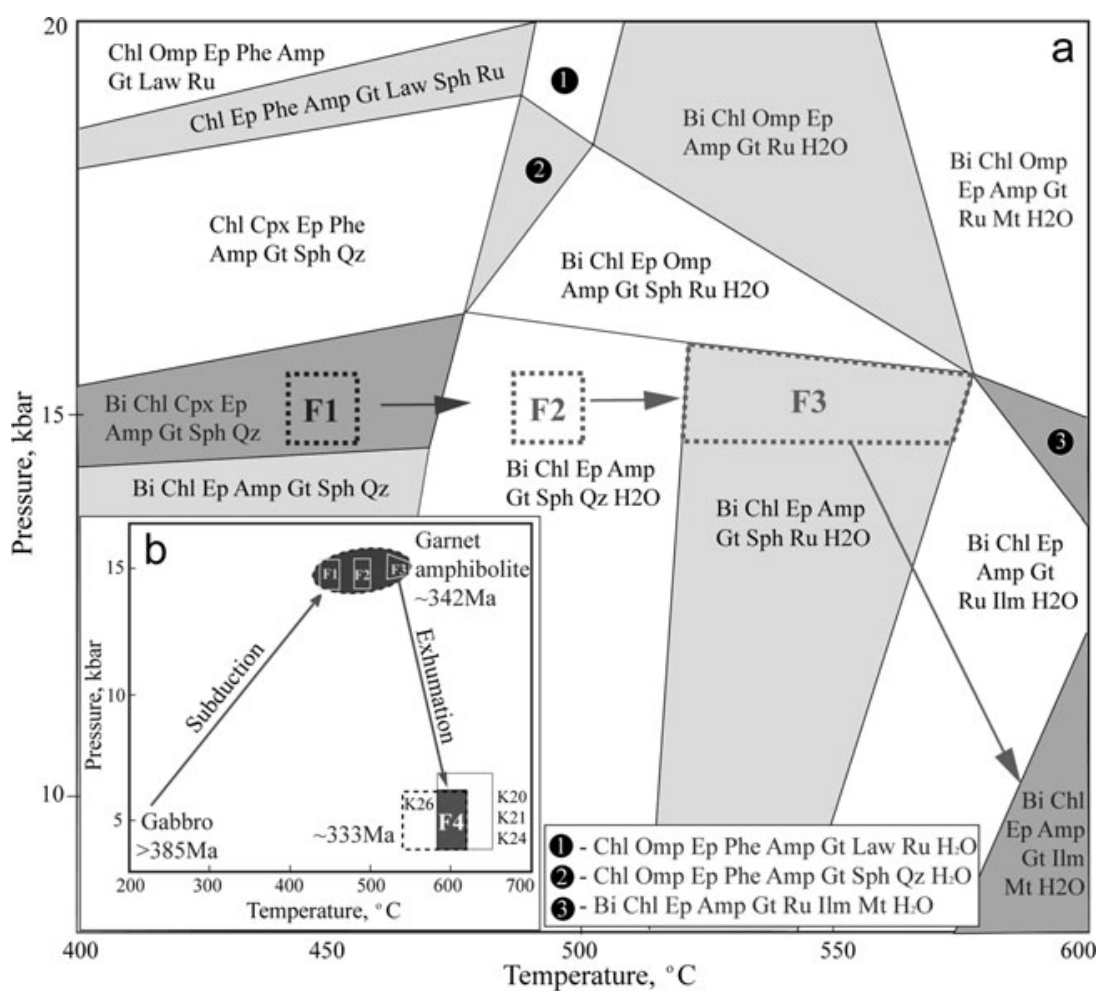

Figure 17. (a) Thermodynamic calculation results for a garnet amphibolite (sample K26) and (b) schematic $P-T$ path showing principal metamorphic stages recorded by the studied rock units and their possible relations. F1, F2 and F3 are equivalent to the $P-T$ environment in Figure 17a; the $P-T$ environment of F4 is calculated based on amphibole composition. $P-T$ path showing the evolution of gabbro (>385 Ma) from subduction (c. $342 \mathrm{Ma}$ ) to exhumation (c. $333 \mathrm{Ma}$ ). Mineral abbreviations: Amp - amphibole; Bi - biotite; Chl chlorite; Cpx - diopside-augite; Ep - epidote (zoisite included); Gt - garnet; Ilm - ilmenite; Law - lawsonite; Mt - magnetite; Omp omphacite; $\mathrm{Ru}$ - rutile; Qz - quartz; Sph - sphene.

(the TBB belt; Fig. 1c). This huge TBB ophiolitic belt is also similar in age to the TTKH ophiolitic belt. The Kujibai ophiolitic mélange in the TTKH, extending west to Kazakhstan and connecting with the Tarbahatai ophiolitic belt, was dated at 478 Ma by zircons separated from metagabbro (Zhu \& Xu, 2006).

Two Ordovician ophiolitic belts could therefore be identified in west Junggar: the TTKH belt located on the south boundary of the Chingiz-Tarbahatai arc, and the TBB belt located south of west Junggar (Fig. 1c). The Darbut-Sartohay ophiolitic belt, which is located between the TTKH and TBB Ordovician ophiolitic belts, was formed during the Early Devonian period. In such a tectonic scheme (Fig. 18) the TTKH was an accretionary terrane added to the south edge of the Chingiz-Tarbahatai arc then intruded by granitic rocks at $422-405 \mathrm{Ma}$ (Chen, Han \& Ji, 2010), and the TBB was an accretionary terrane amalgamated to the Junggar plate. The Darbut-Sartohay ophiolitic belt contains relics of the palaeo-oceanic floor.

Both the TTKH and TBB represent relics of Ordovician oceanic floor which subducted to the north under the Chingiz-Tarbahatai arc and to the south under the Junggar plate, respectively. Spreading of the Junggar oceanic floor probably did not stop until the Early Devonian period, as recorded by the Darbut ophiolitic belt located between the TTKH and TBB ophiolitic belts (Fig. 18a-c). The Kazakhstan-Junggar plate finally formed during the Early Devonian period, following closure of the Junggar ocean. The widespread early Carboniferous volcanic-sedimentary basins, filled by volcanic rocks, tuffaceous sandstone and siltstone intercalated with chert both in Chingiz-Tarbahatai and west Junggar, may have been part of this process.

\section{Conclusion}

The early Palaeozoic oceanic floor in west Junggar, represented by the Ordovician ophiolitic belts of TTKH and TBB, subducted to the north under the Chingiz-Tarbahatai arc and to the south under Junggar plate, respectively. The Baijiantan-Baikouquan ophiolite mélanges, representing the major part of the TBB, consist of serpentinized lherzolite, spinel-bearing serpentinite, metagabbro, garnet amphibolite, dolomite marble, pillow basalt and abyssal radiolarian chert. Metagabbro and garnet amphibolite show geochemical features of N-MORB with $\varepsilon_{\mathrm{Nd}(t)}>5.9$ and ${ }^{87} \mathrm{Sr}^{86} \mathrm{Sr}_{(t)}$ ratios of $0.7045-0.7054$. These rock units underwent metamorphism at different $P-T$ conditions; garnet amphibolite recorded a $P-T$ path starting from blueschist conditions, isobarically entering garnet amphibolite 


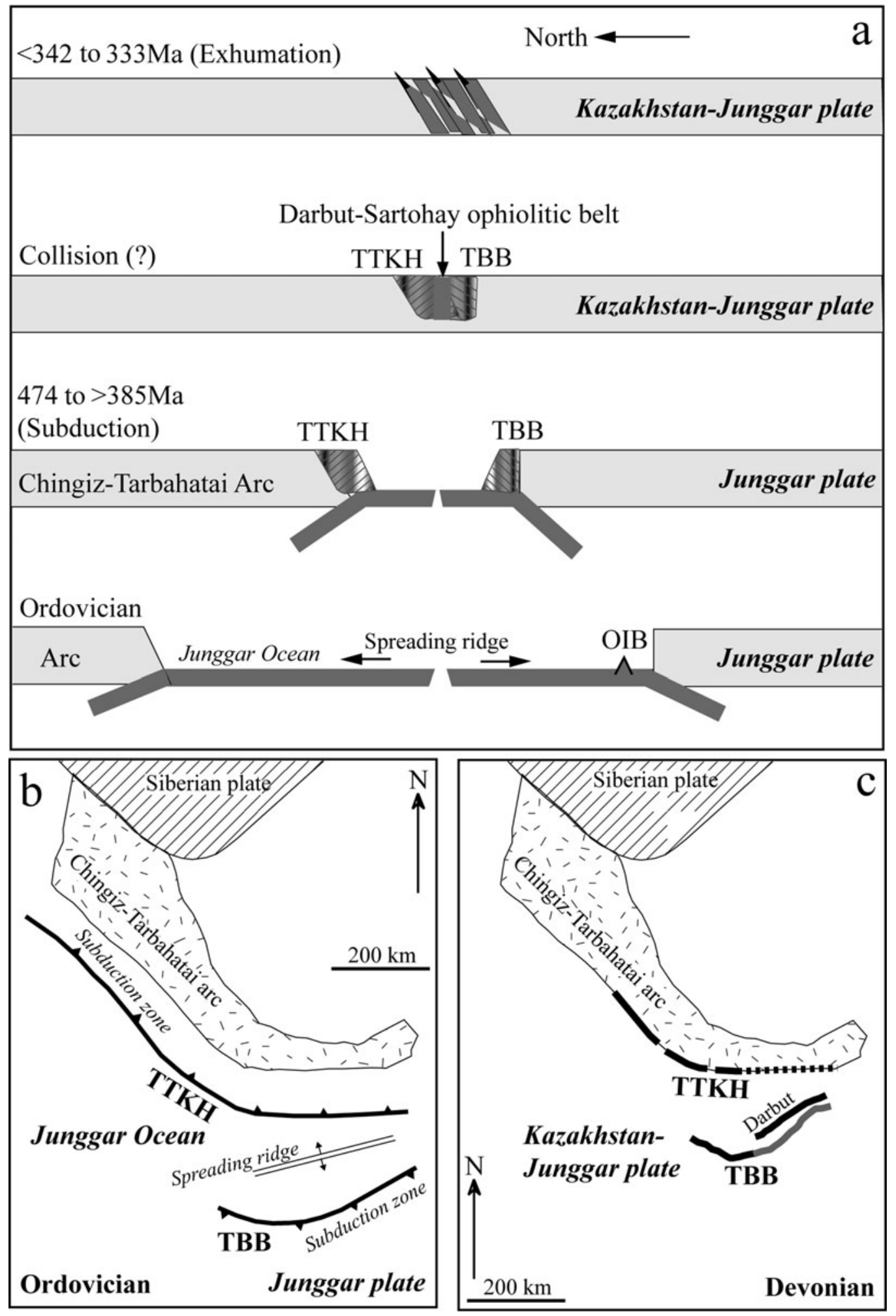

Figure 18. Tectonic evolution of west Junggar during Ordovician-Devonian time (see text for discussion).

conditions and finally passing through a $P-T$ environment of amphibolite stability based on thermodynamic calculations. The clockwise $P-T$ path suggests that the Baijiantan-Baikouquan ophiolitic mélanges were recycled via a subduction zone; the gabbro formed at $>385 \mathrm{Ma}$, was subducted, underwent metamorphism at $c .342 \mathrm{Ma}$ in the subduction zone and was finally exhumed at c. $333 \mathrm{Ma}$.

\section{Supplementary material}

To view supplementary material for this article, please visit http://dx.doi.org/10.1017/S0016756814000168

Acknowledgements. We would like to express our gratitude to Dr Réjean Hébert (Université Laval, Canada) and Professors Baofu Han and Guoqi He (Peking University) for their critical comments during this work. Professor Massonne H.-J. 
(Stuttgart University) introduced YZ to the PERPLE_X computer software package during a visit to Germany. Dr Phil Leat and two anonymous reviewers provided detailed critical comments and suggestions, which helped us to greatly improve this paper. Our gratitude is also extended to Dr Jeffrey Hedenquist and Phil Leat for correcting grammatical and syntax errors. Financial support for this study was provided by NSFC (Grant No. 41121062, 41372062, 41072041) and the International Science \& Technology Cooperation Program of China (Grant No. 2010DFB23390).

\section{References}

AN, F. \& ZHU, Y. F. 2009. SHRIMP U-Pb zircon ages of tuff in Baogutu formation and their geological significances. Acta Petrologica Sinica 25, 1437-45 (in Chinese with English abstract).

BAlCAEN, L., SchriJVer, I. D., Moens, L. \& VANHAECKe, F. 2005. Determination of the ${ }^{87} \mathrm{Sr} /{ }^{86} \mathrm{Sr}$ isotope ratio in USGS silicate reference materials by multi-collector ICP-mass spectrometry. International Journal of Mass Spectrometry 242, 251-5.

Chen, B. \& ZHU, Y. F. 2011. Petrology, geochemistry and zircon U-Pb chronology of gabbro in Darbut ophiolitic melange, Xinjiang. Acta Petrologica Sinica 27, 1746-58 (in Chinese with English abstract).

Chen, J. F., HAN, B. F. \& Ji, J. Q. 2010. Zircon U-Pb ages and tectonic implications of Paleozoic plutons in northern West Junggar, North Xinjiang, China. Lithos 115, 13752.

Coleman, R. G. 1965. Eclogites and eclogites: their difference and similarities. Bulletin of the Geological Society of America 76, 483-580.

Coleman, R. G. 1989. Continental growth of northwest China. Tectonics 8, 621-35.

ConNOLly, J. A. D. 2005. Computation of phase equilibria by linear programming: a tool for geodynamic modeling and its application to subduction zone decarbonation. Earth and Planetary Science Letters 236, 524-41.

FENG, Y. M. 1986. Genetic environments and original types of ophiolites in west Junggar. Bulletion of Xi'an Institute of Geology and Mineral Resources, Chinese Academy of Geological Science 13, 37-45 (in Chinese with English Abstract).

Gerya, T. V., Perchuk, L. L., Triboulet, C., Audren, C. \& SEZ'KO, A. I. 1997. Petrology of the Tumanshet Zonal Metamorphic Complex, Eastern Sayan. Petrology 5-6, 503-33.

GU, P. Y., LI, Y. J., ZhANG, B., TonG, L. L. \& WANG, J. N. 2009. LA-ICPMS zircon U-Pb dating of gabbro in the Darbut ophiolite, western Junngar, China. Acta Petrologica Sinica 26, 1364-72 (in Chinese with English abstract).

Han, B. F., He, G. Q., Wang, X. C. \& GuO, Z. J. 2011. Late Carboniferous collision between the Tarim and Kazakhstan-Yili terranes in the western segment of the South Tian Shan Orogen, Central Asia, and implications for the Northern Xinjiang, western China. Earth-Science Reviews 109, 74-93.

HE, G. Q., LI, M. S., JiA, J. D. \& ZHOU, H. 2001. A discussion on age and tectonic significance of ophiolite in eastern Junggar, Xinjiang. Acta Scientiarum Naturalium Universitatis Pekinensis 37, 852-8 (in Chinese with English abstract).

He, G. Q., LiU, J. B., Zhang, Y. Q. \& XU, X. 2007. Karamay ophioliic mélange formed during Early Paleozoic in western Junggar basin. Acta Petrologica Sinica 23, 1573-6 (in Chinese with English abstract).

Heinhorst, J., Lehmann, B., Ermolov, P., Serykh, V. \& ZHurutin, S. 2000. Paleozoic crustal growth and metallogeny of Central Asia: evidence from magmatichydrothermal ore systems of Central Kazakhstan. Tectonophysics 328, 69-87.

Holland, T. J. B. \& Powell, R. 1998. An internally consistent thermodynamic data set for phases of petrological interest. Journal of Metamorphic Geology 16, 30943.

Khain, E. V., Bibikova, E. V. \& SAlnikova, E. E. 2003. The Palaeo-Asian ocean in the Proterozoic and early Paleozoic: new geochronologic data and palaeotectonic reconstructions. Precambrian Research 122, 329-58.

Kwon, S. T., TILTON, C. R. \& COLEMAN, R. G. 1989. Isotopic investigations on the tectonic of the west Junggar region, Xinjiang, China. Tectonics 8, 719-27.

MASSONNE, H.-J. 2009. Hydration, dehydration, and melting of metamorphosed granitic and dioritic rocks at highand ultrahigh-pressure conditions. Earth and Planetary Science Letters 288, 244-54.

Mossakovsky, A. A., Ruzhentsev, S. V., SAmygin, S. G. \& Kheraskova, T. N. 1994. Central Asian Fold Belt: geodynamic evolution and formational history. Geotectonics 27, 445-74.

Sengor, A. M. C., NATAL'In, B. A. \& Burtman, V. S. 1993. Evolution of the Altaid tectonic collage and Paleozoic crustal growth in Eurasia. Nature 364, 299-307.

Sun, S. S. \& MCDONOUGH, W. F. 1989. Chemical and isotope systematics of oceanic basalts: implications for mantle composition and processes. In Magmatism in the Ocean Basins (eds D. Saunders \& M. J. Norry), pp. 313-45. Geological Society of London, Special Publication no. 42.

UpAdHyAY, D., Scherer, E. E. \& MEZGER, K. 2008. Fractionation and mixing of $\mathrm{Nd}$ isotopes during thermal ionization mass spectrometry: implications for high precision ${ }^{142} \mathrm{Nd} /{ }^{144} \mathrm{Nd}$ analyses. Journal of Analytical Atomic Spectrometry 23, 561-8.

Wilhem, C., Windley, B. F. \& Stamp, G. M. 2012. The Altaids of Central Asia: A tectonic and evolutionary innovative review. Earth-Science Reviews 113, 303-41.

WiLliamS, I. S. 1998. U-Th-Pb geochronology by ion microprobe. In Applications of Microanalytical Techniques to Understanding Mineralization Processes (eds M. A. McKibben, W. C. Shanks III \& W. I. Ridley), pp. 1-35. Reviews in Economic Geology 7.

Windley, B. F., AleXeIEV, D. \& XIAO, W. J. 2007. Tectonic models for accretion of the Central Asian Orogenic Belt. Journal of the Geological Society of London 164, 31-47.

XiaO, W. J., Han, C. M. \& YuAN, C. 2008. Middle Cambrian to Permian subduction-related accretionary orogenesis of Northern Xinjiang, NW China: implications for the tectonic evolution of central Asia. Journal of Asian Earth Sciences 32, 102-17.

XU, X., HE, G. Q. \& LI, H. Q. 2006. Basic characteristics of the Karamay ophiolitic mélange, Xinjiang, and its zircon SHRIMP dating. Geology in China 33, 470-5 (in Chinese with English abstract).

YAKUBCHUK, A. 2004. Architecture and mineral deposit settings of the Altaid orogenic collage: a revised model. Journal of Asian Earth Sciences 23, 761-79.

YANG, G. X., LI, Y. J., SANTOSH, M., YANG, B. K., ZHANG, B. \& TONG, L. 2013. Geochronology and geochemistry of basalts from the Karamay ophiolitic melange in West Junggar (NW China): implications for DevonianCarboniferous intra-oceanic accretionary tectonics of 
the southern Altaids. Geological Society of America Bulletin 125, 401-19.

ZHANG, C. \& HUANG, X. 1992. The ages and tectonic settings of ophiolites in west Junggar, Xinjiang. Geological Review 38, 509-24 (in Chinese).

Zhang, J. E., XIAO, W. J., Han, C. M., MaO, Q., AO, S. J., GuO, Q. Q. \& MA, C. 2011. A Devonian to Carboniferous intra-oceanic subduction system in Western Junggar, NW China. Lithos 125, 592-606.

ZHANG, L. F. $1997 .{ }^{40} \mathrm{Ar} /{ }^{39} \mathrm{Ar}$ age of blueschists in Tangbale, western Junggar, Xinjiang, and its significance. Chinese Science Bulletin 42, 2178-81 (in Chinese).

Zhang, L. F., AI, Y. L., LI, X. P., Rubatto, D. \& Song, B. 2007. Triassic collision of western Tianshan orogenic belt, China: evidence from SHRIMP U-Pb dating of zircon from HP/UHP eclogitic rocks. Lithos 96, 26680.

ZHENG, Y.-F., WU, Y. B., ZHAO, Z. F., ZHANG, S. B., XU, P. \& WU, F. Y. 2005. Metamorphic effect on zircon Lu-Hf and $\mathrm{U}-\mathrm{Pb}$ isotope systems in ultrahigh-pressure metagranite and metabasite. Earth and Planetary Science Letters 240, 378-400.

ZHU, Y. F. 2011. Zircon U-Pb and muscovite ${ }^{40} \mathrm{Ar} /{ }^{39} \mathrm{Ar}$ geochronology of the gold-bearing Tianger mylonitized granite, granite, Xinjiang, northwest China: implications for radiometric dating of mylonitized magmatic rocks. Ore Geology Reviews 40, 108-21.

Zhu, Y. F., AN, F., XU, C. Y., GUO, H., XiA, F., XiAO, F., ZhanG, F., LIN, C., QIU, T. \& WeI, S. 2013a. Geology and $\mathrm{Au}$-Cu Deposits in the Hatu and its Adjacent Region (Xinjiang): Evolution and Prospecting Model. Beijing: Geological Publishing House, 161 pp. (in Chinese with English abstract).

Zhu, Y. F., Chen, B., XU, X., QIU, T. \& An, F. 2013b. A new geological map of the western Junggar, north Xinjiang (NW China): implications for Paleoenvironmental reconstruction. Episodes 36, 205-20.
Zhu, Y. F., Guo, X., Song, B., Zhang, L. F. \& Gu, L. B. 2009. Petrology, Sr-Nd-Hf isotopic geochemistry and zircon chronology of the Late Paleozoic volcanic rocks in the southwestern Tianshan Mountains, Xinjiang, NW China. Journal of the Geological Society of London 166, 1085-99.

Zhu, Y. F., Sun, S. H., Gu, L. B., Ogasawara, Y., JiAng, N. \& HonMA, H. 2001. Permian volcanism in the Mongolian orogenic zone, northeast China: geochemistry, magma sources and petrogenesis. Geological Magazine 138, 101-15.

ZHU, Y. F. \& XU, X. 2006. The discovery of Early Ordovician ophiolite mélange in Tarbahatai Mts, Xinjiang, NW China. Acta Petrologica Sinica 22, 2833-42 (in Chinese with English abstract).

Zhu, Y. F., Xu, X., Chen, B. \& Xue, X. Y. 2008. Dolomite marble and garnet amphilolite in the ophiolitic mélange in western Junggar: relics of the Early Paleozoic oceanic crust and its deep subduction. Acta Petrologica Sinica 24, 2767-77 (in Chinese with English abstract).

ZHU, Y. F., XU, X. \& WEI, S. N. 2007. Geochemistry and tectonic significance of OIB-type pillow basalts in western Mts of Karamay city (western Junggar), NW China. Acta Petrologica Sinica 23, 1739-48 (in Chinese with English abstract).

Zhu, Y. F., YAn, Q. M., MA, H. D. \& LehmanN, B. 2011. Recent advances in geology and exploration in the Balkash-western Junggar region (Kazakhstan and Xinjiang, China): report on the 'International workshop on the large Balkash-western Junggar copper-gold province', Karamay, Xinjiang, China, 22-27 August, 2011. Episodes 34, 208-11.

Zonenshain, L. P., Kuzmin, M. I. \& Natapov, L. M. 1990. Geology of the USSR: A Plate-tectonic Synthesis. American Geophysical Union, Washington, Geodynamics Series no. 21. 